\title{
Alguns Modelos de Filas Paralelas que são mais Eficientes do que Fila Única
}

\author{
Willian de Souza Pereira
}

Orientadora: Profa. Dra. Maria Creusa Bretas Salles

Dissertação apresentada ao Instituto de Ciências Matemáticas e de Computação - ICMC-USP, como parte dos requisitos para obtenção do título de Mestre em Ciências - Área: Ciências de Computação e Matemática Computacional.

USP - São Carlos

Junho de 2000 
Dedico este trabalho aos meus pais José (in memorian) e Maria, aos meus irmãos, particularmente ao José Filho (in memorian)e ao meu grande amor, Isa, que me deram todo o apoio que um homem precisa. 


\section{Agradecimentos}

À minha orientadora, Maria Creusa Breta Salles, exemplo de incentivo e confiança.

Aos professores Josemar Rodrigues, Marinho Gomes de Andrade Filho e Reinaldo Morábito Neto, que marcaram minha trajétoria. Particularmente aos dois últimos, que deram valiosas sugestões no exame de qualificação.

Ao Marcos Roberto Teixeira Primo, amigo integral e diferencial. Como um irmão, foi baluarte das filas paralelas da vida.

Ao Silmar Barbosa, o Forrest Gump de meus calundus. À Ana Lucia da Silva, com quem vi que "quem quer passar o Bojador tem de ir além da dor".

À turma do curso de verão 1/98 (Leonilce, Lucimary e Vanderly); ao Moisés e aos funcionários do ICMC-USP.

Aos colegas da Escola Técnica Federal de Mato Grosso, especialmente os da Coordenação de Secretariado.

Finalmente, a CAPES - PICDT, que subvencionou este trabalho.

obs.: A probabilidade de esquecimento de pessoas relevantes é 0,999 ; porém neste estágio do trabalho a esperança de que meu sistema esteja em equilíbrio é de 0,01 a 0,1 . 


\section{RESUMO}

Filas paralelas são de crucial importância em supermercados, computação paralela, tráfego, etc. Usualmente, em filas paralelas, o critério de decisão para a questão "qual fila juntar-se" é juntar-se à fila mais curta, embora possa-se mostrar que em determinadas circunstâncias este não é o melhor critério. Com esta abordagem, são apresentados resultados conhecidos na literatura que são analisados conjuntamente. Primeiramente apresentamos um sistema de fila $M /(M / 1)^{2}$, duas filas paralelas com o critério de juntar-se à fila mais curta. São apresentados a distribuição de equilíbrio para o processo conjunto $\left\{N_{1}, N_{2}\right\}$ e o tempo médio de espera na fila. Apresentamos também um sistema $M /(G / 1)^{2}$, onde o serviço geral é especial, implicando que juntar-se à fila mais longa é mais benéfico. Finalmente são apresentados os sistemas de filas $G /\left(\left(M_{1}+M_{2}\right) / 1\right)^{2}$ e $M /((M+G) / 1)^{2}$, onde o cliente esperto utiliza o critério de esperar e juntar-se à fila que produz o primeiro fim de serviço e seu tempo médio de espera é ainda menor. Resultados numéricos comparativos são apresentados. 


\section{ABSTRACT}

Parallel queues have crucial importance in supermarkets, parallel computation, traffic, etc. Usually regarding to parallel queues the decision rule for the question "what queue to join" is joining to the shortest queue although in some circumstances this is not the best criterium. Following such broaching results reported by the literature are presented and analyzed as a whole. Firstly one presents a queueing system $M /(M / 1)^{2}$, two parallel queues following the decision rule of joining the shortest queue. One presents the equilibrium distribution for the joint process $\left\{N_{1}, N_{2}\right\}$ and the average delay time in queue. We also present a system $M /(G / 1)^{2}$ in which general service time is special and joining the longest queue is suitable. Finally one presents the queues system $G /\left(\left(M_{1}+M_{2}\right) / 1\right)^{2}$ and $M /((M+G) / 1)^{2}$, where the smart customer uses a strategy of waiting and joining the queue which generates the first end of service and its average delay time is quite shorter. Numerical comparative results are presented. 


\section{Sumário}

1 Introdução 1

1.1 Revisão Bibliográfica . . . . . . . . . . . . . . . . . . 2

1.2 Descrição do Trabalho . . . . . . . . . . . . . . . . 3

2 Resultados Gerais de Filas Paralelas 5

2.1 Introdução . . . . . . . . . . . . . . . . 5

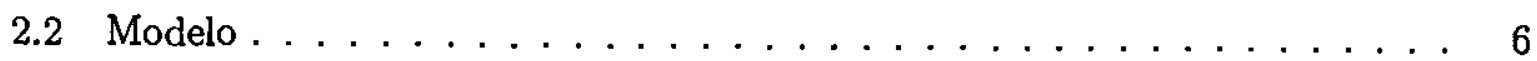

2.3 Análise do Modelo .................... 8

2.4 Distribuição do número de Clientes nas Filas 1 e 2 e no Sistema . . . . . 29

2.5 Resultados Numéricos . . . . . . . . . . . . . . . 31

2.6 Considerações Finais . . . . . . . . . . . . . . . . . 32

3 Filas Paralelas com Serviços Dicotômicos 33

3.1 Introdução . . . . . . . . . . . . . . . . . 33

3.2 Característica do Sistema Proposto . . . . . . . . . . . . . . 34

3.3 Resultados . . . . . . . . . . . . . . . . . . 35

3.4 Considerações Finais . . . . . . . . . . . . . . . . . . . . 42

4 Observando Filas Paralelas com Serviços Exponenciais 43

4.1 Introdução . . . . . . . . . . . . . . . . . . . . 43

4.2 Modelo........................... . . . 44

4.3 Análises das Estratégias . . . . . . . . . . . . . . . . . 45

4.4 Resultados Numéricos . . . . . . . . . . . . . . . 66

4.5 Considerações Finais . . . . . . . . . . . . . . . 68

5 Observando Filas Paralelas com Serviço Geral $\quad 69$

5.1 Introdução . . . . . . . . . . . . . . . . . 69 
5.2 Modelo............................ 70

5.3 Análise do Modelo com um Servidor com Tempo de Serviço Exponencial . 70

5.4 Análise do Modelo com Servidores Não-exponenciais . . . . . . . . . 83

5.5 Resultados Numéricos . . . . . . . . . . . . . . . . 91

5.6 Consideraçōes Finais . . . . . . . . . . . . . . . . 93

6 Comparação Numérica dos Resultados 95

6.1 Introdução . . . . . . . . . . . . . . . . . 95

6.2 Resultados Encontrados ..................... 96

7 Conclusōes Finais e Propostas Futuras 101

7.1 Proposta Futura . . . . . . . . . . . . . . . . . 102

$\begin{array}{ll}\text { A Algoritmo do Programa } & 103\end{array}$

$\begin{array}{ll}\text { Referências Bibliográficas } & 106\end{array}$ 


\section{Capítulo 1}

\section{Introdução}

No cotidiano, modelos de filas são de grande utilidade na análise e no projeto de sistemas de prestação de serviços. Os principais componentes de tais sistemas são os clientes, que chegam ao sistema à procura de serviços, e os servidores, que prestam serviço de atendimento. Sabemos exatamente o que são filas em decorrência das experiências que o dia-a-dia nos coloca. Certamente não é agradável entrar em uma fila e esperar pelo serviço, pois quando a espera é longa, ficamos aborrecidos. Se existem filas, passamos a comparar o desempenho da nossa fila com o das outras e, geralmente, somos levados a pensar como uma das leis de Murphy: "a fila que anda é a outra, mas não adianta trocar de fila pois a fila que anda é a outra".

Os problemas de filas tiveram basicamente sua origem no trabalho de Erlang, considerado o pai da Teoria de Filas, quando trabalhava em uma companhia telefônica estudando o problema de redimensionamento de centrais telefônicas. E, atualmente, a aplicação de teoria de fila se estende à análise de performance de sistemas de computação, comunicação, produção industrial, transportes etc.

Citaremos algumas aplicações dos modelos de filas que ocorrem freqüentemente: clientes num banco esperando por um atendimento; consumidores num supermercado esperando em frente aos caixas; aviões esperando para decolar (aterrissar) num aeroporto; chamadas telefônicas esperando serem roteadas pelo operador de PABX etc.

Este trabalho trata dos sistemas de filas em paralelo, onde descrevemos as caraterísticas de cada modelo posteriormente. Citamos o modelo de duas filas paralelas $A /(B / 1)^{2}$, onde 
$A$ refere-se ao processo de chegada e $B$, ao processo de serviço, sendo que $A$ (ou $B$ ) $=M$ : sistema markoviano, $A$ (ou $B$ ) $=G$, distribuição geral.

O propósito desta dissertação é demonstrar algumas medidas de desempenho dos sistemas de filas em paralelo, onde o fator dominante que as diferenciam é o critério usado pelo cliente ao decidir a qual fila se juntar.

\subsection{Revisão Bibliográfica}

Entre vários resultados encontrados na literatura, citaremos alguns sistemas de filas em paralelo.

Haight(57) foi o primeiro a considerar o problema de um sistema de filas em paralelo, onde o cliente que entra no sistema segue o critério tradicional de juntar-se à fila mais curta, com opção pela fila 1 quando as duas têm o mesmo comprimento. Ele calcula a distribuição de equilíbrio dó número de clientes nas filas 1 e 2 no sistema.

Whitt(86) mostra que juntar-se d̀ fila mais curta nem sempre é a melhor escolha. O modelo definido é um sistema $M /(G / 1)^{2}$, onde o tempo de serviço geral é especial, implicando que juntar-se d̀ fila mais longa do modelo é mais benéfico.

Hlynka et al.(94) e Hynka e Stanford(96) mostram que o cliente esperto, em um sistema de filas paralelas, espera e junta-se à fila que produz o primeiro fim de serviço, onde pode haver um tempo médio de espera menor.

Winston(77) estudou o desempenho do sistema de filas em paralelo $M /(M / 1)^{2}$. De fato, para esse modelo, com processo de chegada Poisson e tempos de serviços exponenciais, conhecido como modelo puramente markoviano, Winston(77) provou que o critério para unir-se d̀ fila mais curta é ótimo, com respeito a critérios mais fortes, baseados na ordenação estocástica (veja o livro do Wolff(89)).

Lehtonem(81) examinou o comportamento do processo de safdas do sistema de filas puramente markoviano, ou seja, sistemas em que os tempos de serviços e os tempos 
entre chegadas são representados por distribuições exponenciais. Desta forma concluiu que empregando as comparações de ordem estocástica para todo o processo de saída são, evidenternente, necessárias condições extras na distribuição do tempo de serviço. Contraexemplos são complexos de se construir, porque é difícil descrever o comportamento da política da distribuição do tempo de serviço - por exemplo, não é fácil de calcular o ternpo médio de espera no sistema em equilíbrio.

Flatto e Mckean(77) demonstram que o número esperado de clientes no sistema de filas $M /(M / 1)^{2}$ é maior do que no sistema de fila única. Un cliente que acaba de chegar ao sistema, segundo o processo markoviano, imediatamente observa o comprimento das filas e junta-se à mais curta; caso as filas apresentem o mesmo comprimento, escolhe aleatoriamente qualquer uma delas.

Kingman(61) estudou o sistema de filas $M /(M / 1)^{2}$, onde o processo de chegada é Poisson com média $2 \rho$, o tempo de serviço de cada servidor é exponencial com média unitária. Un cliente que acaba de chegar no sistema observa o comprimentos das filas é junta-se à mais curta; caso apresentem o mesmo comprimento, ele seleciona aleatoriamente qualquer uma delas. Ele mostra que no tráfico pesado com a taxa de ocupação igual a $\rho^{2}$, o número esperado de clientes no sistema de filas paralelas é igual ao de um sistema de fila única.

\subsection{Descrição do Trabalho}

Esta dissertação está constitứda do seguinte modo:

No capítulo 2, apresentaremos o modelo de Haight(57) que utiliza as equações globais de balanço para obtermos a distribuição de equilíbrio e a solução será obtida através da aplicação das funções geradoras de probabilidades bidimensionais.

No capítulo 3 será abordado o modelo de Whitt(86). Este mostra que o critério de juntar-se a uma fila mais curta nern sernpre é a melhor escolha. Indica como calcular o tempo médio de permanência no sistema. 
No capítulo 4, apresentaremos o modelo de Hlynka et al.(94) que estuda o problema de observar filas paralelas com serviços exponenciais. É calculado o tempo médio de permanência do cliente esperto no sistema, considerando o limite nas taxas de serviços.

No capítulo 5, apresentaremos o modelo Stanford e Hlynka(96) que estuda o problema de observar filas paralelas com serviço geral. É calculado o tempo médio de permanência do cliente esperto no sistema, que espera produzir o primeiro fim de serviço em uma das filas e seleciona a fila na qual ocorreu a conclusão.

No capítulo 6, apresentaremos algumas medidas do modelo $M / M / 2$ e compararemos os resultados numéricos dessas medidas com os modelos de filas em paralelo, proposto nesta dissertação.

No capítulo 7, apresentaremos as conclusões finais, bem como algumas propostas para futuras pesquisas. 


\section{Capítulo 2}

\section{Resultados Gerais de Filas Paralelas}

\subsection{Introdução}

Neste capítulo apresentamos um modelo de filas em paralelo, a partir do artigo de Haight(57), Two Queues in Parallel. Este modelo é um sistema de filas $M /(M / 1)^{2}$ no qual um cliente que chega ao sistema, segundo um processo Poisson, imediatamente observa o número de clientes presentes esperando e sendo servido em cada uma das filas e utiliza o critério de juntar-se à fila mais curta. Caso as filas apresentem o mesmo comprimento, o cliente seleciona a fila 1.

Apresentamos na Seção 2.2 o modelo e a notação do sistema de duas filas paralelas proposto no referido artigo.

Na Seção 2.3 calculamos as equações de equilíbrio do sistema. Para isso devemos desenvolver as funções geradoras de probabilidade bidimensionais e, a partir delas, obtemos a distribuição de equilíbrio conjunta, e as marginais do processo bidimensional que conta o número de clientes nas filas 1 e 2.

Na seção 2.4 apresentamos a distribuição de equilíbrio para o número total de clientes no sistema. Na Seção 2.5 apresentamos um resultado numérico e, finalmente, na Seção 2.6 , as consideraçōes finais. 


\subsection{Modelo}

Nesta seção descrevemos o sistema de filas $M(/ M / 1)^{2}$ que apresenta as seguintes características: dois servidores, cada um com sua própria fila; os dois servidores atendem os clientes com tempos de serviços distribuídos exponencialmente i.i.d. e com mesma taxa de atendimento; com disciplina First-Come, First Served (FCFS) - os clientes são servidos na ordem de chegada; os tempos entre as chegadas formam uma seqüência de variáveis aleatórias i.i.d. com distribuições exponenciais. Os tempos entre as chegadas e os tempos de serviço de cada um dos servidores são independentes.

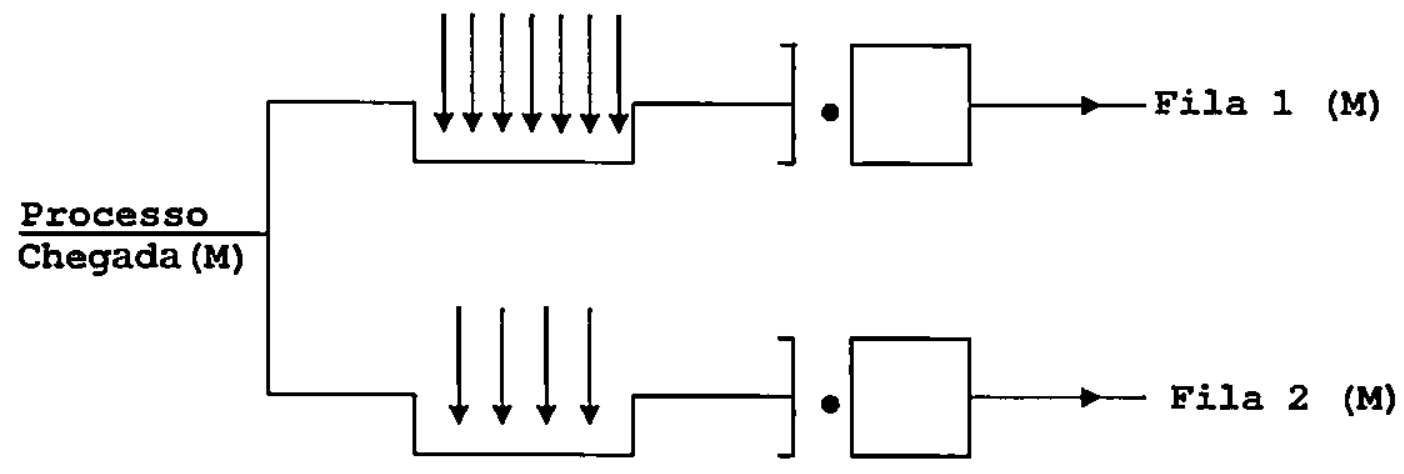

Figura 2.1: Uma representação do modelo $M /((M) / 1)^{2}$

O cliente que chega ao sistema observa antes de entrar os comprimentos das filas e toma uma decisão de acordo com um dos critérios abaixo:

- se ambas as filas apresentam o mesmo comprimento, o cliente junta-se à fila 1;

- se a fila 1 apresenta um comprimento menor, o cliente junta-se a ela;

- se a fila 1 apresenta um comprimento maior, o cliente deve unir-se à fila 2.

Sejam $X(t)$ e $X^{\prime}(t)$ os processos que contam o número de clientes nas filas 1 e 2 num instante $t ; X$ e $X^{\prime}$, os processos em equilíbrio; seja a seguinte notação: 
$p_{x y}(t):$ probabilidade conjunta do processo $X(t)$ e $X^{\prime}(t)$ num instante $t$, nos estados $x$ e $y$, respectivamente;

$p_{x y}$ : probabilidade conjunta do processo $X$ e $X^{\prime}$ em equilíbrio;

$p_{x .}(t) \quad$ : probabilidade marginal do processo $X(t)$ num instante $t$

$p_{x .} \quad$ : probabilidade marginal do processo $X$ em equilibrio;

$p_{. y}(t) \quad$ : probabilidade marginal do processo $X^{\prime}(t)$ num instante $t ;$

$p_{. y} \quad$ : probabilidade marginal do processo $X^{\prime}$ em equilíbrio;

$E[X] \quad$ : número médio de clientes na fila 1 em equilíbrio;

$E[X\rceil \quad$ : número médio de clientes na fila 2 em equilíbrio;

$\operatorname{Var}[X] \quad$ : variância do número de clientes na fila 1 em equilíbrio;

$\operatorname{Var}\left[X^{\prime}\right]$ : variância do número de clientes na fila 2 em equilíbrio.

Para o sistema em equilíbrio, as esperanças e as variâncias são dadas por:

(i) $E[X]=\sum_{x=0}^{\infty} x p_{x .}=m, E\left[X^{\prime}\right]=\sum_{y=0}^{\infty} y p_{. y}=m^{\prime}$;

(ii) $\operatorname{Var}[X]=\nu=\sigma^{2}, \operatorname{Var}\left[X^{\prime}\right]=\nu^{\prime}=\sigma^{\prime 2}$;

(iii) $m_{y}=E\left[X \mid X^{\prime}=y\right], \quad m_{x}^{\prime}=E\left[X^{\prime} \mid X=x\right]$,

$\nu_{y}=\operatorname{Var}\left[X \mid X^{\prime}=y\right], \quad \nu_{x}^{\prime}=\operatorname{Var}\left[X^{\prime} \mid X=x\right]$.

Desta notação podemos escrever os momentos da primeira e segunda ordem da seguinte forms:

1. $\sum_{y=0}^{\infty} y p_{x y}=m_{x}^{\prime} p_{x .}$ e $\sum_{x=0}^{\infty} x p_{x y}=m_{y} p_{. y}$

2. $\sum_{y=0}^{\infty} y^{2} p_{x y}=\left(\nu_{x}^{\prime}+m_{x}^{\prime 2}\right) p_{x .} . \quad$ e $\sum_{x=0}^{\infty} x^{2} p_{x y}=\left(\nu_{y}+m_{y}^{2}\right) p_{. y}$ 
Calculando as médias condicionadas, obtemos:

$$
\begin{aligned}
\sum_{y=0}^{\infty} y p_{x y} & =\sum_{y=0}^{\infty} y P\left[X=x, X^{\prime}=y\right]=\sum_{y=0}^{\infty} y P\left[X^{\prime}=y \mid X=x\right] P[X=x] \\
& =E\left[X^{\prime} \mid X=x\right] . P[X=x] \\
& =m_{x}^{\prime} p_{x .}
\end{aligned}
$$

e

$$
\begin{aligned}
\sum_{x=0}^{\infty} x p_{x y} & =\sum_{x=0}^{\infty} x P\left[X=x, X^{\prime}=y\right]=\sum_{x=0}^{\infty} x P\left[X=x \mid X^{\prime}=y\right] P\left[X^{\prime}=y\right] \\
& =E\left[X \mid X^{\prime}=y\right] . P\left[X^{\prime}=y\right] \\
& =m_{y} p_{. y .}
\end{aligned}
$$

Analogamente para os momentos condicionados de segunda ordem, obtemos:

$$
\begin{aligned}
\sum_{y=0}^{\infty} y^{2} p_{x y} & =\sum_{y=0}^{\infty} y^{2} P\left[X=x, X^{\prime}=y\right] \\
& =\sum_{y=0}^{\infty} y^{2} P\left[X^{\prime}=y \mid X=x\right] P[X=x]=E\left[X^{\prime 2} \mid X=x\right] P[X=x] \\
& =\left(V_{a r}\left[X^{\prime} \mid X=x\right]+\left(E\left[X^{\prime} \mid X=x\right]\right)^{2}\right) P[X=x] \\
& =\left(\nu_{x}^{\prime}+m^{\prime 2}\right) p_{x} .
\end{aligned}
$$

e

$$
\begin{aligned}
\sum_{x=0}^{\infty} x^{2} p_{x y} & =\sum_{x=0}^{\infty} x^{2} P\left[X=x, X^{\prime}=y\right]=\sum_{x=0}^{\infty} x^{2} P\left[X=x \mid X^{\prime}=y\right] P\left[X^{\prime}=y\right] \\
& =E\left[X^{2} \mid X^{\prime}=y\right] P\left[X^{\prime}=y\right] \\
& =\left(\operatorname{Var}\left[X \mid X^{\prime}=y\right]+\left(E\left[X \mid X^{\prime}=y\right]\right)^{2}\right) P\left[X^{\prime}=y\right] \\
& =\left(\nu_{y}+m_{y}^{2}\right) p_{. y} .
\end{aligned}
$$

\subsection{Análise do Modelo}

Nesta seção calculamos as equaçōes de balanço global do sistema, usando a técnica da função geratriz de probabilidade. Seja $p_{x y}(t)$ a probabilidade conjunta do processo $X(t)$ e $X^{\prime}(t)$, de haver $x, y$ clientes, respectivamente, nas filas 1 e 2 no sistema no instante $t$. Podemos analisar a evolução das filas durante um intervalo infinitesimal $\Delta t$. Suponhamos que existam $x, y$ clientes no sistema no instante $t+\Delta t$. Podemos dizer que a ocorrência desse evento é formada pela união dos seguintes eventos:

(i) haver $x, y$ clientes nas filas 1 e 2 no sistema no instante $t$, nāo haver chegado nenhum cliente no intervalo $\Delta t$, e não haver um fim de serviço nas filas 1 e 2 nesse intervalo (caso $\mathbf{A}$ ); 
(ii) haver $(x-1, y)$ ou $(x, y-1)$ clientes no sistema no instante $t$, ter chegado um cliente e entrado na fila 1 , para o estado $(x-1, y)$, ou ter entrado na fila 2 , para o estado $(x, y-1)$, e não haver nenhum fim de serviço nas filas 2 e 1 , respectivamente (casos B e C, respectivamente);

(iii) haver $(x+1, y)$ ou $(x, y+1)$ clientes no sistema no instante $t$, não haver chegado nenhum cliente no intervalo $\Delta t$, e haver um fim de serviço na fila 1 para o estado $(x+1, y)$ ou um fim de serviço na fila 2 para o estado $(x, y+1)$, nesse intervalo (caso D).

Dessa maneira, as três situações de transição não nulas indicadas acima são calculadas no primeiro passo a seguir. Enuciamos a seguir os passos para obtermos as equações globais do sistema:

a) considerando $p_{x y}(t+\Delta t)$ como a probabilidade conjunta do processo $X(t)$ e $X^{\prime}(t)$ estar no estado $(x, y)$ no instante $t+\Delta t$, obtém-se $\frac{d}{d t} p_{x y}(t), x, y=0,1,2 \ldots$;

b) assumindo equilfbrio quando $t \rightarrow \infty$, encontramos as equações de probabilidades conjuntas do sistema, fazendo $\lim _{t \rightarrow \infty} \frac{d}{d t} p_{x y}(t)=0$;

c) multiplicando-se por $s^{x}$ e $s^{\prime y}$, a $x, y$-ésima equação, respectivamente, e somando-se para $x, y$ todo o conjunto, obtém-se a função geratriz de probabilidade do sistema;

$$
\phi\left(s, s^{\prime}\right)=\sum_{x} \sum_{y} p_{x y} s^{x} s^{n}
$$

d) calculando a primeira e a segunda derivadas parciais do item anterior, obtemos as equações globais de balanço quando substituímos os valores de $s$ e $s^{\prime}$ por:
d1) $s=s^{\prime}=0$;
d2) $s=1, s=0$;
d3) $s=0, \quad s^{\prime}=1$;
d4) $s=s^{\prime}=1$.

Para facilitar a visualização e compreensão dos cálculos em cada caso, utilizamos o sinal + para separar o final dos cálculos do caso A e início do caso B, o sinal + para 
separar o final dos cálculos do caso B e início dos cálculos do caso C e o sinal + para separar os final dos cálculos do caso $\mathrm{C}$ e início dos cálculos do caso $\mathrm{D}$. Lembramos que as partes da função geradora de probabilidade nos quatro casos podem ser escritas da seguinte maneira

$$
A\left(s, s^{\prime}\right)+B\left(s, s^{\prime}\right)+C\left(s, s^{\prime}\right)+D\left(s, s^{\prime}\right)=0
$$

e suas derivadas parciais também somam zero, qualquer que seja o valor $\left(s, s^{\prime}\right)$.

Vamos definir as funções indicadoras $I(x, y)$ e $J(x, y)$ :

$$
\begin{gathered}
\text { Se } \quad x<y \rightarrow\left\{\begin{array}{l}
I(x, y)=1 \\
J(x, y)=0
\end{array}\right. \\
\text { Se } \quad x>y+1 \rightarrow\left\{\begin{array}{l}
I(x, y)=0 \\
J(x, y)=1
\end{array}\right. \\
\text { Se } x=y \text { ou } \quad x-1=y \rightarrow|x-y|=1\left\{\begin{array}{l}
I(x, y)=1 \\
J(x, y)=1
\end{array}\right.
\end{gathered}
$$

Vamos calcular o primeiro passo:

$$
\begin{gathered}
p_{x y}(t+\Delta t)=(1-\lambda \Delta t)(1-\mu \Delta t)\left(1-\mu^{\prime} \Delta t\right) p_{x y}(t)+(1-\lambda \Delta t)(1-\mu \Delta t) \mu^{\prime} \Delta t p_{x y}(t)+ \\
(1-\lambda \Delta t)\left(1-\mu^{\prime} \Delta t\right) \mu \Delta t p_{x+1, y}+ \\
(1-\mu \Delta t)\left(1-\mu^{\prime} \Delta t\right) \lambda \Delta t\left[I(x, y) p_{x-1, y}(t)+J(x, y) p_{x, y-1}(t)\right]= \\
=\left(1-\mu \Delta t-\lambda \Delta t+\lambda \mu \Delta t^{2}\right)\left(1-\mu^{\prime} \Delta t\right) p_{x y}(t)+(1-\mu \Delta t-\lambda \Delta t) \mu^{\prime} \Delta t p_{x, y+1}(t)+ \\
\left(1-\mu^{\prime} \Delta t-\lambda \Delta t\right) \mu \Delta t p_{(x+1) y}(t)+\left(1-\mu^{\prime} \Delta t-\mu \Delta t\right) \lambda \Delta t\left[I(x, y) p_{x-1, y}(t)+\right. \\
\left.J(x, y) p_{x, y-1}(t)\right]=
\end{gathered}
$$


Omitindo-se os termos de $o(\Delta t)$, temos

$$
\begin{aligned}
= & \left(1-\mu^{\prime} \Delta t-\mu \Delta t-\lambda \mu \Delta t\right) p_{x y}(t)+\mu^{\prime} \Delta t p_{x, y+1}(t)+\mu \Delta t p_{x+1, y}(t)+ \\
& \lambda \Delta t\left[I(x, y) p_{x-1, y}(t)+J(x, y) p_{x, y-1}(t)\right]= \\
= & p_{x y} \Delta t-\left(\lambda+\mu+\mu^{\prime}\right) \Delta t p_{x y}(t)+\mu^{\prime} \Delta t p_{x, y+1}(t)+\mu \Delta t p_{x+1, y}(t)+ \\
& \lambda \Delta t\left[I(x, y) p_{x-1, y}(t)+J(x, y) p_{x, y-1}(t)\right]
\end{aligned}
$$

Subtraindo-se o fator $p_{x y}(t) \Delta t$ em ambos os lados desta equação, dividindo-se por $\Delta t$, temos

$$
\begin{gathered}
\frac{p_{x y}(t+\Delta t)-p_{x y} \Delta t}{\Delta t}=\frac{-\left(\lambda+\mu+\mu^{\prime}\right) \Delta t p_{x y}(t)}{\Delta t}+\frac{\mu^{\prime} \Delta t p_{x, y+1}(t)}{\Delta t}+\frac{\mu \Delta t p_{x, y+1}(t)}{\Delta t}+ \\
\frac{\lambda \Delta t\left[I(x, y) p_{x-1, y}(t)+J(x, y) p_{x, y-1}(t)\right]}{\Delta t}
\end{gathered}
$$

Aplicando-se o limite para $\Delta t \rightarrow 0$, temos

$$
\begin{gathered}
\lim _{\Delta t \rightarrow 0} \frac{p_{x y}(t+\Delta t)-p_{x y} \Delta t}{\Delta t}=\lim _{\Delta t \rightarrow 0} \frac{-\left(\lambda+\mu+\mu^{\prime}\right) \Delta t p_{x y}(t)}{\Delta t}+\lim _{\Delta t \rightarrow 0} \frac{\mu^{\prime} \Delta t p_{x, y+1}(t)}{\Delta t}+ \\
\lim _{\Delta t \rightarrow 0} \frac{\mu \Delta t p_{x(y+1)}(t)}{\Delta t}+\lim _{\Delta t \rightarrow 0} \frac{\lambda \Delta t\left[I(x, y) p_{x-1, y}(t)+J(x, y) p_{x, y-1}(t)\right]}{\Delta t}= \\
p_{x y}^{\prime}(t)=-\left(\lambda+\mu+\mu^{\prime}\right) p_{x y}(t)+\mu^{\prime} p_{x(y+1)}(t)+\mu p_{x(y+1)}(t)+\lambda\left[I(x, y) p_{x-1, y}(t)+J(x, y) p_{x, y-1}(t)\right]
\end{gathered}
$$

Calculemos agora o segundo passo. Assumindo-se o equilíbrio quando $t \rightarrow \infty$, então, da equação (1.1), temos

$$
-\left(\lambda+\mu+\mu^{\prime}\right) p_{x y}+\mu^{\prime} p_{x(y+1)}+\mu p_{x(y+1)}+\lambda\left[I(x, y) p_{x-1, y}+J(x, y) p_{x, y-1}\right]=0
$$

Calculemos o terceiro passo. Multiplicando-se por $x, y$-ésimo membro da equação (1.2) por $s^{x} s^{\prime y}$, e somando-se para todo $x$ e $y$, temos

$$
\sum_{x=0}^{\infty} \sum_{y=0}^{\infty}-\left(\lambda+\mu+\mu^{\prime}\right) p_{x y} s^{x} s^{\prime y}+\sum_{x=0}^{\infty} \sum_{y=0}^{\infty} \mu^{\prime} p_{x(y+1)} s^{x} s^{\prime y}+\sum_{x=0}^{\infty} \sum_{y=0}^{\infty} \mu p_{(x+1) y} s^{x} s^{\prime y}+
$$




$$
\begin{gathered}
\sum_{x=0}^{\infty} \sum_{y=0}^{\infty} \lambda\left[I(x, y) p_{x-1, y}+J(x, y) p_{x, y-1}\right] s^{x} s^{\prime y}=0 \\
-\left(\lambda+\mu+\mu^{\prime}\right) \sum_{x=0}^{\infty} \sum_{y=0}^{\infty} p_{x y} s^{x} s^{\prime y}+\mu^{\prime} \sum_{x=0}^{\infty} \sum_{y=0}^{\infty} p_{x(y+1)} s^{x} s^{\prime y}+\mu \sum_{x=0}^{\infty} \sum_{y=0}^{\infty} p_{(x+1) y} s^{x} s^{\prime y}+ \\
\lambda \sum_{x=0}^{\infty} \sum_{y=0}^{\infty}\left[I(x, y) p_{x-1, y}+J(x, y) p_{x, y-1}\right] s^{x} s^{\prime y}=0
\end{gathered}
$$

Vamos analisar as expressōes de cada caso acima. No primeiro caso temos

$$
\begin{gathered}
A\left(s, s^{\prime}\right)=-\left(\lambda+\mu+\mu^{\prime}\right) \sum_{x=0}^{\infty} \sum_{y=0}^{\infty} p_{x y} s^{x} s^{\prime y}= \\
=-\lambda \sum_{x=0}^{\infty} \sum_{y=0}^{\infty} p_{x y} s^{x} s^{\prime y}-\mu \sum_{x=0}^{\infty} \sum_{y=0}^{\infty} p_{x y} s^{x} s^{\prime y}-\mu^{\prime} \sum_{x=0}^{\infty} \sum_{y=0}^{\infty} p_{x y} s^{x} s^{\prime y} .
\end{gathered}
$$

Quando substitúmos $x=0$ no segundo termo do segundo membro da equação (2.3) temos a ocorrência de transiçōes impossíveis, isto é, fim de serviço na fila 1 quando a mesma está vazia. Da mesma forma, quando substituímos $y=0$ no terceiro termo, ocorrem transiçōes impossíveis; isto é, fim de serviço na fila 2, quando a mesma encontra-se vazia; excluindo-se todas essas transições impossíveis, temos

$$
A\left(s, s^{\prime}\right)=-\left(\lambda+\mu+\mu^{\prime}\right) \sum_{x=0}^{\infty} \sum_{y=0}^{\infty} p_{x y} s^{x} s^{\prime y}+\mu \sum_{y=0}^{\infty} p_{0 y} s^{y y}+\mu^{\prime} \sum_{x=0}^{\infty} p_{x 0} s^{x}
$$

Desenvolvendo-se o segundo membro desta equação temos

$$
\begin{gathered}
A\left(s, s^{\prime}\right)=-\left(\lambda+\mu+\mu^{\prime}\right) \sum_{x=0}^{\infty}\left[p_{x 0} s^{x}+p_{x 1} s^{x} s^{\prime}+p_{x 2} s^{x} s^{\prime 2}+p_{x 3} s^{x} s^{\prime 3}+\ldots\right]+\mu\left[p_{00}+p_{01} s^{\prime}+\right. \\
\left.p_{02} s^{\prime 2}+p_{03} s^{\prime 3}+p_{04} s^{4}+\ldots\right]+\mu^{\prime}\left[p_{00}+p_{10} s+p_{20} s^{2}+p_{30} s^{3}+\ldots\right]= \\
=-\left(\lambda+\mu+\mu^{\prime}\right)\left[p_{00}+p_{10} s+p_{20} s^{2}+\ldots+p_{01} s^{\prime}+p_{11} s s^{\prime}+p_{21} s^{2} s^{\prime}+\ldots+p_{02} s^{\prime 2}+p_{12} s s^{\prime 2}+\right. \\
\left.p_{22} s^{2} s^{\prime 2}+\ldots+p_{03} s^{\prime 3}+p_{13} s s^{\prime 3}+p_{23} s^{2} s^{\prime 3}+\ldots\right]+\mu\left[p_{00}+p_{01} s^{\prime}+p_{02} s^{\prime 2}+p_{03} s^{\prime 3}+\right. \\
\left.p_{04} s^{\prime 4}+\ldots\right]+\mu^{\prime}\left[p_{00}+p_{10} s+p_{20} s^{2}+p_{30} s^{3}+\ldots\right]
\end{gathered}
$$


No segundo caso temos

$$
\begin{aligned}
B\left(s, s^{\prime}\right) & =\mu^{\prime} \sum_{x=0}^{\infty} \sum_{y=0}^{\infty} p_{x(y+1)} s^{x} s^{\prime y}=\frac{\mu^{\prime}}{s^{\prime}} \sum_{x=0}^{\infty} \sum_{y=0}^{\infty} p_{x(y+1)} s^{x} s^{\prime y+1}= \\
& =\frac{\mu^{\prime}}{s^{\prime}} \sum_{x=0}^{\infty}\left[p_{x 1} s^{x} s^{\prime 1}+p_{x 2} s^{x} s^{\prime 2}+\ldots+p_{x 0} s^{x}-p_{x 0} s^{x}\right]= \\
& =\frac{\mu^{\prime}}{s^{\prime}} \sum_{x=0}^{\infty} \sum_{y=0}^{\infty} p_{x y} s^{x} s^{\prime y}-\frac{\mu^{\prime}}{s^{\prime}} \sum_{x=0}^{\infty} p_{x 0} s^{x}=
\end{aligned}
$$

Logo,

$$
\begin{array}{r}
B\left(s, s^{\prime}\right)=\mu^{\prime}\left[p_{01}+p_{11} s+p_{21} s^{2}+p_{31} s^{3}+p_{41} s^{4}+\ldots+p_{02} s^{\prime}+p_{12} s s^{\prime}+p_{22} s^{2} s^{\prime}+p_{32} s^{3} s^{\prime}+\ldots+\right. \\
\left.p_{03} s^{\prime 2}+p_{13} s s^{\prime 2}+p_{23} s^{2} s^{\prime 2}+p_{33} s^{3} s^{\prime 2}+\ldots+p_{04} s^{3}+p_{14} s s^{\prime 3}+p_{24} s^{2} s^{\prime 3}+\ldots\right]
\end{array}
$$

Observamos que o termo $\frac{\mu^{\prime}}{s^{\prime}} \sum_{x=0}^{\infty} p_{x 0} s^{x}$ representa todas as transições impossiveis de ter um fim de serviço na fila 2 quando a mesma está vazia, devemos excluir todas estas transiçōes.

No terceiro caso temos

$$
\begin{aligned}
C\left(s, s^{\prime}\right) & =\mu^{\prime} \sum_{x=0}^{\infty} \sum_{y=0}^{\infty} p_{x+1, y} s^{x} s^{\prime y}=\frac{\mu}{s} \sum_{x=0}^{\infty} \sum_{y=0}^{\infty} p_{x+1, y} s^{x+1} s^{\prime y}= \\
& =\frac{\mu}{s} \sum_{y=0}^{\infty}\left[p_{1, y} s^{1} s^{\prime y}+p_{2, y} s^{2} s^{\prime y}+\ldots+p_{0, y} s^{y}-p_{0, y} s^{\prime y}\right]= \\
& =\frac{\mu}{s} \sum_{x=0}^{\infty} \sum_{y=0}^{\infty} p_{x y} s^{x} s^{\prime y}-\frac{\mu}{s} \sum_{y=0}^{\infty} p_{0 y} s^{\prime y}=
\end{aligned}
$$

Desenvolvendo-se o segundo termo desta equação temos

$$
\begin{gathered}
C\left(s, s^{\prime}\right)=\mu\left[p_{10}+p_{11} s^{\prime}+p_{12} s^{\prime^{2}}+p_{13}{s^{\prime 3}}^{3}+\ldots+p_{20} s+p_{21} s s^{\prime}+p_{22} s s^{\prime 2}+p_{23} s s^{\prime 3}+\ldots+\right. \\
\left.p_{30} s^{2}+p_{31} s^{2} s^{\prime}+p_{32} s^{2} s^{\prime 2}+\ldots\right]
\end{gathered}
$$

Observamos que o termo $\frac{\mu}{s} \sum_{y=0}^{\infty} p_{0 y} s^{\prime y}$ representa todas as transiçōes impossiveis de ter 
um fim de serviço na fila 1 quando a mesma está vazia, devemos excluir todas estas transições.

No quarto caso temos

$$
\begin{aligned}
& D\left(s, s^{\prime}\right)=\lambda \sum_{x=0}^{\infty} \sum_{y=0}^{\infty}\left[I(x, y) p_{x-1, y}+J(x, y) p_{x, y-1}\right] s^{x} s^{\prime y}= \\
& =\lambda\left[\sum_{x=0}^{\infty} \sum_{y=0}^{\infty} I(x, y) p_{x-1, y} s^{x} s^{\prime y}+\sum_{x=0}^{\infty} \sum_{y=0}^{\infty} J(x, y) p_{x, y-1} s^{x} s^{\prime y}\right]= \\
& =\lambda\left[s \sum_{x=0}^{\infty} \sum_{y=0}^{\infty} I(x, y) p_{x-1, y} s^{x-1} s^{\prime y}+\sum_{x=0}^{\infty} \sum_{y=0}^{\infty} J(x, y) p_{x, y-1} s^{x} s^{y-1}\right]= \\
& =\lambda\left[s \sum_{y=0}^{\infty} I(1, y) p_{0 y} s^{\prime y}+s \sum_{y=0}^{\infty} I(2, y) p_{1 y} s^{1} s^{\prime y}+s \sum_{y=0}^{\infty} I(3, y) p_{1 y} s^{2} s^{\prime y}+\ldots+s^{\prime} \sum_{x=0}^{\infty} J(x, 1) p_{x, 0} s^{x}+\right. \\
& \left.s^{\prime} \sum_{x=0}^{\infty} J(x, 2) p_{x, 1} s^{x} s^{1}+s^{\prime} \sum_{x=0}^{\infty} J(x, 3) p_{x, 2} s^{x} s^{2}+\ldots\right]= \\
& =\lambda\left[s \sum_{y=0}^{\infty} I(1, y) p_{0 y} s^{\prime y}+s^{2} \sum_{y=0}^{\infty} I(2, y) p_{1 y} s^{y}+s^{3} \sum_{y=0}^{\infty} I(3, y) p_{1 y} s^{\prime y}+\ldots+\right. \\
& \left.s^{\prime} \sum_{x=0}^{\infty} J(x, 1) p_{x, 0} s^{x}+{s^{\prime 2}}^{2} \sum_{x=0}^{\infty} J(x, 2) p_{x, 1} s^{x}+s^{\prime 3} \sum_{x=0}^{\infty} J(x, 3) p_{x, 2} s^{x}+\ldots\right] \\
& =\lambda\left[s \sum_{y=0}^{\infty} I(1, y) p_{0 y} s^{\prime y}+s^{2} I(2,0) p_{10} s^{\prime 0}+s^{2} \sum_{y=1}^{\infty} I(2, y) p_{1 y} s^{\prime y}+s^{3} I(3,0) p_{20} s^{\prime 0}+\right. \\
& \left.s^{3} I(3,1) p_{21} s^{\prime 1}+s^{3} \sum_{y=2}^{\infty} I(3, y) p_{20} s^{0}+\ldots\right]+\lambda\left[s^{\prime} J(0,1) p_{0,0} s^{0}+s^{\prime} \sum_{x=1}^{\infty} J(x, 1) p_{x, 0} s^{x}+\right. \\
& s^{\prime 2} J(0,2) p_{0,1} s^{0}+s^{\prime 2} J(1,2) p_{1,1} s^{1}+s^{\prime 2} \sum_{x=2}^{\infty} J(x, 2) p_{x, 1} s^{x}+s^{\prime 3} J(0,3) p_{0,2} s^{0}+ \\
& \left.s^{\prime 3} J(1,3) p_{1,2} s^{1}+s^{\prime 3} J(2,3) p_{2,2} s^{2}+s^{\prime 3} \sum_{x=3}^{\infty} J(x, 3) p_{x, 2} s^{x}+\ldots\right]=
\end{aligned}
$$




$$
\begin{aligned}
D\left(s, s^{\prime}\right)= & \lambda\left[s \sum_{y=0}^{\infty} 1 p_{0 y} s^{\prime y}+s^{2} 0 p_{10} s^{\prime 0}+s^{2} \sum_{y=1}^{\infty} 1 p_{1 y} s^{\prime y}+s^{3} 0 p_{20} s^{\prime 0}+s^{3} 0 p_{21} s^{1}+s^{3} \sum_{y=2}^{\infty} 1 p_{20} s^{\prime 0}\right. \\
& +\ldots]+\lambda\left[s^{\prime} 0 p_{0,0} s^{0}+s^{\prime} \sum_{x=1}^{\infty} 1 p_{x, 0} s^{x}+s^{\prime 2} 0 p_{0,1} s^{0}+s^{\prime 2} 0 p_{1,1} s^{1}+s^{\prime 2} \sum_{x=2}^{\infty} 1 p_{x, 1} s^{x}+\right. \\
& \left.s^{\prime 3} 0 p_{0,2} s^{0}+s^{\prime 3} 0 p_{1,2} s^{1}+s^{3} 0 p_{2,2} s^{2}+s^{\prime 3} \sum_{x=3}^{\infty} 1 p_{x, 2} s^{x}+\ldots\right]= \\
= & \lambda\left[s \sum_{y=0}^{\infty} p_{0 y} s^{\prime y}+s^{2} \sum_{y=1}^{\infty} p_{1 y} s^{\prime y}+s^{3} \sum_{y=2}^{\infty} p_{2 y} s^{\prime y}+\ldots\right]+\lambda\left[s s^{\prime} \sum_{y=0}^{0} p_{1, y} s^{y}+s^{2} s^{\prime} \sum_{y=0}^{1} p_{2, y} s^{y}+\right. \\
& \left.s^{3} s^{\prime} \sum_{y=0}^{2} 1 p_{3, y} s^{\prime \nu}+\ldots\right]= \\
= & \lambda\left[s \sum_{y=0}^{\infty} p_{0 y} s^{0} s^{\prime y}+s \sum_{y=1}^{\infty} p_{1 y} s s^{\prime y}+s \sum_{y=2}^{\infty} p_{2 y} s^{2} s^{\prime y}+\ldots\right]+\lambda\left[s s^{\prime} \sum_{y=0}^{0} p_{1, y} s^{\prime y}+s^{2} s^{\prime} \sum_{y=0}^{1} p_{2, y} s^{\prime y}+\right. \\
& \left.s^{3} s^{\prime} \sum_{y=0}^{2} 1 p_{3, y} s^{\prime y}+\ldots\right] \\
= & \lambda s \sum_{x \leq y}^{\infty} p_{x y} s^{x} s^{\prime y}+\lambda s^{\prime} \sum_{x>y}^{\infty} p_{x y} s^{x} s^{\prime y}=
\end{aligned}
$$

Desenvolvendo-se os termos da equação acima temos

$$
\begin{gathered}
D\left(s, s^{\prime}\right)=\lambda\left[s\left(p_{00}+p_{01} s^{\prime}+p_{02} s^{\prime 2}+p_{03} s^{\prime 3}+\ldots\right)+s^{2}\left(p_{11} s^{\prime}+p_{12} s^{\prime 2}+p_{13} s^{3}+p_{14} s^{\prime 4}+\ldots\right)+\right. \\
\left.s^{3}\left(p_{22} s^{\prime 2}+p_{23} s^{3}+p_{24} s^{\prime 4}+\ldots\right)+\ldots\right]+\lambda\left[s s^{\prime} p_{10}+s^{2} s^{\prime}\left(p_{20}+p_{21} s^{\prime}\right)+s^{3} s^{\prime}\left(p_{30}+\right.\right. \\
\left.\left.p_{31} s^{\prime}+p_{32} s^{\prime 2}\right)+\ldots\right]
\end{gathered}
$$

Fazendo-se

$$
\phi\left(s, s^{\prime}\right)=\sum_{x=0}^{\infty} \sum_{y=0}^{\infty} p_{x y} s^{x} s^{\prime y} \quad \psi\left(s, s^{\prime}\right)=\sum_{x \leq y}^{\infty} p_{x y} s^{x} s^{\prime y}
$$

Logo,

$$
\begin{gathered}
D\left(s, s^{\prime}\right)=\lambda s \psi\left(s, s^{\prime}\right)+\lambda s^{\prime}\left[\phi\left(s, s^{\prime}\right)-\psi\left(s, s^{\prime}\right)\right]= \\
=\lambda s \psi\left(s, s^{\prime}\right)+\lambda s^{\prime}\left(\phi\left(s, s^{\prime}\right)-\psi\left(s, s^{\prime}\right)\right)
\end{gathered}
$$


Vamos calcular o quarto passo. A equação da função geratriz de probabilidade do sistema é dada pelo soma das equações (2.4), (2.5), (2.6) e (2.7). Temos

$$
\begin{aligned}
& -\left(\lambda+\mu+\mu^{\prime}\right)\left[p_{00}+p_{10} s+p_{20} s^{2}+\ldots+p_{01} s^{\prime}+p_{11} s s^{\prime}+p_{21} s^{2} s^{\prime}+\ldots+p_{02} s^{\prime 2}+p_{12} s s^{\prime 2}+\right. \\
& \left.p_{22} s^{2} s^{\prime 2}+\ldots+p_{03} s^{3}+p_{13} s s^{\prime 3}+p_{23} s^{2} s^{\prime 3}+\ldots\right]+\mu\left[p_{00}+p_{01} s^{\prime}+p_{02} s^{\prime 2}+p_{03} s^{\prime 3}+p_{04} s^{\prime 4}+\right. \\
& \ldots]+\mu^{\prime}\left[p_{00}+p_{10} s+p_{20} s^{2}+p_{30} s^{3}+\ldots\right]+\mu^{\prime}\left[p_{01}+s p_{11}+s^{2} p_{21}+s^{3} p_{31}+s^{4} p_{41}+\ldots+s^{\prime} p_{02}+\right. \\
& \left.s s^{\prime} p_{12}+s^{2} s^{\prime} p_{22}+s^{3} s^{\prime} p_{32}+\ldots+s^{\prime 2} p_{03}+s^{\prime 2} p_{13}+s^{2} s^{\prime 2} p_{23}+s^{3} s^{\prime 2} p_{33}+\ldots\right]+\mu\left[p_{10}+p_{11} s^{\prime}\right. \\
& \left.p_{12} s^{2}+\ldots+p_{20} s+p_{21} s s^{\prime}+p_{22} s s^{\prime 2}+p_{23} s s^{3}+\ldots+p_{30} s^{2}+p_{31} s^{2} s^{\prime}+p_{32} s^{2} s^{\prime 2}+\ldots\right]+ \\
& \lambda\left[s\left(p_{00}+p_{01} s^{\prime}+p_{02} s^{\prime 2}+p_{03} s^{\prime 3}+\ldots\right)+s^{2}\left(p_{11} s^{\prime}+p_{12} s^{\prime 2}+p_{13} s^{\prime 3}+p_{14} s^{4}+\ldots\right)+s^{3}\left(p_{22} s^{\prime 2}+\right.\right. \\
& \left.\left.p_{23} s^{\prime 3}+p_{24} s^{4}+\ldots\right)+\ldots\right]+\lambda\left[s s^{\prime} p_{10}+s^{2} s^{\prime}\left(p_{20}+p_{21} s^{\prime}\right)+s^{3} s^{\prime}\left(p_{30}+p_{31} s^{\prime}+p_{32} s^{\prime 2}\right)+\ldots\right]=0
\end{aligned}
$$

Substituindo-se $s=s^{\prime}=0$ na equação (2.9) temos

$$
-\left(\lambda+\mu+\mu^{\prime}\right) p_{00}+\mu p_{00}+\mu^{\prime} p_{00}+\mu p_{10}=0,
$$

logo,

$$
-\lambda \mathbf{p}_{00}+\mu \mathbf{p}_{10}+\mu^{\prime} \mathbf{p}_{10}=0
$$

Para $s=1$ e $s^{\prime}=0$ na equação (2.9) temos

$$
\begin{aligned}
& -\left(\lambda+\mu+\mu^{\prime}\right)\left(p_{00}+p_{10}+p_{20}+\ldots\right)+\mu p_{00}+\mu^{\prime}\left(p_{00}+p_{10}+p_{20}+\ldots\right)+\mu^{\prime}\left(p_{01}+p_{11}+p_{21}+\ldots\right)+ \\
& \mu\left(p_{10}+p_{20}+p_{30}+\ldots\right)+\lambda p_{00}=0 .
\end{aligned}
$$

Fazendo-se

$$
\begin{aligned}
& p^{\prime}=p_{.0}=\sum_{x=0}^{\infty} p_{x 0}=p_{00}+p_{10}+p_{20}+p_{30}+\ldots, \\
& p_{.1}=\sum_{x=0}^{\infty} p_{x 1}=p_{01}+p_{11}+p_{21}+\ldots
\end{aligned}
$$

temos

$$
-\lambda \mathbf{p}^{\prime}+\mu^{\prime} \mathbf{p}_{.1}+\lambda \mathbf{p}_{00}=\mathbf{0} .
$$


Para $s=0$ e $s^{\prime}=1$ na equação (2.9) temos

$$
\begin{aligned}
& -\left(\lambda+\mu+\mu^{\prime}\right)\left(p_{00}+p_{01}+p_{02}+\ldots\right)+\mu\left(p_{00}+p_{01}+p_{02}+\ldots\right)+\mu^{\prime} p_{00}+\mu^{\prime}\left(p_{01}+p_{02}+p_{03} \ldots\right)+ \\
& \mu\left(p_{10}+p_{11}+p_{12}+\ldots\right)=0 .
\end{aligned}
$$

Fazendo-se

$$
\begin{aligned}
& p=p_{0 .}=\sum_{y=0}^{\infty} p_{0 y}=p_{00}+p_{01}+p_{02}+p_{03}+\ldots, \\
& p_{1 .}=\sum_{y=0}^{\infty} p_{1 y}=p_{10}+p_{11}+p_{12}+p_{13}+\ldots,
\end{aligned}
$$

temos

$$
-\lambda \mathbf{p}+\mu \mathbf{p}_{1 .}=0
$$

Para $s=s^{\prime}=1$ na equação (2.9) temos

$$
\begin{aligned}
& -\left(\lambda+\mu+\mu^{\prime}\right)\left[p_{00}+p_{10}+p_{20}+\ldots+p_{01}+p_{11}+p_{21}+\ldots+p_{02}+p_{12}+p_{22}+\ldots+p_{03}+p_{13}\right. \\
& \left.+p_{23}+\ldots\right]+\mu\left[p_{00}+p_{01}+p_{02}+p_{03}+p_{04}+\ldots\right]+\mu^{\prime}\left[p_{00}+p_{10}+p_{20}+p_{30}+\ldots\right]+\mu^{\prime}\left[p_{01}+\right. \\
& \left.p_{11}+p_{21}+p_{31}+p_{41}+\ldots+p_{02}+p_{12}+p_{22}+p_{32}+\ldots+p_{03}+p_{13}+p_{23}+p_{33}+\ldots\right]+\mu\left[p_{10}+\right. \\
& \left.p_{11}+p_{12}+\ldots+p_{20}+p_{21}+p_{22}+p_{23}+\ldots+p_{30}+p_{31}+p_{32}+\ldots\right]+\lambda\left[\left(p_{00}+p_{01}+p_{02}+\right.\right. \\
& \left.\left.p_{03}+\ldots\right)+\left(p_{11}+p_{12}+p_{13}+p_{14}+\ldots\right)+\left(p_{22}+p_{23}+p_{24}+\ldots\right)+\ldots\right]+\lambda\left[p_{10}+\left(p_{20}+p_{21}\right)+\right. \\
& \left.\left(p_{30}+p_{31}+p_{32}\right)+\ldots\right]=0
\end{aligned}
$$$$
-\left(\lambda+\mu+\mu^{\prime}\right) \sum_{x=0}^{\infty} \sum_{y=0}^{\infty} p_{x y}+\mu^{\prime} \sum_{x=0}^{\infty} \sum_{y=0}^{\infty} p_{x y}+\mu \sum_{x=0}^{\infty} \sum_{y=0}^{\infty} p_{x y}+\lambda \sum_{x=0}^{\infty} \sum_{y=0}^{\infty} p_{x y}=0
$$

Como

$$
\sum_{x=0}^{\infty} \sum_{y=0}^{\infty} p_{x y}=1
$$

temos

$$
0=0
$$


Calculando-se na equação (2.9) da função geratriz de probabilidade do sistema a derivada em relação a s, temos

$$
\begin{aligned}
& -\left(\lambda+\mu+\mu^{\prime}\right)\left[p_{10}+2 p_{20} s+3 p_{30} s^{2}+4 p_{40} s^{3}+\ldots+p_{11} s^{\prime}+2 p_{21} s s^{\prime}+3 p_{31} s^{2} s^{\prime}+\ldots+p_{12} s^{\prime}+\right. \\
& \left.2 p_{22} s s^{\prime 2}+\ldots\right]+\mu^{\prime}\left[p_{10}+2 p_{20} s+3 p_{30} s^{2}+\ldots\right]+\mu^{\prime}\left[p_{11}+2 p_{21} s+3 p_{31} s^{2}+\ldots+p_{12} s^{\prime}+\right. \\
& \left.2 p_{22} s s^{\prime}+3 p_{32} s^{2} s^{\prime}+p_{13} s^{\prime 2}+2 p_{23} s s^{\prime 2}+3 p_{33} s^{2} s^{\prime 2}+\ldots\right]+\mu\left[p_{20}+p_{21} s^{\prime}+p_{22} s^{2}+p_{23} s^{\prime 3}+\right. \\
& \left.\ldots+2 p_{30} s+2 p_{31} s s^{\prime}+2 p_{32} s s^{\prime 2}+\ldots+3 p_{40} s^{2}+3 p_{41} s^{2} s^{\prime}+3 p_{42} s^{2} s^{\prime 2}+\ldots\right]+\lambda\left[p_{00}+p_{01} s^{\prime}+\right. \\
& p_{02} s^{\prime 2}+p_{03} s^{\prime 3}+\ldots+2 s\left(p_{11} s^{\prime}+p_{12} s^{\prime 2}+p_{13} s^{\prime 3}+p_{14} s^{4}+\ldots\right)+3 s^{2}\left(p_{22} s^{\prime 2}+p_{23} s^{\prime 3}+\right. \\
& \left.\left.p_{24} s^{\prime 4}+\ldots\right)+\ldots\right]+\lambda\left[s^{\prime} p_{10}+2 s s^{\prime}\left(p_{20}+p_{21} s^{\prime}\right)+3 s^{2} s^{\prime}\left(p_{30}+p_{31} s^{\prime}+p_{32} s^{\prime 2}\right)+\ldots\right]=0
\end{aligned}
$$

Substituindo-se $s=s^{\prime}=0$ na equação (2.13) temos

$$
-\left(\lambda+\mu+\mu^{\prime}\right) p_{10}+\mu^{\prime} p_{10}+\mu^{\prime} p_{11}+\mu p_{20}+\lambda p_{00}=0
$$

logo,

$$
-\lambda \mathbf{p}_{10}-\mu \mathbf{p}_{10}+\mu^{\prime} \mathbf{p}_{11}+\mu \mathbf{p}_{20}+\lambda \mathbf{p}_{00}=0
$$

Para $s=1$ e $s^{\prime}=0$ na equação (2.13) temos

$$
\begin{aligned}
& -\left(\lambda+\mu+\mu^{\prime}\right)\left[p_{10}+2 p_{20}+3 p_{30}+4 p_{40}+\ldots\right]+\mu^{\prime}\left(p_{10}+2 p_{20}+3 p_{30}+\ldots\right)+\mu^{\prime}\left(p_{11}+2 p_{21}+\right. \\
& \left.3 p_{31}+\ldots\right)+\mu\left(p_{20}+2 p_{30}+3 p_{40}+\ldots\right)+\lambda p_{00}=0,
\end{aligned}
$$

Fazendo-se

$$
\begin{aligned}
& p^{\prime} m_{0}=\sum_{x=0}^{\infty} x p_{x 0}=p_{10}+2 p_{20}+3 p_{30}+\ldots, \\
& p_{.1} m_{1}=\sum_{x=0}^{\infty} x p_{x 1}=p_{11}+2 p_{21}+3 p_{31}+\ldots,
\end{aligned}
$$

temos

$$
-\lambda \mathbf{p}^{\prime} \mathbf{m}_{0}+\mu^{\prime} \mathbf{p} .1 \mathbf{m}_{1}-\mu \mathbf{p}^{\prime}+\mu \mathbf{p}_{00}+\lambda \mathbf{p}_{00}=0 .
$$


Para $s=0$ e $s^{\prime}=1$ na equação (2.13) temos

$$
\begin{aligned}
& -\left(\lambda+\mu+\mu^{\prime}\right)\left[p_{10}+p_{11}+p_{12}+p_{13}+\ldots\right]+\mu^{\prime} p_{10}+\mu^{\prime}\left(p_{11}+p_{12}+p_{13}+\ldots\right)+\mu\left(p_{20}+p_{21}+\right. \\
& \left.p_{22}+\ldots\right)+\lambda\left(p_{00}+p_{01}+p_{02}+\ldots\right)+\lambda p_{10}=0,
\end{aligned}
$$

como

$$
\begin{aligned}
& p_{1 .}=\sum_{y=0}^{\infty} p_{1 y}=p_{10}+p_{11}+p_{12}+\ldots \\
& p_{2 .}=\sum_{y=0}^{\infty} p_{2 y}=p_{20}+p_{21}+p_{22}+\ldots .
\end{aligned}
$$

Temos

$$
-\lambda \mathbf{p}_{1 .}-\mu \mathbf{p}_{1} .+\mu \mathbf{p}_{2} .+\lambda \mathbf{p}+\lambda \mathbf{p}_{10}=0 .
$$

Para $s=s^{\prime}=1$ na equação (2.13) temos

$$
\begin{aligned}
& -\left(\lambda+\mu+\mu^{\prime}\right)\left(p_{10}+2 p_{20}+3 p_{30}+\ldots+p_{11}+2 p_{21}+3 p_{31}+\ldots\right)+\mu^{\prime}\left(p_{10}+2 p_{20}+3 p_{30}+\ldots\right)+ \\
& \mu^{\prime}\left(p_{11}+2 p_{21}+3 p_{31}+\ldots+p_{12}+2 p_{22}+3 p_{32}+p_{13}+2 p_{23}+3 p_{33}+\ldots\right)+\mu\left[p_{20}+p_{21}+p_{22}+\right. \\
& \left.p_{23}+\ldots+2 p_{30}+2 p_{31}+2 p_{32}+3 p_{40}+3 p_{41}+3 p_{42}+\ldots\right)+\lambda\left[p_{00}+p_{01}+p_{02}+p_{03}+\ldots+\right. \\
& \left.2\left(p_{11}+p_{12}+p_{13}+p_{14}+\ldots\right)+3\left(p_{22}+p_{23}+p_{24}+\ldots\right)+\ldots\right]+\lambda\left[p_{10}+2\left(p_{20}+p_{21}\right)+3\left(p_{30}+\right.\right. \\
& \left.\left.p_{31}+p_{32}\right)+\ldots\right]=0
\end{aligned}
$$

Como

$$
\begin{gathered}
\sum_{x=0}^{\infty} \sum_{y=0}^{\infty} x p_{x y}=p_{10}+2 p_{20}+3 p_{30}+\ldots+p_{11}+2 p_{21}+3 p_{31}+\ldots \\
\sum_{x=0}^{\infty} \sum_{y=0}^{\infty}(x-1) p_{x y}+p=p_{20}+p_{21}+p_{22}+p_{23}+\ldots+2 p_{30}+2 p_{31}+2 p_{32}+3 p_{40} \\
\left.+3 p_{41}+3 p_{42}+\ldots\right)+\ldots
\end{gathered}
$$




$$
\begin{aligned}
& -\left(\lambda+\mu+\mu^{\prime}\right) \sum_{x=0}^{\infty} \sum_{y=0}^{\infty} x p_{x y}+\mu^{\prime} \sum_{x=0}^{\infty} \sum_{y=0}^{\infty} x p_{x y}+\mu\left(\sum_{x=0}^{\infty} \sum_{y=0}^{\infty}(x-1) p_{x y}+p\right)+ \\
& \lambda \sum_{x \leq y}(x+1) p_{x y}+\lambda \sum_{x>y} x p_{x y}=0 \\
& -(\lambda+\mu) \sum_{x=0}^{\infty} \sum_{y=0}^{\infty} x p_{x y}+\mu \sum_{x=0}^{\infty} \sum_{y=0}^{\infty} x p_{x y}-\mu \sum_{x=0}^{\infty} \sum_{y=0}^{\infty} p_{x y}+\mu p \\
& \lambda \sum_{x \leq y} x p_{x y}+\lambda \sum_{x>y} x p_{x y}+\lambda \sum_{x \leq y} p_{x y}=0 \\
& -\lambda \sum_{x=0}^{\infty} \sum_{y=0}^{\infty} x p_{x y}-\mu+\mu p+\lambda \sum_{x=0}^{\infty} \sum_{y=0}^{\infty} x p_{x y}+\lambda \sum_{x \leq y} p_{x y}=0 .
\end{aligned}
$$

Seja

$$
N=\sum_{x \leq y} p_{x y} \quad: \text { a freqüência relativa de juntar-se à fila } 1 .
$$

Logo,

$$
\lambda \mathbf{N}=\boldsymbol{\mu}(\mathbf{1}-\mathbf{p}) .
$$

Calculando-se na equação (2.9) a derivada em relação a $s^{\prime}$, temos

$$
\begin{gathered}
-\left(\lambda+\mu+\mu^{\prime}\right)\left[p_{01}+p_{11} s+p_{21} s^{2}+p_{31} s^{3}+\ldots+2 p_{02} s^{\prime}+2 p_{12} s s^{\prime}+2 p_{22} s^{2} s^{\prime}+\ldots\right]+\mu\left[p_{01}+\right. \\
\left.2 p_{02} s^{\prime}+3 p_{03} s^{\prime 2}+\ldots\right]+\mu^{\prime}\left[p_{02}+p_{12} s+p_{22} s^{2}+p_{32} s^{3}+\ldots+2 p_{03} s^{\prime}+2 p_{13} s s^{\prime}+2 p_{23} s^{2} s^{\prime}+\ldots\right. \\
\left.+3 s^{\prime 2} p_{04}+3 p_{14} s s^{\prime 2}+3 p_{24} s^{2} s^{\prime 2}+\ldots\right]+\mu\left[p_{11}+2 p_{12} s^{\prime}+3 p_{13} s^{\prime 2}+\ldots+p_{21} s+2 p_{22} s s^{\prime}+\right. \\
\left.3 p_{23} s s^{\prime 2}+\ldots\right]+\lambda\left[s\left(p_{01}+2 p_{02} s^{\prime}+3 p_{03} s^{\prime 2}+4 p_{04} s^{\prime 3}+\ldots\right)+s^{2}\left(p_{11}+2 p_{12} s^{\prime}+3 p_{13} s^{\prime 2}+\right.\right. \\
\left.\left.4 p_{14} s^{\prime 3}+\ldots\right)+s^{3}\left(2 p_{22} s^{\prime}+3 p_{23} s^{\prime 2}+4 p_{24} s^{3}+\ldots\right)+\ldots\right]+\lambda\left[s p_{10}+s^{2}\left(p_{20}+2 p_{21} s^{\prime}\right)+\right. \\
\left.s^{3} s^{\prime}\left(p_{30}+2 p_{31} s^{\prime}+3 p_{32} s^{\prime 2}\right)+\ldots\right]=0 .
\end{gathered}
$$

Substituindo-se $s=s^{\prime}=0$ na equação acima temos

$$
-\left(\lambda+\mu+\mu^{\prime}\right) p_{01}+\mu p_{01}+\mu^{\prime} p_{02}+\mu p_{11}=0
$$


temos

$$
-\lambda \mathbf{p}_{01}-\mu^{\prime} \mathbf{p}_{01}+\mu^{\prime} \mathbf{p}_{02}+\mu \mathbf{p}_{11}=0
$$

Substituindo-se $s=1$ e $s^{\prime}=0$ na equação (2.18) temos

$$
\begin{aligned}
& -\left(\lambda+\mu+\mu^{\prime}\right)\left(p_{01}+p_{11}+p_{21}+p_{31}+\ldots\right)+\mu p_{01}+\mu^{\prime}\left(p_{02}+p_{12}+p_{22}+p_{32}+\ldots\right)+\mu\left(p_{11}+\right. \\
& \left.p_{21}+p_{31}+\ldots\right)+\lambda\left(p_{01}+p_{11}\right)+\lambda\left(p_{10}+p_{20}+p_{30}+p_{40}+\ldots\right)=0 \\
& -\left(\lambda+\mu+\mu^{\prime}\right) p_{.1}+\mu p_{01}+\mu^{\prime} p_{.2}+\mu\left(p_{.1}-p_{01}\right)+\lambda\left(p_{01}+p_{11}\right)+\lambda\left(p^{\prime}-p_{00}\right)=0 .
\end{aligned}
$$

Logo

$$
-\lambda \mathbf{p}_{.1}-\mu^{\prime} \mathbf{p}_{.1}+\mu^{\prime} \mathbf{p}_{.2}+\lambda \mathbf{p}^{\prime}-\lambda \mathbf{p}_{00}+\lambda \mathbf{p}_{01}+\lambda \mathbf{p}_{11}=0
$$

Substituindo-se $s=0$ e $s^{\prime}=1$ na equação (2.18) temos

$$
\begin{aligned}
& -\left(\lambda+\mu+\mu^{\prime}\right)\left(p_{01}+2 p_{02}+3 p_{03}+\ldots\right)+\mu\left(p_{01}+2 p_{02}+3 p_{03}+\ldots\right)+\mu^{\prime}\left(p_{02}+2 p_{03}+3 p_{04}+\ldots\right)+ \\
& \mu\left(p_{11}+2 p_{12}+3 p_{13}+\ldots\right)=0, \\
& -\left(\lambda+\mu+\mu^{\prime}\right) p m_{0}^{\prime}+\mu p m_{0}^{\prime}+\mu^{\prime}\left(p m_{0}^{\prime}-p+p_{00}\right)+\mu p_{1 .} m_{1}^{\prime}=0 .
\end{aligned}
$$

Logo

$$
-\lambda \mathbf{p} \mathbf{m}_{0}^{\prime}-\mu^{\prime} \mathbf{p}+\mu^{\prime} \mathbf{p}_{00}+\mu \mathbf{p}_{1} \mathbf{m}_{1}^{\prime}=0
$$

Substituindo-se $s=s^{\prime}=1$ na equação (2.18) temos

$$
\begin{aligned}
& -\left(\lambda+\mu+\mu^{\prime}\right) \sum_{x=0}^{\infty} \sum_{y=0}^{\infty} y p_{x y}+\mu \sum_{y=0}^{\infty} y p_{0 y}+\mu^{\prime}\left(\sum_{x=0}^{\infty} \sum_{y=0}^{\infty}(y-1) p_{x y}+p^{\prime}\right)+\mu\left(\sum_{x=0}^{\infty} \sum_{y=0}^{\infty} y p_{x y}-\right. \\
& \left.\sum_{y=0}^{\infty} y p_{0 y}\right)+\lambda \sum_{x \leq y}^{\infty} y p_{x y}+\lambda \sum_{x>y}^{\infty}(y+1) p_{x y}=0, \\
& -\left(\lambda+\mu+\mu^{\prime}\right) \sum_{x=0}^{\infty} \sum_{y=0}^{\infty} y p_{x y}+\mu^{\prime} \sum_{x=0}^{\infty} \sum_{y=0}^{\infty} y p_{x y}-\mu^{\prime}+\mu^{\prime} p^{\prime}+\mu \sum_{x=0}^{\infty} \sum_{y=0}^{\infty} y p_{x y}+\lambda\left(\sum_{x \leq y}^{\infty} y p_{x y}+\right. \\
& \left.\lambda \sum_{x>y}^{\infty} y p_{x y}\right)+\lambda \sum_{x>y}^{\infty} p_{x y}=0 .
\end{aligned}
$$


Então

$$
-\mu^{\prime}\left(1-p^{\prime}\right)+\lambda \sum_{x>y}^{\infty} p_{x y}=0
$$

Sendo

$$
F=\sum_{x>y}^{\infty} p_{x y} \quad: \text { a freqüência relativa de juntar-se à fila } 2 \text {. }
$$

Logo,

$$
\lambda \mathbf{F}=\boldsymbol{\mu}^{\prime}\left(\mathbf{1}-\mathbf{p}^{\prime}\right)
$$

Calculando-se a segunda derivada em relação a s na equação (2.13) temos

$$
\begin{gathered}
-\left(\lambda+\mu+\mu^{\prime}\right)\left[2 p_{20}+6 p_{30} s+12 p_{40} s^{2}+\ldots+2 p_{21} s^{\prime}+6 p_{31} s s^{\prime}+12 p_{41} s^{2} s^{\prime}+\ldots\right]+\mu^{\prime}\left[2 p_{20}+\right. \\
\left.6 p_{30} s+12 p_{40} s^{2}+\ldots\right]+\mu^{\prime}\left[2 p_{21}+6 p_{31} s+12 p_{41} s^{2}+\ldots+2 p_{22} s^{\prime}+6 p_{32} s s^{\prime}+12 p_{42} s^{2} s^{\prime}+\ldots+\right. \\
2 p_{23} s^{\prime 2}+6 p_{33} s s^{\prime 2}+12 p_{43} s^{2} s^{\prime 2}+\ldots .+\mu\left[2 p_{30}+2 p_{31} s^{\prime}+2 p_{32} s^{\prime 2}+\ldots+6 p_{40} s+6 p_{41} s s^{\prime 2}+\right. \\
\left.6 p_{42} s s^{\prime 2}+\ldots\right]+\lambda\left[2\left(p_{11} s^{\prime}+p_{12} s^{\prime 2}+p_{13} s^{\prime 3}+\ldots\right)+6 s\left(p_{22} s^{\prime 2}+p_{23} s^{\prime 3}+p_{24} s^{\prime 4}+\ldots\right)+\ldots\right]+ \\
\lambda\left[2 s^{\prime}\left(p_{20}+p_{21} s^{\prime}\right)+6 s s^{\prime}\left(p_{30}+p_{31} s^{\prime}+p_{32} s^{\prime 2}\right)+. .\right]=0
\end{gathered}
$$

Substituindo-se $s=s^{\prime}=0$ na equação acima temos

$$
\left.-\left(\lambda+\mu+\mu^{\prime}\right) 2 p_{20}+\mu^{\prime}\right) 2 p_{20}+\mu^{\prime} 2 p_{21}+\mu 2 p_{30}=0
$$

Logo,

$$
-\lambda \mathbf{p}_{20}-\mu \mathbf{p}_{20}+\mu^{\prime} \mathbf{p}_{21}+\mu \mathbf{p}_{20}=0
$$

Substituindo-se $s=1$ e $s^{\prime}=0$ na equação (2.23) temos

$$
\begin{aligned}
& -\left(\lambda+\mu+\mu^{\prime}\right)\left(2 p_{20}+6 p_{30}+12 p_{40}+\ldots\right)+\mu^{\prime}\left(2 p_{20}+6 p_{30}+12 p_{40}+\ldots\right)+ \\
& \mu^{\prime}\left(2 p_{21}+6 p_{31}+12 p_{41}+\ldots\right)+\mu\left[2 p_{30}+6 p_{40}+12 p_{50}+\ldots\right)=0
\end{aligned}
$$


Sendo

$$
\begin{gathered}
p^{\prime} w_{0}=\sum_{x=0}^{\infty}\left(x^{2}-x\right) p_{x 0}=2 p_{20}+6 p_{30}+12 p_{40}+\ldots \\
p_{.1} w_{1}=\sum_{x=0}^{\infty}\left(x^{2}-x\right) p_{x 1}=2 p_{21}+6 p_{30}+12 p_{40}+\ldots \\
p^{\prime} w_{0}-2 p^{\prime} m_{0}+2 p^{\prime}-2 p_{00}=2 p_{30}+6 p_{40}+12 p_{50}+\ldots \\
-\left(\lambda+\mu+\mu^{\prime}\right) p^{\prime} w_{0}+\mu^{\prime} p^{\prime} w_{0}+\mu^{\prime} p_{.1} w_{1}+\mu^{\prime}\left(p^{\prime} w_{0}-2 p^{\prime} m_{0}+2 p^{\prime}-2 p_{00}\right)=0
\end{gathered}
$$

logo,

$$
-\lambda \mathbf{p}^{\prime} \mathrm{w}_{0}+\mu^{\prime} \mathrm{p}_{.1} \mathrm{w}_{1}-2 \mu \mathrm{p}^{\prime} \mathrm{m}_{0}+2 \mu \mathrm{p}^{\prime}-2 \mu \mathrm{p}_{00}=0 .
$$

Substituindo-se $s=0$ e $s^{\prime}=1$ na equação (2.23) temos

$$
\begin{gathered}
-\left(\lambda+\mu+\mu^{\prime}\right)\left(2 p_{20}+2 p_{21}+2 p_{22}+\ldots\right)+\mu^{\prime} 2 p_{20}+\mu^{\prime}\left(2 p_{21}+2 p_{22}+\ldots\right)+\mu\left(2 p_{30}+2 p_{31}+\right. \\
\left.2 p_{32}+\ldots\right)+\lambda 2\left(p_{11}+p_{12}+\ldots\right)+\lambda 2\left(p_{20}+p_{21}\right)=0 \\
-\left(\lambda+\mu+\mu^{\prime}\right) p_{2 .}+\mu^{\prime} p_{2 .}+\mu p_{3 .}+\lambda\left(p_{1 .}-p_{10}\right)+\lambda p_{20}+\lambda p_{21}=0 .
\end{gathered}
$$

Logo,

$$
-\lambda \mathbf{p}_{2}-\mu \mathbf{p}_{2 .}+\mu \mathbf{p}_{3 .}+\lambda \mathbf{p}_{1 .}-\lambda \mathbf{p}_{10}+\lambda \mathbf{p}_{20}+\lambda \mathbf{p}_{21}=0
$$

Substituindo-se $s=s^{\prime}=1$ na equação (2.23) temos

$$
\begin{aligned}
& -\left(\lambda+\mu+\mu^{\prime}\right) \sum_{x=0}^{\infty} \sum_{y=0}^{\infty} x(x-1) p_{x y}+\mu^{\prime} \sum_{x=0}^{\infty} x(x-1) p_{x 0}+\mu^{\prime} \sum_{x=0}^{\infty} \sum_{y=0}^{\infty} x(x-1) p_{x y}- \\
& \mu^{\prime} \sum_{x=0}^{\infty} x(x-1) p_{x=0}+\mu \sum_{x=0}^{\infty} \sum_{y=0}^{\infty}(x-1)(x-2) p_{x y}-2 \mu \sum_{y=0}^{\infty} p_{0 y}+D_{x y}(1,1)=0, \\
& -\lambda \sum_{x=0}^{\infty} \sum_{y=0}^{\infty} x^{2} p_{x y}+\lambda \sum_{x=0}^{\infty} \sum_{y=0}^{\infty} x p_{x y}-\mu \sum_{x=0}^{\infty} \sum_{y=0}^{\infty} x^{2} p_{x y}+\mu \sum_{x=0}^{\infty} \sum_{y=0}^{\infty} x p_{x y}+\mu \sum_{x=0}^{\infty} \sum_{y=0}^{\infty} x^{2} p_{x y}- \\
& 3 \mu \sum_{x=0}^{\infty} \sum_{y=0}^{\infty} x p_{x y}+2 \mu \sum_{x=0}^{\infty} \sum_{y=0}^{\infty} p_{x y}-2 \mu \sum_{y=0}^{\infty} p_{0 y y}+D_{x x}(1,1)=0 .
\end{aligned}
$$


Sendo

$$
\begin{gathered}
\sum_{s=0}^{\infty} \sum_{y=0}^{\infty} x^{2} p_{x y}=\nu+m^{2}, \\
\sum_{x=0}^{\infty} \sum_{y=0}^{\infty} x p_{x y}=m, \\
-\lambda\left(\nu+m^{2}-m\right)-2 \mu m+2 \mu p-2 \mu+D_{s o}(1,1)=0,
\end{gathered}
$$

logo,

$$
D_{w}(1,1)=\lambda w+2 \mu m-2 \mu+2 \mu p \text {. }
$$

Calculando-se a segunda derivada em relação a $s^{\prime}$ na equação (2.18) temos

$$
\begin{aligned}
& -\left(\lambda+\mu+\mu^{\prime}\right)\left[2 p_{02}+2 p_{12} s+2 p_{22} s^{2}+\ldots+6 p_{03} s^{\prime}+6 p_{13} s s^{\prime}+6 p_{23} s^{2} s^{\prime}+\ldots\right]+\mu\left[2 p_{02}+\right. \\
& \left.6 p_{03} s^{\prime}+12 p_{04} s^{\prime 2}+\ldots\right]+\mu^{\prime}\left[2 p_{03}+2 p_{13} s+2 p_{23} s^{2}+\ldots+6 p_{04} s^{\prime}+6 p_{14} s s^{\prime}+6 p_{24} s^{2} s^{\prime}+\ldots\right]+ \\
& \mu\left[2 p_{12}+6 p_{13} s^{\prime}+12 p_{14} s^{\prime 2}+\ldots+2 p_{22} s+6 p_{23} s s^{\prime}+12 p_{24} s s^{\prime 2}+\ldots\right]+\lambda\left[s \left(2 p_{02}+6 p_{03} s^{\prime}+\right.\right. \\
& \left.12 p_{04} s^{\prime 2}+\ldots\right)+s^{2}\left(2 p_{12}+6 p_{13} s^{\prime}+12 p_{14} s^{\prime 2}+\ldots\right)+s^{3}\left(2 p_{22}+6 p_{23} s^{\prime}+12 p_{24} s^{3}+\ldots\right)+ \\
& \left.+s^{4}\left(6 p_{33} s^{\prime}+12 p_{34} s^{\prime 2}+20 p_{44} s^{\prime 3}+\ldots\right)+\ldots\right]+\lambda\left[s^{2} 2 p_{21}+s^{8}\left(2 p_{31}+6 p_{32} s^{\prime}\right)+\ldots\right]=0
\end{aligned}
$$

Substituindo-se $s=s^{\prime}=0$ na equação acima temos

$$
-\left(\lambda+\mu+\mu^{\prime}\right) 2 p_{02}+\mu 2 p_{02}+\mu^{\prime} 2 p_{03}+\mu p_{12}=0,
$$

Logo,

$$
-\lambda \mathbf{p}_{02}-\mu^{\prime} \mathbf{p}_{02}+\mu^{\prime} \mathbf{p}_{03}+\mu \mathbf{p}_{12}=0
$$

Substituindo-se $s=1$ e $s^{\prime}=0$ na equação (2.28) temos

$$
\begin{aligned}
& -\left(\lambda+\mu+\mu^{\prime}\right) 2\left(p_{02}+p_{12}+p_{22}+\ldots\right)+2 \mu p_{02}+2 \mu^{\prime}\left(p_{03}+p_{13}+p_{23}+\ldots\right)+2 \mu\left(p_{12}+p_{22}+\right. \\
& \left.p_{23}+\ldots\right)+2 \lambda\left(p_{02}+p_{12}+p_{22}\right)+2 \lambda\left(p_{21}+p_{31}+p_{41}+\ldots\right)=0,
\end{aligned}
$$


2.3 Análise do Modelo

25

$$
-\left(\lambda+\mu+\mu^{\prime}\right) p_{.2}+\mu p_{.2}+\mu^{\prime} p_{.3}+\lambda\left(p_{02}+p_{12}+p_{22}\right)+\lambda\left(p_{.1}-p_{01}-p_{11}\right)=0
$$

logo,

$$
-\lambda \mathbf{p}_{.2}-\mu^{\prime} \mathbf{p} .2+\mu^{\prime} \mathbf{p} .3+\lambda \mathbf{p}_{.1}-\lambda \mathbf{p}_{01}-\lambda \mathbf{p}_{11}+\lambda \mathbf{p}_{02}+\lambda \mathbf{p}_{12}+\lambda \mathbf{p}_{22}=\mathbf{0}
$$

Substituindo-se $s=0$ e $s^{\prime}=1$ na equação (2.28), temos

$$
\begin{aligned}
& -\left(\lambda+\mu+\mu^{\prime}\right)\left(2 p_{02}+6 p_{03}+12 p_{04}+\ldots\right)+\mu\left(2 p_{02}+6 p_{03}+12 p_{04}+\ldots\right)+\mu^{\prime}\left(2 p_{03}+6 p_{04}+\right. \\
& \left.12 p_{05}+\ldots\right)+\mu\left(2 p_{12}+6 p_{13}+12 p_{14}+\ldots\right)=0 .
\end{aligned}
$$

Sendo

$$
\begin{gathered}
p w_{0}^{\prime}=\sum_{y=0}^{\infty}\left(y^{2}-y\right) p_{0 y}=2 p_{02}+6 p_{03}+12 p_{04}+\ldots \\
p_{1 .} w_{1}^{\prime}=\sum_{y=0}^{\infty}\left(y^{2}-y\right) p_{0 y}=2 p_{12}+6 p_{13}+12 p_{14}+\ldots, \\
p w_{0}^{\prime}-2 p m+2 p-2 p_{00}=2 p_{03}+6 p_{04}+12 p_{05}+\ldots, \\
-\left(\lambda+\mu+\mu^{\prime}\right) p w_{0}^{\prime}+\mu p w_{0}^{\prime}+\mu^{\prime}\left(p w w_{0}^{\prime}-2 p m^{\prime}+2 p-2 p_{00}\right)+\mu p_{1 .} w_{1}^{\prime}=0
\end{gathered}
$$

logo,

$$
-\lambda p w_{0}^{\prime}-2 \mu^{\prime} \mathrm{pm}_{0}^{\prime}+2 \mu^{\prime} \mathrm{p}-2 \mu^{\prime} \mathrm{p}_{00}+\mu \mathrm{p}_{1} . \mathrm{w}_{1}^{\prime}=0
$$

Substituindo-se $s=s^{\prime}=1$ na equação (2.28), temos

$$
\begin{aligned}
& -\left(\lambda+\mu+\mu^{\prime}\right)\left(2 p_{02}+2 p_{12}+2 p_{22}+\ldots+6 p_{03}+6 p_{13}+6 p_{23}+\ldots\right)+\mu\left(2 p_{02}+6 p_{03}+12 p_{04}\right. \\
& +\ldots)+\mu^{\prime}\left(2 p_{03}+2 p_{13}+2 p_{23}+\ldots+6 p_{04}+6 p_{14}+6 p_{24}+\ldots\right)+\mu\left(2 p_{12}+6 p_{13}+12 p_{14}+\ldots\right. \\
& \left.+2 p_{22}+6 p_{23}+12 p_{24}+\ldots\right)+\lambda\left(2 p_{02}+6 p_{03}+12 p_{04}+\ldots+2 p_{12}+6 p_{13}+12 p_{14}+\ldots+2 p_{22}\right. \\
& \left.+6 p_{23}+12 p_{24}+\ldots\right)+\lambda\left(2 p_{21}+2 p_{31}+6 p_{32}+2 p_{41}+6 p_{42}+12 p_{43}+\ldots\right)=0
\end{aligned}
$$




$$
\begin{aligned}
& -\left(\lambda+\mu+\mu^{\prime}\right) \sum_{x=0}^{\infty} \sum_{y=0}^{\infty} y(y-1) p_{x y}+\mu \sum_{y=0}^{\infty} y(y-1) p_{0 y}+\mu^{\prime}\left(\sum_{x=0}^{\infty} \sum_{y=0}^{\infty}(y-1)(y-2) p_{x y}-\right. \\
& \left.2 \sum_{x=0}^{\infty} p_{x=0}\right)+\mu\left(\sum_{x=0}^{\infty} \sum_{y=0}^{\infty} y(y-1)-\sum_{y=0}^{\infty} y(y-1)\right)+\lambda \sum_{x \leq y}^{\infty} y(y-1) p_{x y}+\lambda \sum_{x>y}^{\infty} y(y+1) p_{x y}=0, \\
& -\lambda\left(\sum_{x=0}^{\infty} \sum_{y=0}^{\infty} y^{2} p_{x y}-\sum_{x=0}^{\infty} \sum_{y=0}^{\infty} y p_{x y}\right)-2 \sum_{x=0}^{\infty} \sum_{y=0}^{\infty} y p_{x y}+2 \mu^{\prime} \sum_{x=0}^{\infty} \sum_{y=0}^{\infty} p_{x y}-2 \mu^{\prime} \sum_{x=0}^{\infty} p_{x 0}+D_{x^{\prime} s^{\prime}}(1,1)=0,
\end{aligned}
$$

sendo

$$
\begin{aligned}
& \sum_{x=0}^{\infty} \sum_{y=0}^{\infty} y^{2} p_{x y}=v^{\prime}+m^{2} \\
& \sum_{x=0}^{\infty} \sum_{y=0}^{\infty} y p_{x y}=m^{\prime} \\
& D_{x \prime}(1,1)=\lambda \sum_{x \leq y}^{\infty} y(y-1) p_{x y}+\lambda \sum_{x>y}^{\infty} y(y+1) p_{x y},
\end{aligned}
$$

logo,

$$
D_{s^{\prime}}(1,1)=\lambda w^{\prime}+2 \mu^{\prime} m^{\prime}-2 \mu^{\prime}+2 \mu^{\prime} p^{\prime}
$$

Calculando-se a derivada mista de $s$ em relação a $s^{\prime}$ na equação (2.13) temos

$$
\begin{aligned}
& -\left(\lambda+\mu+\mu^{\prime}\right)\left(p_{11}+2 p_{21} s+3 p_{31} s^{2}+\ldots+2 p_{12} s^{\prime}+4 p_{22} s s^{\prime}+6 p_{32} s^{2} s^{\prime}+\ldots+3 p_{13} s^{\prime 2}+\right. \\
& \left.6 p_{23} s s^{\prime 2}+9 p_{33} s^{2} s^{\prime 2}+\ldots+4 p_{14} s^{3}+8 p_{24} s s^{\prime 3}+12 p_{34} s^{2} s^{\prime 3}+\ldots\right)+\mu^{\prime}\left(p_{12}+2 p_{22} s+\right. \\
& \left.3 p_{32} s^{2}+\ldots+2 p_{13} s^{\prime}+4 p_{23} s s^{\prime}+6 p_{33} s^{s} s^{\prime}+\ldots+3 p_{14} s^{\prime 2}+6 p_{24} s s^{\prime 2}+9 p_{34} s^{2} s^{\prime 2}+\ldots\right)+ \\
& \mu\left(p_{21}+2 p_{22} s^{\prime}+3 p_{23} s^{\prime 2}+\ldots+2 p_{31} s+4 p_{32} s s^{\prime}+6 p_{33} s s^{\prime 2}+\ldots+3 p_{41} s^{2}+6 p_{42} s^{2} s^{\prime}+\right. \\
& \left.9 p_{43} s^{2} s^{\prime 2}+\ldots\right)+\lambda\left(p_{01}+2 p_{02} s^{\prime}+3 p_{03} s^{\prime 2}+\ldots 2 s\left(p_{11}+2 p_{12} s^{\prime}+3 p_{13} s^{\prime 2}+\ldots\right)+3 s^{2}\left(2 p_{22} s^{\prime}+\right.\right. \\
& \left.\left.3 p_{23} s^{\prime 2}+\ldots\right)+\ldots\right)+\lambda\left(p_{10}+2 s\left(p_{20}+2 p_{21} s^{\prime}\right)+3 s^{2}\left(p_{30}+2 p_{31} s^{\prime}+3 p_{32} s^{2}\right)+\ldots\right)=0 .
\end{aligned}
$$

Substituindo-se $s=s^{\prime}=0$ na equação acima temos

$$
-\left(\lambda+\mu+\mu^{\prime}\right) \mathbf{p}_{11}+\mu^{\prime} \mathbf{p}_{12}+\mu \mathbf{p}_{21}+\lambda\left(\mathbf{p}_{01}+\mathbf{p}_{10}\right)=0
$$


Substituindo-se $s=1$ e $s^{\prime}=0$ na equação (2.33) temos

$$
\begin{aligned}
& -\left(\lambda+\mu+\mu^{\prime}\right)\left(p_{11}+2 p_{21}+3 p_{31}+\ldots\right)+\mu^{\prime}\left(p_{12}+2 p_{22}+3 p_{32}+\ldots\right)+\mu\left(p_{21}+2 p_{31}+\right. \\
& \left.3 p_{41}+\ldots\right)+\lambda\left(p_{01}+2 p_{11}\right)+\lambda\left(p_{10}+2 p_{30}+3 p_{30}+\ldots\right)=0, \\
& -\left(\lambda+\mu+\mu^{\prime}\right) p_{.1} m_{1}+\mu^{\prime} p_{.2} m_{2}+\mu\left(p_{.1} m_{1}-p_{.1}+p_{01}\right)+\lambda\left(p_{01}+2 p_{11}+p^{\prime} m_{0}\right)=0,
\end{aligned}
$$

logo,

$$
-\left(\lambda+\mu^{\prime}\right) \mathbf{p}_{.1} \mathbf{m}_{1}+\mu^{\prime} \mathbf{p}_{.2} \mathbf{m}_{2}-\mu \mathbf{p}_{.1}+\mu \mathbf{p}_{01}+\lambda\left(\mathbf{p}_{01}+2 \mathbf{p}_{11}+\mathbf{p}^{\prime} \mathbf{m}_{0}\right)=0
$$

Substituindo-se $s=0$ e $s^{\prime}=1$ na equação (2.33) temos

$$
\begin{aligned}
& -\left(\lambda+\mu+\mu^{\prime}\right)\left(p_{11}+2 p_{12}+3 p_{13}+\ldots\right)+\mu^{\prime}\left(p_{12}+2 p_{13}+3 p_{14}+\ldots\right)+\mu\left(p_{21}+2 p_{22}+\right. \\
& \left.3 p_{23}+\ldots\right)+\lambda\left(p_{01}+2 p_{02}+3 p_{03}+\ldots\right)+\lambda p_{10}=0 \\
& -\left(\lambda+\mu+\mu^{\prime}\right) p_{1 .} m_{1}^{\prime}-\mu^{\prime}\left(p_{1} . m_{1}^{\prime}-p_{1 .}+p_{10}\right)+\mu p_{2} . m_{2}^{\prime}+\lambda\left(p_{10}+p m_{0}^{\prime}\right)=0,
\end{aligned}
$$

logo,

$$
-(\lambda+\mu) \mathbf{p}_{1} \cdot \mathbf{m}_{1}^{\prime}+\mu \mathbf{p}_{2} \cdot \mathbf{m}_{2}^{\prime}-\mu^{\prime} \mathbf{p}_{1 .}+\mu^{\prime} \mathbf{p}_{10}+\lambda\left(\mathbf{p}_{10}+\mathbf{p m}_{0}^{\prime}\right)=0
$$

Substituindo-se $s=s^{\prime}=1$ na equação (2.33), temos

$$
\begin{aligned}
& -\left(\lambda+\mu+\mu^{\prime}\right) \sum_{x=0}^{\infty} \sum_{y=0}^{\infty} x y p_{x y}+\mu^{\prime}\left(\sum_{x=0}^{\infty} \sum_{y=0}^{\infty} x(y-1)+\sum_{x=0}^{\infty} x p_{x 0}\right)+\mu\left(\sum_{x=0}^{\infty} \sum_{y=0}^{\infty}(x-1) y p_{x y}+\right. \\
& \left.\sum_{y=0}^{\infty} y p_{0 y}\right)+\lambda \sum_{x \leq y}^{\infty}(x+1) p_{x y}+\lambda \sum_{x>y}^{\infty} x(y+1) p_{x y}=0 \\
& -\left(\lambda+\mu+\mu^{\prime}\right) \sum_{x=0}^{\infty} \sum_{y=0}^{\infty} x y p_{x y}+\mu^{\prime} \sum_{x=0}^{\infty} \sum_{y=0}^{\infty} x y p_{x y}-\mu^{\prime} \sum_{x=0}^{\infty} \sum_{y=0}^{\infty} x y p_{x y}+\mu^{\prime} \sum_{x=0}^{\infty} x p_{x 0}+ \\
& \mu \sum_{x=0}^{\infty} \sum_{y=0}^{\infty} x y p_{x y}-\mu \sum_{x=0}^{\infty} \sum_{y=0}^{\infty} y p_{x y}+\mu \sum_{y=0}^{\infty} y p_{0 y}+D_{x x^{\prime}}(1,1)=0, \\
& -\lambda \sum_{x=0}^{\infty} \sum_{y=0}^{\infty} x y p_{x y}-\mu^{\prime} \sum_{x=0}^{\infty} \sum_{y=0}^{\infty} x p_{x y}+\mu^{\prime} \sum_{x=0}^{\infty} x p_{x 0}-\mu \sum_{x=0}^{\infty} \sum_{y=0}^{\infty} y p_{x y}+\mu \sum_{y=0}^{\infty} y p_{0 y}+D_{x y^{\prime}}(1,1)=0 .
\end{aligned}
$$


Sendo

$$
\begin{aligned}
& m=\sum_{x=0}^{\infty} x p_{x .}=\sum_{x=0}^{\infty} \sum_{y=0}^{\infty} x p_{x y}, \quad m_{0}=E\left[X \mid X^{\prime}=0\right]=\sum_{x=0}^{\infty} x p_{x 0} \\
& m^{\prime}=\sum_{y=0}^{\infty} y p_{. y}=\sum_{x=0}^{\infty} \sum_{y=0}^{\infty} y p_{x y}, \quad m_{0}^{\prime}=E\left[X^{\prime} \mid X=0\right]=\sum_{y=0}^{\infty} y p_{0 y}, \\
& r=\frac{C o v\left(X, X^{\prime}\right)}{\sigma \sigma^{\prime}} \rightarrow \operatorname{Cov}\left(X, X^{\prime}\right)=r \sigma \sigma^{\prime},
\end{aligned}
$$

r: coeficiente de correlação do processo $X(t)$ e $X^{\prime}(t)$;

$\sigma$ : desvio padrão do número de clientes na fila 1 em equilíbrio;

$\sigma^{\prime}$ : desvio padrão do número de clientes na fila 2 em equilíbrio.

Lembrando-se que

$$
\operatorname{Cov}\left(X, X^{\prime}\right)=E\left(X X^{\prime}\right)-E(X) E\left(X^{\prime}\right)=\sum_{x=0}^{\infty} \sum_{y=0}^{\infty} x y p_{x y}-\sum_{x=0}^{\infty} \sum_{y=0}^{\infty} x p_{x y} \sum_{x=0}^{\infty} \sum_{y=0}^{\infty} y p_{x y},
$$

então

$$
\begin{gathered}
\sum_{x=0}^{\infty} \sum_{y=0}^{\infty} x y p_{x y}=r \sigma \sigma^{\prime}+m m^{\prime} \\
-\lambda\left(r \sigma \sigma^{\prime}+m m^{\prime}\right)-\mu^{\prime} m+\mu^{\prime} m_{0}-\mu m^{\prime}+\mu m_{0}+D_{s s^{\prime}}(1,1)=0 .
\end{gathered}
$$

Logo,

$$
\mathbf{D}_{\mathbf{s}}(\mathbf{1}, \mathbf{1})=\lambda \mathrm{r} \sigma \sigma^{\prime}+\lambda \mathbf{m m}^{\prime}+\mu \mathrm{m}+\mu \mathbf{m}^{\prime}-\mu^{\prime} \mathbf{m}_{0}-\mu \mathbf{m}_{0}^{\prime}
$$




\subsection{Distribuição do número de Clientes nas Filas 1 e 2 e no Sistema}

Utilizaremos a seguinte notação para distribuição condicional de probabilidade:

$$
\begin{array}{ll}
p_{. y} P_{x y}=\sum_{i=0}^{x} p_{i y}, & Q_{x y}=1-P_{x y}, \\
p_{x .} P_{x y}^{\prime}=\sum_{j=0}^{y} p_{x j}, & Q_{x y}^{\prime}=1-P_{x y}^{\prime},
\end{array}
$$

onde

$$
P_{x y}=P\left(X \leq x \mid X^{\prime}=y\right) \quad \text { e } \quad P_{x y}^{\prime}=P\left(X^{\prime} \leq y \mid X=x\right)
$$

Da equação (2.12), dividindo-se por $\mu$ e isolando-se o termo $p_{1 .,}$ obtemos

$$
\mathbf{p}_{1 .}=\rho \mathbf{p}
$$

onde

$$
p=p_{00}+p_{01}+p_{02}+\ldots=\sum_{j=0}^{\infty} p_{0 j} .
$$

Da equação (2.16), dividindo-se por $\mu$ e isolando-se $p_{2 .}$, obtemos

$$
\begin{aligned}
& -\rho p_{1 .}-p_{1 .}+p_{2 .}+p_{1 .}+\rho p_{10}=0 \\
& p_{2 .}=\rho\left(p_{1 .}-p_{10}\right) .
\end{aligned}
$$

Por definição temos que $\sum_{j=0}^{y} p_{1 y}=p_{1} . P_{1 y}^{\prime}$.

Para $y=0$ temos $p_{10}=p_{1} P_{10}^{\prime}$

$$
p_{2 .}=\rho\left(p_{1 .}-p_{1 .} P_{10}^{\prime}\right)=\rho p_{1 .}\left(1-P_{10}^{\prime}\right),
$$

então

$$
\mathbf{p}_{2 .}=\rho \mathbf{p}_{1} . \mathbf{Q}_{10}^{\prime} \text {. }
$$

Da equação (2.26), dividindo-se por $\mu$ e isolando-se o termo $p_{3 .}$, temos

$$
\begin{aligned}
& -\rho p_{2 .}-p_{2 .}+p_{3 .}+p_{2 .}+\rho p_{20}+\rho p_{21}=0, \\
& p_{3 .}=\rho\left[p_{2 .}-\left(p_{20}+p_{21}\right)\right] .
\end{aligned}
$$


Para $x=2$ temos da notação $\sum_{j=0}^{y} p_{2 j}=p_{2 .} P_{2 y}^{\prime}$.

Tomando-se $y=1$ temos $p_{20}+p_{21}=p_{2 .} P_{21}^{\prime}$

$$
p_{3 .}=\rho\left(p_{2 .}-p_{2 .} P_{21}^{\prime}\right)=\rho p_{2 .}\left(1-P_{21}^{\prime}\right),
$$

então

$$
\mathbf{p}_{\mathbf{s}}=\rho \mathbf{p}_{2} . \mathbf{Q}_{21}^{\prime}
$$

Generalizando-se, obtemos

$$
p_{x .}=\rho p_{x \rightarrow 1} . Q_{x-1, x-2}^{\prime}
$$

Para a fila 2, fazendo-se o mesmo procedimento feito na fila $1 \mathrm{com}$ as equações (2.11), (2.20) e (2.30), obtemos

$$
\mathbf{p}_{. \mathbf{y}}=\rho^{\prime} \mathbf{Q}_{\mathbf{y}-1, \mathbf{y - 1}}^{\prime} \mathbf{p}_{., \mathbf{y}-1}
$$

Uma relação importante é obtida na equação (2.2) para todo $x$ e $y$. Temos a seguinte expressão para um valor $n$ fixado:

$$
\lambda \mathrm{p}_{\mathrm{nn}}=\mu \mathbf{p}_{\mathrm{n}+1, .} \mathbf{P}_{\mathrm{n}+1, \mathrm{n}}^{\prime}+\mu^{\prime} \mathbf{p}_{. \mathrm{n}+1} \mathbf{P}_{\mathrm{n}, \mathrm{n}+1} \quad(\mathrm{n}=0,1,2, \ldots)
$$

Somando-se para todo $n$, obtemos

$$
\lambda \sum_{x=y} p_{x y}=\mu \sum_{x>y} p_{x y}+\mu^{\prime} \sum_{x<y} p_{x y} \quad(n=0,1,2, \ldots)
$$

Por outro lado, tomando-se $x+y=n$, obtemos

$$
\lambda \sum_{\mathbf{x}+\mathbf{y}=\mathbf{n}} \mathbf{p}_{\mathbf{x y}}=\left(\mu+\mu^{\prime}\right)\left(\mathbf{p}_{\mathrm{n}, \mathrm{n}-1}\right)+\mu \mathbf{p}_{\mathrm{n}+1,0}+\mu^{\prime} \mathbf{p}_{0, \mathrm{n}+1} \quad(n=0,1, \ldots)
$$

Desta forma concluímos que a probabilidade de haver $x$ clientes no sistema (denotamos por $\pi_{x}$ ) é dada pela seguinte expressão:

$$
\pi_{\mathbf{x}}=\frac{1}{\rho}\left(\pi_{\mathbf{x}+1}-\mathbf{p}_{0, \mathbf{x}+1}\right)+\frac{1}{\rho^{\prime}}\left(\pi_{\mathbf{x}+1}-\mathbf{p}_{\mathbf{x}+1,0}\right)
$$




\subsection{Resultados Numéricos}

O sistema de filas $M /(M / 1)^{2}$, apresentado neste capítulo, permite obter soluções para a distribuição de equilíbrio para um número qualquer de clientes no sistema. Entretanto, para ilustração numérica apresentamos a distribuição de equilíbrio do sistema com um número limitado de clientes. Primeiramente, efetuamos uma simulação do sistema em equilíbrio com trinta rodadas e 1000 clientes atendidos em cada rodada, com o objetivo de dimensionar o número máximo de clientes no sistema. Nessa simulação utilizamos os parâmetros: taxa de chegada $\lambda=5$, para taxa de serviço de cada um dos servidores $\mu_{1}=\mu_{2}=6$, e após essas rodadas feitas conclú́mos que o valor igual a dez pode reproduzir adequadamente o sistema de filas. Utilizamos o pacote de simulação GPSS/H (General Purpose Simulation System, version H) para simular o sistema de fila $M /(M / 1)^{2}$.

Deste modo, a distribuição de equilíbrio para o número total de clientes no sistema, conforme a equação (1.46), com dez clientes, no sistema obtivemos com o algoritmo apresentado no Apêndice A que, executado no software Mathematica, apresenta os seguintes resultados.

Para $\lambda=5, \mu_{1}=\mu_{2}=6$ temos

$\pi=(0,4349 ; 0,3427 ; 0,1419 ; 0,0518 ; 0,0185 ; 0,0066 ; 0,0023 ; 0,0009 ; 0,0003 ; 0.0001 ; 0,0000)$

Para $\lambda=10,8 \mu_{1}=\mu_{2}=6$ temos

$$
\pi=(0,0914 ; 0,1206 ; 0,1231 ; 0,1152 ; 0,1050 ; 0,0948 ; 0,0854 ; 0,0769 ; 0,0692 ; 0,0623 ; 0,0561)
$$

Em particular, com essas distribuições de equilíbrio obtemos o número médio e o tempo médio de espera de um cliente no sistema, como pode ser visto a seguir, levando-se em consideração a fórmula de Little $L=\lambda W$, onde $L$ é o número esperado de clientes no sistema e $W$, tempo médio de permanência do cliente no sistema. É importante salientar que conhecemos $L$, podemos obter $W$ aplicando-se a fórmula de Little e vice-versa.

Para o caso de $\lambda=5, \mu_{1}=\mu_{2}=6$, temos

$$
L=1,04997 \quad W=0,2100
$$


Para o caso de $\lambda=10,8, \mu_{1}=\mu_{2}=6$, temos

$$
L=4,3324 \quad W=0,4011
$$

\subsection{Considerações Finais}

Neste capítulo calculamos a distribuição conjunta de equilíbrio para o sisterna de filas emn parạlelo. Calculamos também a distribuiçāo do número total de clientes no sistema. A partir desses resultados, considerando-se uma fila finita, obtivemos o resultado numérico para o número médio de clientes no sistema e o tempo médio de permanência de um cliente no sistema. No próximo capítulo apresentamos uma nova proposta, no qual o modelo também é sistema de filas em paralelo, onde o serviço geral é especial, implicando que juntar-se à fila mais longa é mais benéfico. 


\section{Capítulo 3}

\section{Filas Paralelas com Serviços Dicotômicos}

\subsection{Introdução}

Neste capítulo apresentamos um modelo de duas filas em paralelo, abordado por Whitt(86) em seu artigo Deciding which Queue to Join: Some Counterexamples. O modelo proposto por Whitt é um sistema de filas $M /(G / 1)^{2}$, onde os clientes que chegam ao sistema conhecem somente os comprimentos das filas no momento da chegada. O processo de atendimento tem uma distribuição geral, onde o servidor atende por um tempo igual a zero, com probabilidade $1-\epsilon$ ou um serviço de comprimento igual a $n$, com probabilidade $\epsilon$, onde $\epsilon$ é um número muito pequeno.

Os clientes que chegam ao sistema selecionam a fila mais longa, se os comprimentos entre as filas for maior do que um.

Na Seção 3.2 apresentamos a caracterização do sistema de filas e o modo como os clientes juntam-se as elas.

Na seção 3.3 apresentamos os resultados de Whitt e, finalmente, na Seção 3.4, as considerações finais. 


\subsection{Característica do Sistema Proposto}

O modelo estudado por Whitt(86) é o sistema de uma fila $M /(G / 1)^{2}$ que apresenta as seguintes características: 2 servidores, cada um com sua própria fila; os dois servidores atendem os clientes com tempo de serviço distribuído de um modo geral i.i.d.; as salas de espera são ilimitadas; com disciplina FCFS, os clientes são servidos na ordem de chegada - não consideramos outros tipos de prioridades e nem há desistências dos clientes no sistema; os tempos entre as chegadas formam uma seqüência de variáveis aleatórias i.i.d. e com distribuição exponencial. Os tempos entre as chegadas e os tempos de serviços de cada um dos servidores são independentes.

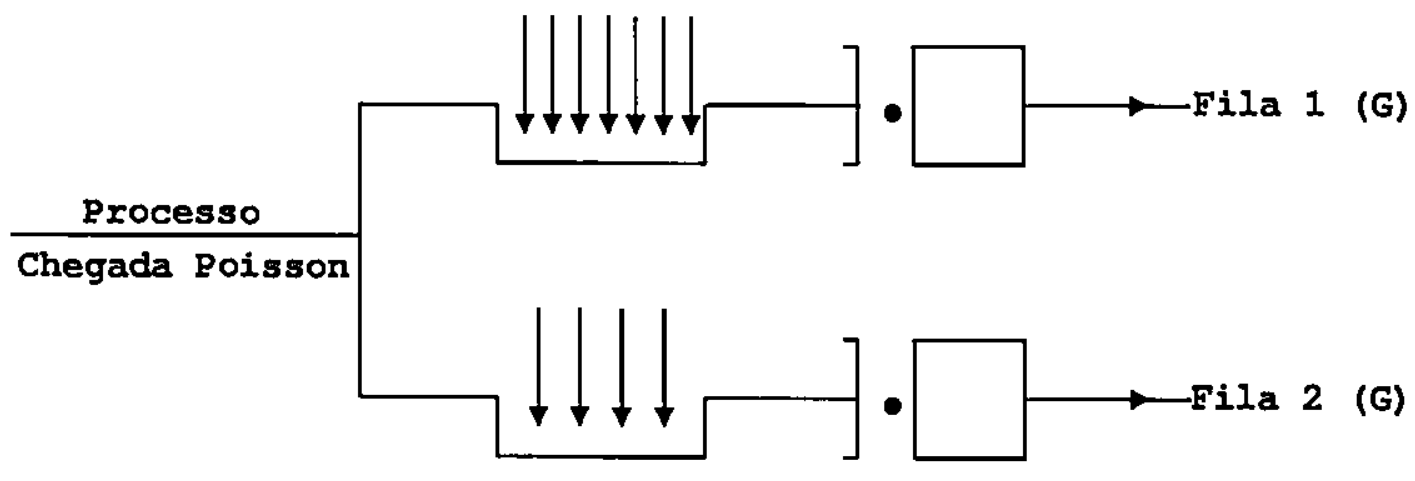

Figura 3.1: Uma representação do modelo $M /(G / 1)^{2}$

Neste capítulo avaliamos o tempo médio de espera em equilíbrio por cliente. Assim, utilizamos para calcular o tempo médio de espera em equilíbrio a estrutura regenerativa em um ciclo ocupado típico. Para isso definiremos os conceitos de processo regenerativo e um ciclo ocupado posteriormente.

A distribuição do tempo de serviço assume dois valores: serviço de comprimento zero com probabilidade $1-\epsilon$ e $n$ com probabilidade $\epsilon$. Com probabilidade alta ( $\epsilon$ tendendo a zero), os clientes que estão esperando na fila 1 devem sair no mesmo instante do cliente que termina o serviço nesta fila. 
O cliente que acaba de chegar ao sistema observa os comprimentos entre as filas e toma uma decisão de acordo com os critérios abaixo:

- se ambas as filas apresentam o mesmo comprimento, o cliente une-se a qualquer uma delas aleatoriamente;

- se a diferença entre o comprimento das filas for igual a um, o cliente une-se à fila que apresenta o comprimento menor;

- se a diferença entre os comprimentos das filas for maior ou igual a 2, o cliente une-se à fila que apresenta o comprimento maior quando nenhuma delas estiver vazia.

Neste modelo proposto por Whitt(89), o critério de juntar-se à fila mais curta nem sempre é a melhor escolha. De fato, com probabilidade muita alta, os clientes que estāo esperando na fila com comprimento de serviço zero saem no mesmo instante, quando acontece um fim de serviço nesta fila. O cliente que entra no sistema vê a diferença entre os comprimentos das filas maior do que um, seleciona a fila que apresenta o comprimento maior. Desta forma, com este tempo de serviço e este critério de escolha da fila, o cliente provavelmente terá um tempo de espera menor no sistema.

Consideremos o sistema vazio, os clientes que entram no sistema, com probabilidade alta $1-\epsilon$ saem imediatamente após a sua chegada, até que ambos os servidores estejam ocupados. A partir deste instante, os comprimentos das filas começarão a crescer; a próxima chegada selecionará a fila à qual irá juntar-se de acordo com os critérios já estabelecidos anteriormente.

\subsection{Resultados}

Os resultados de Whitt foram construídos utilizando-se a análise do tráfego leve no processo regenerativo definido em um ciclo ocupado. Daremos inicialmente as seguintes definições básicas: 
Definição 3.3.1. Processo estocástico $\{B(t): t \geq 0\}$, é dito regenerativo se existe uma variável aleatória $Y$ não negativa, tal que para todo instante $\dot{s} \geq 0$, satisfaz as seguintes condições:

(i) $\{B(t+Y): t \geq 0\}$ é independente de $\{B(t): t \leq s \quad Y>s\}$;

(ii) $\{B(t+Y): t \geq 0\}$ é equivalente estocasticamente a $\{B(t): t \geq 0\}$.

Para maiores esclarecimentos veja os livros de Wolff (1989, pág. 89) e Çinlar (1975, pág. 293).

Definiçāo 3.3.2. Um ciclo ocupado é o intervalo de tempo decorrido entre duas chegadas sucessivas que encontram o sistema completamente vazio.

Indicamos nas Figuras 3.2, 3.3 e 3.4 algumas realizações do modelo.

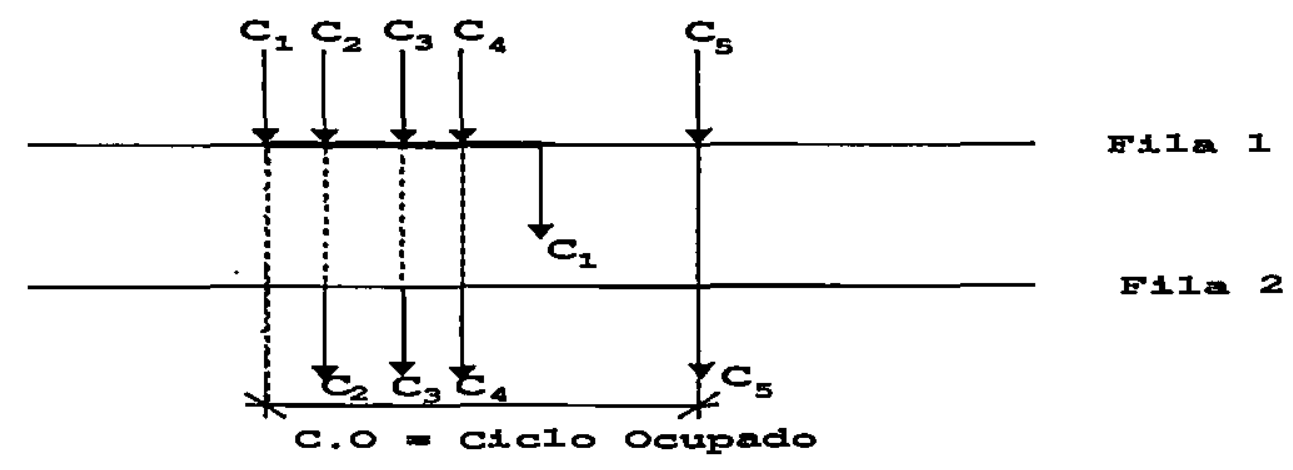

Figura 3.2: Realização de um ciclo ocupado com um serviço diferente de zero

Consideramos as chegadas dos clientes $C_{1}, C_{2}, \ldots, C_{5}$ na Figura 3.2. O ciclo ocupado é caracterizado pela chegada dos clientes $C_{1} e C_{5}$ que encontram o sistema completamente vazio. Os clientes $C_{2}, C_{3}, C_{4}$, por terem tempo de serviço igual a zero, não permanecem no sistema. 


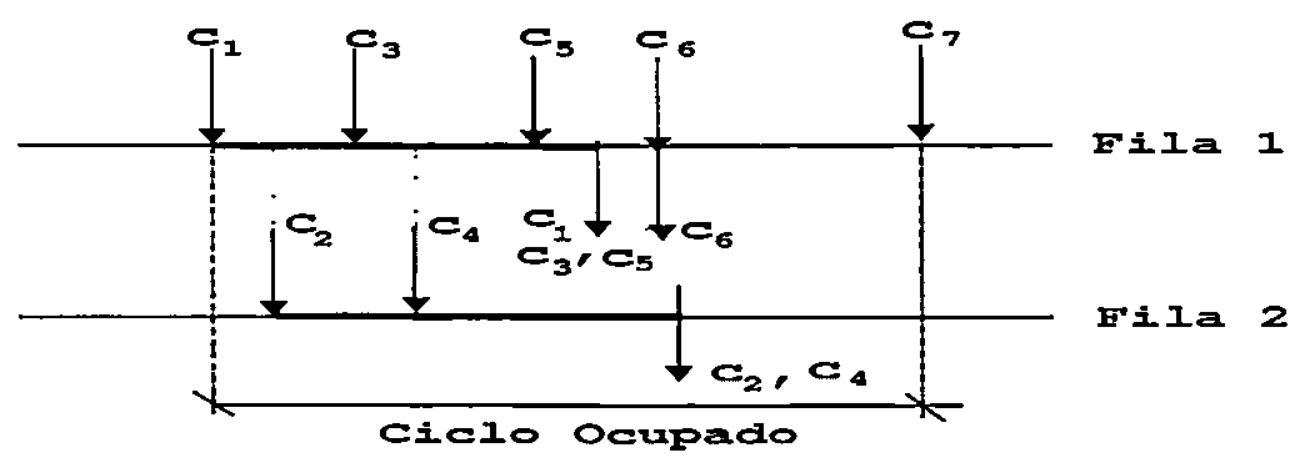

Figura 3.3: Realização de um ciclo ocupado com serviço nos dois servidores

No exemplo da Figura 3.3, consideramos 7 clientes passando pelo sistema. Os instantes de chegada dos clientes $C_{1}$ e $C_{7}$ definem um ciclo ocupado, enquanto os clientes $C_{1}, C_{3}$ e $C_{5}$ só deixam o sistema quando acabar o tempo de serviço do cliente $C_{1}$, por terem tempos de serviços iguais a zero. $O$ cliente $C_{6}$, por ter tempo de serviço igual a zero, sai direto do sistema, pois junta-se à fila 1 que está vazia. $O$ cliente $C_{4}$ sai do sistema junto com cliente $C_{2}$, pois apresenta tempo de serviço igual a zero.

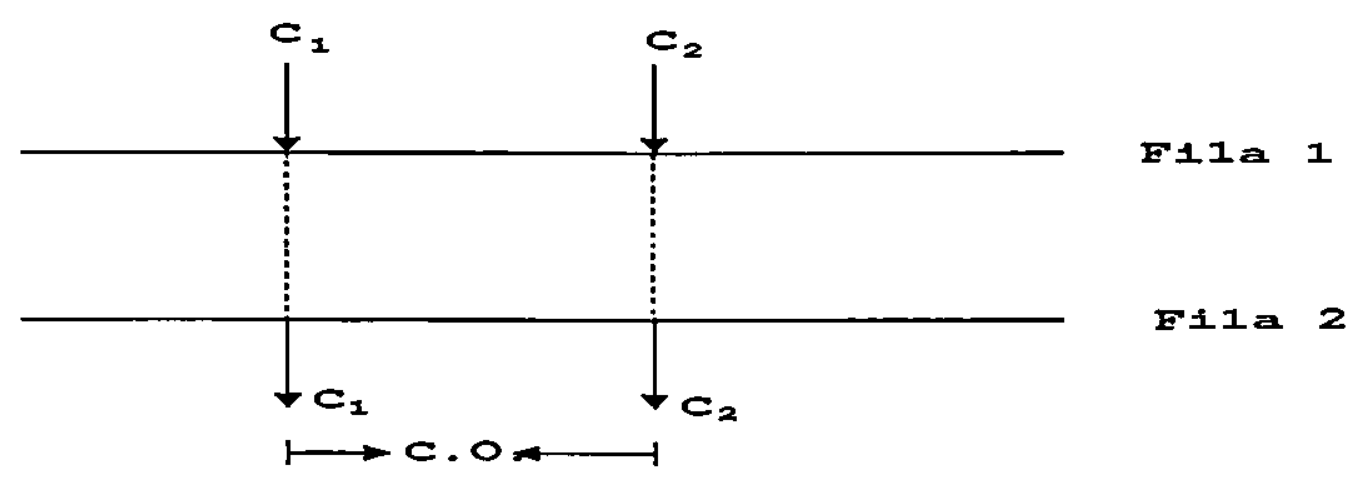

Figura 3.4: Realização de um ciclo ocupado sem serviço diferente de zero

$\mathrm{Na}$ Figura 3.4 temos o caso mais simples de ciclo ocupado. Os clientes $C_{1} e C_{2}$, com tempo de serviço igual a zero, ao entrarem no sistema partem imediatamente.

Iremos argumentar, atentando apenas para os serviços dos clientes, que a probabilidade de que $\mathrm{k}$ ou mais clientes tenham tempos de serviços diferentes de zero em um ciclo ocupado é da ordem de $\epsilon^{k}$. 
Consideremos $m$ chegadas independentes no sistema $M /(G / 1)^{2}$. As chegadas formam uma sucessão aleatória independente na qual se observa a realização de determinados serviços de comprimentos $n$ ou zero. O tempo de serviço de comprimento zero ocorre com probabilidade $1-\epsilon$ e o tempo de serviço de comprimento $n$ ocorre com probabilidade $\epsilon$. Representamos a variável aleatória $X$, a quantidade de clientes com serviço de comprimento $n$, para os $m$ clientes observados e assumimos que esse modelo seja binomial. A probabilidade de obter exatamente $k$ clientes com serviços de comprimento $n$ em um ciclo ocupado é dada por:

$$
P(X=k)=\left(\begin{array}{l}
m \\
k
\end{array}\right) \epsilon^{k}(1-\epsilon)^{m-k} \quad \text { onde } \quad k=0,1, \ldots, m
$$

Tomando-se $k=1$, temos: $P(X=1)=m \epsilon(1-\epsilon)^{m-1}$;

como $\epsilon \rightarrow 0$, logo $(1-\epsilon)^{m-1} \rightarrow 1$

Assim, $P(X=1) \cong \epsilon$

Para $k=2$, temos $P(X=2) \cong \epsilon^{2}$,

Generalizando-se para $k$ clientes temos

$$
P(X=k) \cong \epsilon^{k}
$$

De fato, conclui-se que a probabilidade de $\mathrm{k}$ clientes com tempo de serviço diferente de zero é da ordem de $\epsilon^{k}$, quando temos $m$ clientes no sistema.

Então, a probabilidade de alguma vez ter pelo menos um (dois) servidor(es) ocupado(s) em um ciclo ocupado é da ordem $\epsilon\left(\epsilon^{2}\right)$. Um cliente pode encontrar a diferença de pelo menos dois nos comprimentos das filas somente se há pelo menos três tempos de serviços diferentes de zero no ciclo ocupado, e a probabilidade é de ordem $\epsilon^{3}$. Ainda mais quando o cliente encontra tal diferença em um ciclo ocupado, deve unir-se à fila mais longa. 
A seguir faremos a demonstração formal deste resultado, considerando o ciclo regenerativo (ciclo ocupado).

Vamos utilizar o seguinte lema básico para mostrar o teorema que define limitantes para a variável aleatória condicionada que conta o número de clientes com tempo de serviço diferente de zero em ciclo ocupado.

Lema: Consideremos $X$ uma variável aleatória discreta que tem distribuição de Poisson, com média $\lambda$. Para todo número inteiro positivo $k$,

$$
\frac{\lambda^{k} e^{-\lambda}}{k !} \leq P(X \geq k) \leq \lambda^{k}
$$

Demonstração: Observe que $P(X=k) \leq P(X \geq k)$, para todo $k$. Portanto, o limite inferior é

$$
P(X=k)=\frac{\lambda^{k} e^{-\lambda}}{k !}
$$

E o limite superior é obtido por

$$
\begin{aligned}
P(X \geq k) & =\sum_{j=k}^{\infty} \frac{\lambda^{k} e^{-\lambda}}{k !}=\lambda^{k} \sum_{j=0}^{\infty} \frac{\lambda^{j} e^{-\lambda}}{(j+k) !} \\
& \leq \lambda^{k} \sum_{j=0}^{\infty} \frac{\lambda^{j} e^{-\lambda}}{j !}=\lambda^{k} .
\end{aligned}
$$

Assim, concluímos a prova do lema.

Consideremos os seguintes processos:

$A(t)$ : conta o número de chegadas no intervalo $[0, t]$, para $t \geq 0$

$N(t)$ : conta o número de chegadas no intervalo $[0, t]$, que tem tempo de serviço diferente de zero, ou melhor, tempo de serviço com comprimento $\mathrm{n}$.

As seguintes variáveis aleatórias condicionadas: 
$C$ : número de clientes atendidos em um ciclo ocupado;

$B$ : número de clientes com serviço diferente de zero em um ciclo ocupado;

$D$ : o evento em que o primeiro cliente no ciclo ocupado tenha tempo de serviço diferente de zero.

Utilizando-se o processo de chegada com $A(t)$, por uma Bernoulli que divide com probabilidade $\epsilon$ de ter serviço de comprimento $n$, e a probabilidade $1-\epsilon$ correspondendo ao serviço de comprimento zero, temos que $N(t)$ é um processo de Poisson com taxa $\lambda \epsilon$.

Teorema 3.3.1. Para qualquer inteiro positivo $k$, existem constantes positivas $M_{1}$ e $M_{2}$ tal que

$$
M_{1} \epsilon^{k} \leq P(B \geq k) \leq M_{2} \epsilon^{k}
$$

para todo $\epsilon \in(0,1)$.

Demonstração: Seja D o evento em que o primeiro cliente no ciclo ocupado tenha tempo de serviço diferente de zero.

Tomando-se $k \geq 1$, temos

$$
P(B \geq k)=P(B \geq k, D)+P(B \geq k, \bar{D}) \text {. }
$$

Mas $P(B \geq k, \vec{D})=0$, pois é a probabilidade de pelo menos $k$ ou mais clientes com tempo de serviço diferente de zero ocorrendo dentro de um ciclo ocupado, sendo que o primeiro cliente tem tempo de serviço igual a zero.

Entāo

$$
P(B \geq k)=P(B \geq k, D)=P(D) P(B \geq k \mid D)=\epsilon P(B \geq k \mid D),
$$

onde $P(D)=\epsilon$ : é a probabilidade do primeiro cliente com tempo de serviço diferente de zero em um ciclo ocupado. 
Mas

$$
P(N(n) \geq k-1) \leq P(B \geq k \mid D) \leq P(N(n(k-1)) \geq k-1)
$$

Em outras palavras, queremos dizer que, se temos $k-1$ ou mais tempos de serviço diferentes de zero no intervalo $[0, n]$, então certamente $B \geq k$, dado que o primeiro cliente tenha um tempo de serviço diferente de zero. Ainda, se $B \geq k$, então claramente devem existir pelo menos $k-1$ tempos de serviços diferentes de zero no intervalo $[0,(k-1) n]$. Aplicando-se o lema anterior, temos

$$
P(N(n(k-1)) \geq k-1) \leq(\lambda \epsilon n(k-1))^{k-1}
$$

e

$$
P(N(n) \geq k-1) \geq \frac{(n \epsilon \lambda)^{k-1}\left(e^{-\lambda \epsilon n}\right)}{(k-1) !}
$$

Tomando-se

$$
M_{1}=\frac{(\lambda n)^{k-1} e^{-\lambda n}}{(k-1) !} \quad \text { e } \quad M_{2}=(\lambda n(k-1))^{k-1}
$$

o teorema fica provado.

Vamos agora calcular o tempo médio de permanência do cliente no sistema em um ciclo ocupado. Seja $N$ o número de chegadas durante $[0, T]$, num ciclo ocupado, e $W_{1}, W_{2}, \ldots W_{k}$ os tempos de espera para os clientes $C_{1}, C_{2}, \ldots, C_{k}$ no sistema durante $[0, T]$. Então o tempo médio de espera em equilíbrio por cliente é obtido dividindo-se o tempo médio esperado pelos clientes e número esperado de clientes servidos em um ciclo ocupado, e dado por:

$$
E W=E\left(\sum_{k=1}^{C} \frac{W_{k}}{E C}\right)
$$

Onde

$W_{1}$ : o tempo de espera do cliente inicial que encontra o sistema vazio; 
$W_{k}$ : o tempo de espera do $k$-ésimo cliente atendido em um ciclo ocupado.

O seguinte teorema mostra como calcular o tempo médio esperado em equilíbrio para cada cliente em um processo regenerativo.

Teorema 3.3.2. Seja $C$ uma variável aleatória discreta que conta o número de clientes atendidos em ciclo ocupado, definida em um espaço probabilidade $(\Omega, \mathbf{A}, P)$, onde $E C$ e $E\left[\sum_{k=1}^{C} W_{k}\right]$ são dados por:

$$
E C=1+c_{1} \epsilon+c_{2} \epsilon^{2}+o\left(\epsilon^{2}\right)
$$

onde as constantes $c_{1}$ e $c_{2}$ são independentes do critério $e$

$$
E\left[\sum_{k=1}^{c} W_{k}\right]=b_{2} \epsilon^{2}+b_{3} \epsilon^{3}+o\left(\epsilon^{3}\right),
$$

onde a constante, $b_{2}$ é independente do critério, enquanto $b_{3}$ não é.

Então, o tempo médio esperado em equilibrio por cliente é obtido por:

$$
\begin{aligned}
E W & =\frac{b_{2} \epsilon^{2}+b_{3} \epsilon^{3}+o\left(\epsilon^{3}\right)}{1+c_{1} \epsilon+c_{2} \epsilon^{2}+o\left(\epsilon^{2}\right)} \\
& =b_{2} \epsilon^{2}+\left(b_{3}-c_{1} b_{2}\right) \epsilon^{3}+o\left(\epsilon^{4}\right)
\end{aligned}
$$

\subsection{Considerações Finais}

Neste capítulo mostramos que em um ciclo ocupado a probabilidade de ter pelo menos um (dois) servidor(es) ocupado(s) é da ordem de $\epsilon\left(\epsilon^{2}\right)$. Para $\epsilon$ pequeno, a diferença dos comprimentos entre as filas for suficientemente grande, o critério de unir-se à fila mais curta é melhor. Se a diferença dos comprimentos entre as filas não for muito grande e maior ou igual a dois, temos que o critério de unir-se à fila que apresenta o comprimento maior apresenta a melhor performance. No próximo capítulo apresentamos um sistema de filas em paralelo, porém o cliente esperto utiliza o critério de esperar e juntar-se à fila que produz o primeiro fim de serviço. 


\section{Capítulo 4}

\section{Observando Filas Paralelas com Serviços Exponenciais}

\subsection{Introdução}

O Capítulo 3 apresenta um exemplo específico de um sistema de filas paralelas, com uma distribuição especial para o tempo de serviço, no qual juntar-se à fila mais curta nem sempre é a melhor escolha. Neste sentido apresentamos agora uma nova proposta de observar filas paralelas de serviços exponenciais heterogêneos antes de juntar-se a elas. Apresentamos na Seção 4.2 o modelo e a notação do sistema de duas filas paralelas proposta no artigo de Hlynka, Stanford, Poon e Wang (94), Observing Queues before Joining, cujo objetivo é demonstrar que o tempo médio de permanência no sistema para um cliente esperto que utiliza a estratégia de observar filas paralelas antes de juntar-se a elas é melhor. Nem sempre esse tempo médio de permanência no sistema será o menor. Durante o tempo de observação do cliente esperto, ele pode demorar a juntar-se a uma fila até que algumas chegadas e uma conclusão de serviço tenham sido observadas. Todos os outros clientes que chegam e ficam à frente do cliente esperto seguem a estratégia de juntar-se à fila mais curta. $O$ cliente esperto tem então uma única opção: esperar a primeira conclusāo de serviço ocorrer e escolher a fila na qual aconteceu a conclusão. Portanto, este é o custo que o cliente esperto deverá pagar por retardar a sua decisão.

Este tipo de situação é de considerável aplicação em muitas áreas, como, por exemplo, na tecnologia de computadores com multiprocessadores, onde uma tarefa considerada 
importante deve esperar mais informaçōes sobre a velocidade dos processadores, antes de se juntar a um deles. Um outro exemplo, na indústria da construção, um cliente pode observar o desempenho de duas empresas prestadoras de serviço antes de fazer uma escolha. Na Seção (4.3) apresentamos as análises das estratégias, e na Seção 4.4 as tabelas comparativas do tempo médio de permanência do cliente esperto no sistema. Na Seção 4.5 finalmente apresentamos as considerações finais.

\subsection{Modelo}

Nesta seção descrevemos o sistema de fila $G /\left(\left(M_{1}+M_{2}\right) / 1\right)^{2}$, que apresenta as seguintes características: 2 servidores cada um com sua própria fila, os dois servidores atendem os clientes com tempo de serviço distribuído exponencialmente i.i.d. e com taxas de atendimentos diferentes; as salas de espera são ilimitadas; os tempos entre as chegadas são variáveis aleatórias gerais i.i.d. Os tempos entre as chegadas e os tempos de serviço de cada um dos servidores são independentes.

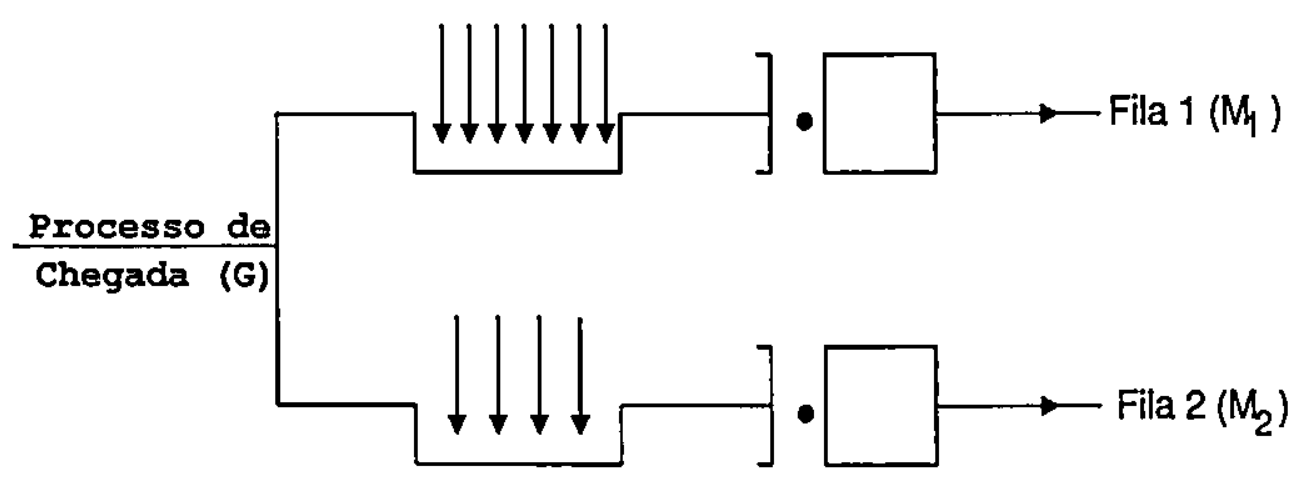

Figura 4.1: Uma representação do modelo $G /\left(\left(M_{1}+M_{2}\right) / 1\right)^{2}$

Este trabalho avalia o tempo médio que um cliente esperto permanece no sistema de fila $G /\left(\left(M_{1}+M_{2}\right) / 1\right)^{2}$ em equilíbrio. Para isso devemos analisar três estratégias de como o cliente esperto deve entrar no sistema. 


\subsection{Análises das Estratégias}

Vamos inicialmente descrever duas estratégias, sendo $S$ o cliente esperto.

Estratégia 1 Assumimos que $S$ se comporta como cliente regular, isto é, ele imediatamente junta-se à fila mais curta, se houver uma. Se ambas as filas têm o mesmo comprimento, $S$ imediatamente junta-se a uma das filas, com probabilidade $1 / 2$.

Estratégia 2 Se ambas as filas não estiverem vazias, então $S$ se comporta como na estratégia 1. Se ambas as filas estiverem vazias, $S$ observa algumas chegadas até que a primeira conclusão de serviço ocorra, então $S$ escolhe a fila na qual acabou de ocorrer o serviço.

Na seqüência introduzimos a notação, e demonstramos os teoremas que serão essenciais na análise destas estratégias.

- $A(t)$ : a função distribuição acumulada do tempo entre chegadas;

- $T$ : a variável aleatória dos tempos entre as chegadas;

- $E(T)$ : o tempo médio dos intervalos entre chegadas;

- TCSS : a variável aleatória que mede o tempo de permanência do cliente esperto no sistema;

- $E(T C S S$ | estratégia 1) : o tempo médio de permanência do cliente esperto sob a estratégia 1;

- $E(T C S S$ | estratégia 2) : o tempo médio de permanência do cliente esperto no sistema sob a estratégia 2 ;

- $\lambda$ : taxa de chegada no sistema;

- $\mu_{I}$ : taxa de atendimento do primeiro servidor; 
- $\mu_{2}$ : taxa de atendimento do segundo servidor.

Teorema 4.3.1. Sejam $A(t)$ e $E(T)$ definidos acima. Se $\mu_{1}>\lambda$ então

$$
\lim _{\mu_{2} \rightarrow 0} E(T C S S \mid \text { estratégia } 2) \leq \lim _{\mu_{2} \rightarrow 0} E(T C S S \mid \text { estratégia 1). }
$$

Demonstraçāo: Consideramos, para o sistema de filas $G /\left(\left(M_{1}+M_{2}\right) / 1\right)^{2}$, que ambas as filas não estejam vazias. Analisando-se o comportamento do cliente esperto sob a estratégia 1, o tempo médio de permanência do cliente esperto é dado por:

$$
\begin{gathered}
E(T C S S \mid \text { estratégia } 1)=\frac{1}{2 \mu_{1}}+\frac{1}{2 \mu_{2}} \\
\lim _{\mu_{2} \rightarrow 0} E(T C S S \mid \text { estratégia } 1)=\infty
\end{gathered}
$$

Consideremos agora que $S$ encontra o sistema vazio. Sob a estratégia 2 , ele observa um ou mais novos clientes se juntarem ao sistema e tomarem seus lugares na fila à sua frente. Então o cliente esperto escolhe a fila na qual ocorreu a primeira conclusão, conforme a figura abaixo.

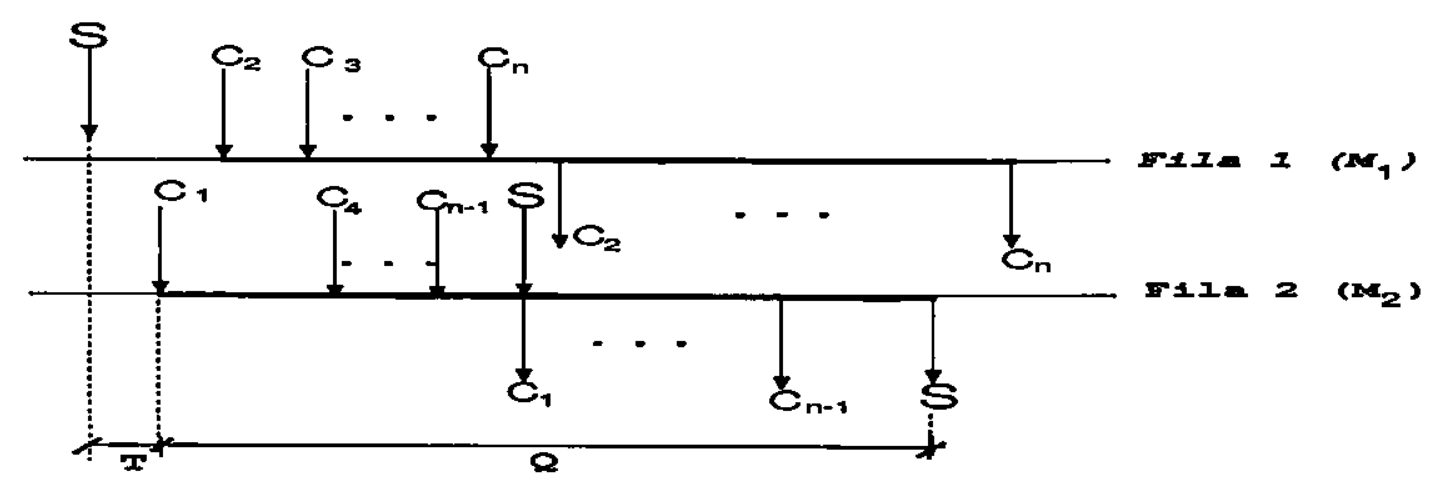

Figura 4.2: Uma representaçāo de uma realização do modelo

Neste caso o cliente esperto observa os novos clientes se juntarem ao sistema, de acordo com a estratégia 1. Considere a seguinte notação e o lema a seguir: 
$E[Q \mid l]$ : o tempo médio a partir do instante em que o primeiro cliente começa o serviço no sistema, até a conclusão de $S$, sabendo-se que o primeiro cliente escolhe aleatoriamente qualquer uma das filas $l=1,2$ com mesma probabilidade.

Lema 4.3.1. Sejam $E[T]$ e $E[Q \mid l], l=1,2$ definidos acima. Então o tempo médio que o cliente esperto permanece no sistema sob a estratégia 2 é dado por

$$
E(T C S S \mid \text { estratégia } 2)=E(T)+\frac{1}{2} \sum_{l=1}^{2} E(Q \mid l)
$$

Demonstração: Para demonstrarmos este lema vamos analisar dois casos possíveis onde o cliente esperto chega, encontra o sistema vazio e observa antes de juntar-se a uma das filas. No primeiro caso um cliente $C_{1}$ entra no sistema, é atendido pelo servidor 1 e termina o serviço antes da chegada do cliente $C_{2}$, portanto o cliente esperto espera a conclusão do serviço de $C_{1}$ e seleciona a fila na qual ocorreu essa conclusão, conforme figura abaixo.

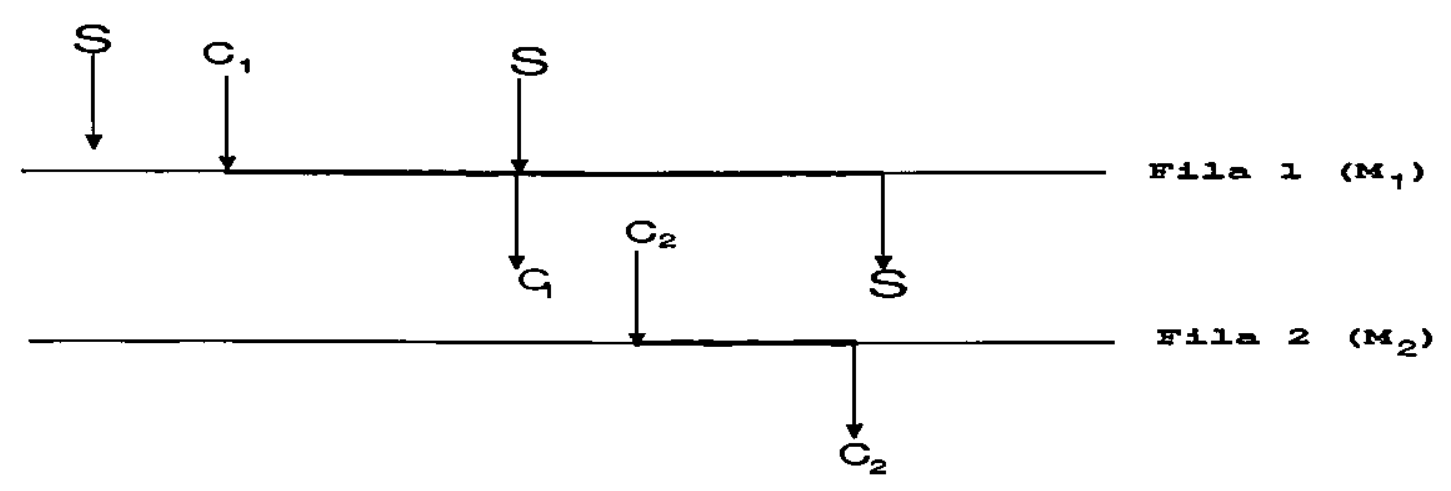

Figura 4.3: Uma representação de uma realização simples do modelo

No segundo caso, com o início do serviço de $C_{2}$, antes do término do serviço de $C_{1}$, ambos servidores tornam-se ocupados, portanto os novos clientes que chegam ao sistema escolhem uma das filas de acordo com a estratégia 1. Suponhamos que $C_{1}$ termine o serviço depois de $C_{2}$, então o cliente esperto escolhe a fila na qual ocorreu o primeiro fim de serviço, conforme a representação da Figura 4.4.

Utilizaremos a seguinte notação: 
$R$ : a variável aleatória que representa o tempo desde o início do serviço de $C_{1}$ até o próximo evento ocorrer, ou seja, uma chegada ou fim de serviço;

$Y$ : a variável aleatória que representa o tempo no sistema a partir do momento em que ambos servidores estejam ocupados até que $S$ complete o serviço;

$Q$ : a variável aleatória que representa o tempo desde o instante em que o cliente $C_{1}$ começa o serviço até que $S$ complete o serviço.

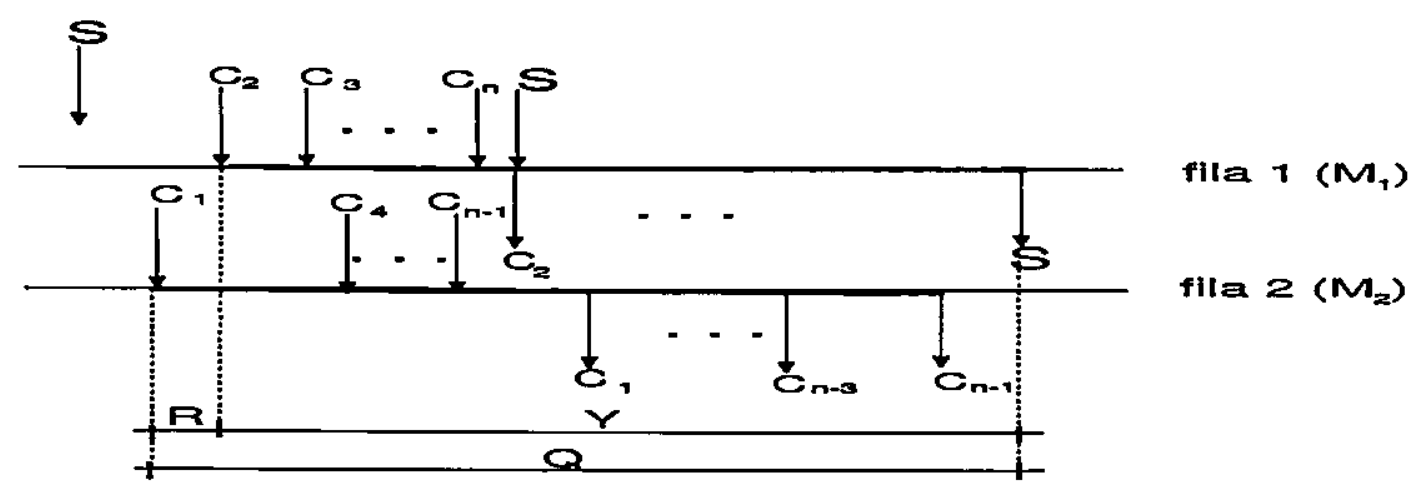

Figura 4.4: Uma representação de uma realizaçāo do modelo

Assim, podemos calcular o tempo médio de espera a partir do instante em que o cliente $C_{1}$ comece o serviço até que $S$ conclua o serviço. Temos:

$$
E(Q \mid l)=E(R \mid l)+\left(1-P\left(A_{2} \mid l\right)\right)\left(\frac{1}{\mu_{l}}\right)+P\left(A_{2} \mid l\right) E(Y)
$$

A probabilidade $P\left(A_{2} \mid l\right)$ do evento de ocorrer uma chegada após o inf́cio de serviço de $C_{1}$, na fila $l$, caracterizada na figura 4.4, é a mesma que a probabilidade que o servidor $l$ não termine o serviço durante o intervalo de tempo até a próxima chegada, que é igual a

$$
P\left(A_{2} \mid l\right)=\int_{0}^{\infty} e^{-\mu_{l} t} d A(t)=\phi\left(\mu_{l}\right)
$$


O tempo médio até o próximo evento ocorrer é o mínimo entre as duas variáveis aleatórias: o intervalo de tempo até a próxima chegada e o fim de serviço de $C_{2}$. Assim, obtemos

$$
\begin{aligned}
E(R \mid l) & =\int_{0}^{\infty} P(R>t \mid l) d t=\int_{0}^{\infty}(1-A(t)) e^{-\mu_{l} t} d t= \\
& =\int_{0}^{\infty} e^{-\mu_{l} t} d t-\int_{0}^{\infty} A(t) e^{-\mu_{l} t} d t= \\
& =-\left.\frac{1}{\mu_{l}} e^{-\mu_{l} t}\right|_{0} ^{\infty}+\frac{1}{\mu_{l}} \int_{0}^{\infty} A(t) d\left(e^{-\mu_{l} t}\right),
\end{aligned}
$$

aplicando a fórmula de integração por partes das integrais de Lebesgue-Stieltjes,

$$
\int_{a}^{b} g d f=f(b) g(b)-f(a) g(a)-\int_{a}^{b} f d g
$$

obtemos

$$
E(R \mid l)=\frac{1}{\mu_{l}}-\frac{1}{\mu_{l}} \phi\left(\mu_{l}\right)=\frac{1}{\mu_{l}}\left(1-\phi\left(\mu_{l}\right)\right)
$$

Portanto,

$$
E(R \mid l)=\frac{1}{\mu_{l}}\left(1-\phi\left(\mu_{l}\right)\right)
$$

O tempo médio de espera do cliente esperto, a partir do instante em que ambos os servidores estejam ocupados até que $S$ complete o serviço é obtido da seguinte forma: o cliente esperto espera em média $\frac{1}{\mu_{1}+\mu_{2}}$ para que um dos dois clientes $C_{1}$ ou $C_{2}$ conclua o serviço. Assim, a probabilidade do cliente $C_{j}, j \in l=1,2$, terminar o serviço é igual a $\frac{\mu_{j}}{\mu_{1}+\mu_{2}}$.

Suponhamos que $N$ seja o número total de clientes que chegam ao sistema no intervalo de tempo a partir do instante em que os servidores estejam ocupados até o primeiro fim 
de serviço. Logo, $S$ espera em média $\frac{E(N)}{2}$ para ser atendido. Assim temos:

$$
\begin{aligned}
E(Y) & =\frac{1}{\mu_{1}+\mu_{2}}+\sum_{l=1}^{2} \frac{\mu_{l}}{\mu_{1}+\mu_{2}}\left(\frac{E(N)}{2}+1\right)\left(\frac{1}{\mu_{l}}\right)= \\
& =\frac{1}{\mu_{1}+\mu_{2}}+\frac{1}{\mu_{1}+\mu_{2}}(E(N)+2) .
\end{aligned}
$$

Portanto,

$$
E(Y)=\frac{E(N)+3}{\mu_{1}+\mu_{2}} .
$$

Substituindo-se as equações (4.5) e (4.6) em (4.4), obtemos

$$
E(Q \mid l)=\frac{2}{\mu_{l}}\left(1-\phi\left(\mu_{l}\right)\right)+\frac{1}{2} \frac{\phi\left(\mu_{l}\right)}{\mu_{1}+\mu_{2}}(E(N)+3) .
$$

Substituindo-se a equação (4.7) na equação (4.3), obtemos

$$
E(T C S S \mid \text { estratégia } 2)=E(T)+\frac{1}{2} \sum_{l=1}^{2} E(Q \mid l),
$$

ou seja,

$$
E\left(T C S S \mid \text { estratégia 2) }=E(T)+\sum_{l=1}^{2}\left[\frac{1-\phi\left(\mu_{l}\right)}{\mu_{l}}+\frac{1}{2} \frac{\phi\left(\mu_{l}\right)}{\mu_{1}+\mu_{2}}(E(N)+3)\right]\right. \text {. }
$$

Logo,

$$
\begin{aligned}
\lim _{\mu_{2} \rightarrow 0} E(T C S S \mid \text { estratégia 2) = } & \lim _{\mu_{2} \rightarrow 0} E[T]+\lim _{\mu_{2} \rightarrow 0} \frac{1-\phi\left(\mu_{1}\right)}{\mu_{1}}+\lim _{\mu_{2} \rightarrow 0} \frac{1-\phi\left(\mu_{2}\right)}{\mu_{2}}+ \\
& \lim _{\mu_{2} \rightarrow 0} \frac{1}{2} \phi\left(\mu_{1}\right) E(Y)+\lim _{\mu_{2} \rightarrow 0} \frac{1}{2} \phi\left(\mu_{2}\right) E(Y)= \\
= & E[T]+\frac{1-\phi\left(\mu_{1}\right)}{\mu_{1}}+\frac{1}{2} \phi\left(\mu_{1}\right) E(Y)+ \\
& \frac{1}{2} E(Y)+\lim _{\mu_{2} \rightarrow 0} \frac{1-\phi\left(\mu_{2}\right)}{\mu_{2}}
\end{aligned}
$$


aplicando-se a regra de L'Hôpital obtemos

$$
\lim _{\mu_{2} \rightarrow 0} \frac{1-\phi\left(\mu_{2}\right)}{\mu_{2}}=\lim _{\mu_{2} \rightarrow 0} \frac{\lambda}{\left(\mu_{2}+\lambda\right)^{2}}=\frac{1}{\lambda}=E[T] .
$$

Logo,

$$
\lim _{\mu_{2} \rightarrow 0} E(T C S S \mid \text { estratégia } 2)=2 E[T]+\frac{1-\phi\left(\mu_{1}\right)}{\mu_{1}}+\frac{1}{2}\left(\phi\left(\mu_{1}\right)+1\right) E(Y) .
$$

Portanto, para a estratégia 1 o tempo médio de permanência do cliente esperto no sistema tende para o infinito e na estratégia 2 tende ao um valor finito. Concluímos assim a demonstração do Teorema 4.3.1.

Vamos calcular agora o número médio, $N=n$, de clientes que entram no sistema, a partir do instante em que ambos os servidores estejam ocupados até a primeira conclusão de serviço. Temos $N=n$ intervalos entre as chegadas durante as quais nenhuma conclusão acontece. Sabemos que cada chegada ocorre independentemente com probabilidade $\phi\left(\mu_{1}+\mu_{2}\right)$, que é a probabilidade do mínimo entre o intervalo de tempo entre a próxima chegada e tempo para que um dos dois clientes $C_{1}$ ou $C_{2}$ conclua o serviço. Finalmente, a conclusão de serviço do cliente esperto durante os $n+1$ intervalos entre chegadas acontece com probabilidade $p=1-\phi\left(\mu_{1}+\mu_{2}\right)$. Podemos perceber que $N$ é uma variável aleatória discreta com distribuição geométrica defasada que consiste em contar o número de clientes que entram no sistema até a entrada do cliente esperto em uma das filas. Isso significa dizer que a última chegada é a do cliente esperto, que por sua vez não é computada. Assim, ela é definida a partir de $j=0$. Vamos calcular $E(N)$ que é dado por:

$$
E(N)=\frac{1}{p}-1=\frac{\phi\left(\mu_{1}+\mu_{2}\right)}{1-\phi\left(\mu_{1}+\mu_{2}\right)}
$$

Suponhamos agora o caso onde as chegadas sejam Poisson de taxa $\lambda$. A equação (4.8) se reduz a

$$
E(T C S S \mid \text { estratégia } 2)=\frac{1}{\lambda}+(1+\rho)\left(1+\frac{\rho}{2}\right) \sum_{l=1}^{2} \frac{1}{\mu_{l}+\lambda} .
$$


Vamos demonstrar a equação acima. Definimos

$$
\begin{array}{ll}
A(t)=1-e^{-\lambda t}, & \phi\left(\mu_{l}\right)=\int_{0}^{\infty} e^{-\mu_{l} t} \lambda e^{-\lambda t} d t=\frac{\lambda}{\mu_{l}+\lambda} \\
E(N) & =\frac{\lambda}{\mu_{1}+\mu_{2}}=\rho .
\end{array}
$$

Obtemos

$$
\phi\left(\mu_{1}\right)=\frac{\lambda}{\mu_{1}+\lambda}, \quad \phi\left(\mu_{2}\right)=\frac{\lambda}{\mu_{2}+\lambda}, \quad \phi\left(\mu_{1}+\mu_{2}\right)=\frac{\lambda}{\mu_{1}+\mu_{2}+\lambda} .
$$

Substituindo-se os resultados acima na equação (4.8), obtemos

$$
\begin{gathered}
E\left(T C S S \mid \text { estratégia 2) }=E(T)+\sum_{l=1}^{2}\left[\frac{1-\phi\left(\mu_{l}\right)}{\mu_{l}}+\frac{1}{2} \frac{\phi\left(\mu_{l}\right)}{\mu_{1}+\mu_{2}}(E(N)+3)\right]=\right. \\
=\frac{1}{\lambda}+\frac{1-\phi\left(\mu_{1}\right)}{\mu_{1}}+\frac{1-\phi\left(\mu_{2}\right)}{\mu_{2}}+\frac{\rho}{2}\left(\frac{3+\rho}{\mu_{1}+\mu_{2}}\right) \frac{\lambda}{\mu_{1}+\lambda}+\frac{\rho}{2}\left(\frac{3+\rho}{\mu_{1}+\mu_{2}}\right) \frac{\lambda}{\mu_{2}+\lambda}= \\
=\frac{1}{\lambda}+\frac{1-\frac{\lambda}{\mu_{1}+\lambda}}{\mu_{1}}+\frac{1-\frac{\lambda}{\mu_{2}+\lambda}}{\mu_{2}}+\frac{\rho}{2}(3+\rho) \frac{\lambda}{\mu_{1}+\mu_{2}}\left(\frac{1}{\mu_{1}+\lambda}+\frac{1}{\mu_{2}+\lambda}\right)= \\
=\frac{1}{\lambda}+\sum_{l=1}^{2} \frac{1}{\mu_{l}+\lambda}+\frac{\rho}{2}(3+\rho) \sum_{l=1}^{2} \frac{1}{\mu_{l}+\lambda} \\
E(T C S S \mid \text { estratégia } 2)=\frac{1}{\lambda}+(1+\rho)\left(1+\frac{\rho}{2}\right) \sum_{l=1}^{2} \frac{1}{\mu_{l}+\lambda} \\
E(T C S S \mid \text { estratégia } 2)=\frac{1}{\lambda}+\left[1+\frac{\rho}{2}(2+1+\rho)\right] \sum_{l=1}^{2} \frac{1}{\mu_{l}+\lambda}=
\end{gathered}
$$


concluindo-se assim a demonstração da equação (4.11).

Vamos analisar agora as duas estratégias quando uma das taxas tende ao infinito. Para isso precisamos do seguinte teorema.

Teorema 4.3.2. Assumindo as mesmas estratégias, distribuições e definições do Teorema 4.3.1, suponhamos agora que $\lambda>\mu_{2}$. Então,

$$
\lim _{\mu_{1} \rightarrow \infty} E\left(T C S S \mid \text { estratégia 2) } \leq \lim _{\mu_{1} \rightarrow \infty} E(T C S S \mid \text { estratégia 1) }\right.
$$

se, e somente se,

$$
\phi\left(\mu_{2}\right)-\mu_{2} E(T) \geq \frac{1}{2}
$$

Demonstração: Vamos primeiramente determinar o tempo médio sob a estratégia 2 , considerando a taxa média $\mu_{1} \rightarrow \infty$. Conforme o resultado (4.9),

$$
\begin{aligned}
& \lim _{\mu_{1} \rightarrow \infty} E\left(T C S S \mid \text { estratégia 2) }=\lim _{\mu_{1} \rightarrow \infty}\left[E(T)+\sum_{l=1}^{2}\left[\frac{1-\phi\left(\mu_{l}\right)}{\mu_{l}}+\frac{1}{2} \frac{\phi\left(\mu_{l}\right)}{\mu_{1}+\mu_{2}}(E(N)+3)\right]\right]=\right. \\
& =E(T)+\lim _{\mu_{1} \rightarrow \infty} \frac{1-\phi\left(\mu_{1}\right)}{\mu_{1}}+\lim _{\mu_{1} \rightarrow \infty} \frac{1-\phi\left(\mu_{2}\right)}{\mu_{2}}+\lim _{\mu_{1} \rightarrow \infty} \frac{1}{2} \frac{\phi\left(\mu_{1}\right)}{\mu_{1}+\mu_{2}}(E(N)+3) \\
& \quad+\lim _{\mu_{1} \rightarrow \infty} \frac{1}{2} \frac{\phi\left(\mu_{2}\right)}{\mu_{1}+\mu_{2}}(E(N)+3) .
\end{aligned}
$$

Logo,

$$
\lim _{\mu_{1} \rightarrow \infty} E\left(T C S S \mid \text { estratégia 2) }=E(T)+\frac{1-\phi\left(\mu_{2}\right)}{\mu_{2}}\right. \text {. }
$$

O tempo médio do cliente esperto sob a estratégia 1 quando a taxa média de serviço do primeiro servidor $\mu_{1} \rightarrow \infty$ é igual a

$$
\lim _{\mu_{1} \rightarrow \infty} E\left(T C S S \mid \text { estratégia 1) }=\lim _{\mu_{1} \rightarrow \infty}\left(\frac{1}{2 \mu_{1}}+\frac{1}{2 \mu_{2}}\right)=\frac{1}{2 \mu_{2}}\right.
$$


Comparando-se as duas estratégias temos

$$
\begin{aligned}
\lim _{\mu_{1} \rightarrow \infty} E(T C S S \mid \text { estratégia 2) } & \leq \lim _{\mu_{1} \rightarrow \infty} E(T C S S \mid \text { estratégia 1), } \\
E(T)+\frac{1-\phi\left(\mu_{2}\right)}{\mu_{2}} & \leq \frac{1}{2 \mu_{2}} \\
\phi\left(\mu_{2}\right)-\mu_{2} E(T) & \geq \frac{1}{2}
\end{aligned}
$$

o que conclui a prova do Teorema 4.3.2.

Lema 4.3.2. Seja $\phi\left(\mu_{2}\right)-\mu_{2} E(T) \geq \frac{1}{2}$ satisfazendo o Teorema 4.3.2. Para o processo de chegada Poisson com taxa média igual a $\lambda$, temos

$$
\lambda>0.5(3+\sqrt{17}) \mu_{2} .
$$

Demonstraçāo: Usando a transformada de Laplace-Stieltjes da distribuição do tempo de serviço para o segundo servidor exponencial, a expressão

$$
\phi\left(\mu_{2}\right)-\mu_{2} E(T) \geq \frac{1}{2}
$$

torna-se

$$
\frac{\lambda}{\lambda+\mu_{2}}-\mu_{2} \frac{1}{\lambda} \geq \frac{1}{2},
$$

ou seja,

$$
\lambda^{2}-3 \mu_{2} \lambda-2 \mu_{2}^{2} \geq 0
$$

Esta última inequação tem uma solução positiva satisfazendo o teorema acima que é dada por

$$
\lambda>0.5(3+\sqrt{17}) \mu_{2},
$$

o que demonstra o Lema 4.3.2.

Vamos descrever agora a terceira estratégia e comparar com a estratégia 1. 
Estratégia 3 Consideremos $n_{l}$ o número de clientes na fila $l, l=1,2$. Se ambas as filas forem não vazias e $\left|n_{1}-n_{2}\right|>1$, então $S$ se junta à fila mais curta. Se ambas as filas forem não vazias e $\left|n_{1}-n_{2}\right| \leq 1$, então $S$ espera pela conclusão do primeiro serviço e se junta à fila na qual a conclusão do serviço aconteceu. Se exatamente uma das duas filas estiver vazia, então $S$ se junta à fila vazia. Se ambas as filas estiverem vazias, $S$ junta-se a uma delas com probabilidade $1 / 2$.

Enunciaremos alguns lemas e teoremas necessários para demonstrar o teorema (4.3.3).

Lema 4.3.3. Assumimos o sistema de fila $G /\left(\left(M_{1}+M_{2}\right) / 1\right)^{2}$ onde a fila 1 tem $i$ clientes e a fila 2 tem $j$ clientes com $|i-j| \leq 1$ para $i \geq 1, j \geq 1$. O cliente $S$ chega e espera pela primeira conclusão de serviço e junta-se a esta fila. O tempo médio de permanência do cliente esperto é igual a

$$
E(T C S S \mid \text { estratégia } 3)=\frac{1}{\mu_{1}+\mu_{2}}(E(N)+i+j+1) .
$$

Demonstração: Assumimos que $|i-j| \leq 1$. O cliente esperto espera o primeiro servidor completar o serviço, daí ele escolhe a fila na qual ocorreu um fim de serviço, compensando o cliente que acabou de sair. Assim, o comprimento da fila 1 é igual a $i+\frac{E(N)}{2}$ e o valor correspondente para a fila 2 é igual a $j+\frac{E(N)}{2}$, onde $E(N)$ é número médio de clientes que chegam a partir do instante em que ambos servidores estejam ocupados até que $S$ seleciona uma das filas. Suponhamos que o servidor $l$ termine primeiro o serviço com probabilidade $\frac{\mu_{l}}{\mu_{1}+\mu_{2}}$. Portanto, o tempo médio de permanência do cliente esperto é dado por:

$$
\begin{aligned}
E(T C S S \mid \text { estratégia 3) } & =\left[\mu_{1}\left(i+\frac{E(N)}{2}\right) \frac{1}{\mu_{1}}+\mu_{2}\left(j+\frac{E(N)}{2}\right) \frac{1}{\mu_{2}}\right] \frac{1}{\mu_{1}+\mu_{2}}+\frac{1}{\mu_{1}+\mu_{2}}= \\
& =\frac{1}{\mu_{1}+\mu_{2}}(E(N)+i+j+1) .
\end{aligned}
$$

Podemos então comparar o desempenho do cliente $S$ de acordo com as estratégias 1 e 3 . 
Teorema 4.3.3. Sejam a e b constantes positivas, tais que $a<\frac{\mu_{1}}{\mu_{2}}<b$. Fixemos $\lambda>0$, para $\mu_{1}$ (ou $\left.\mu_{2}\right)$ suficientemente grande. Então

$\{E(T C S S \mid$ estratégia 3) $-E(T C S S \mid$ estratégia 1$)\}<0$.

Para provarmos o Teorema 4.3.3 necessitamos de dois lemas adicionais. Assumimos que os intervalos entre as chegadas e os tempos de serviços são exponenciais. Seja $v_{i j}$ a probabilidade limite que o sistema se encontra no estado $(i, j)$, onde $i$ representa o número de clientes na fila 1 e $j$, o número de clientes na fila 2 . Os clientes que entraram no sistema obedeceram a estratégia 1. Enunciaremos a seguir o primeiro lema.

Lema 4.3.4. Seja $\lambda$ a taxa de chegada no sistema e $\mu_{1}$ e $\mu_{2}$, as taxas de serviço. Assumimos que $\mu_{1}, \mu_{2} \neq 0$, e $\lambda<\min \left(\mu_{1}, \mu_{2}\right)$. Então para $k \geq 2$

$$
\sum_{i+j=k} v_{i j} \leq\left(\frac{\lambda}{\min \left(\mu_{1}, \mu_{2}\right)}\right)^{k-2} \sum_{i=0}^{2} v_{i, 2-i}
$$

Demonstração: Fixemos k, apliquemos a equação global de balanço para cada estado tal que $i+j \leq k$. Tomando $\mathrm{k}=2$, temos o conjuntos de estados: $(0,2),(1,2) e(2,0)$ então

$$
\lambda v_{02}=\mu_{2} v_{03}+\mu_{1} v_{12}
$$

$$
\begin{aligned}
& \lambda v_{11}=\mu_{2} v_{12}+\mu_{1} v_{21}, \\
& \lambda v_{20}=\mu_{2} v_{21}+\mu_{1} v_{30}, \\
& \lambda\left(v_{02}+v_{11}+v_{20}\right)=\mu_{2} v_{03}+\mu_{1} v_{30}+\left(\mu_{1}+\mu_{2}\right)\left(v_{12}+v_{21}\right) \\
& o u \\
& \lambda \sum_{i+j=2} v_{i j}=\mu_{2} v_{03}+\mu_{1} v_{30}+\left(\mu_{1}+\mu_{2}\right) \sum_{i=1}^{k} v_{i, k+1-i}
\end{aligned}
$$

Para $k=3$, os conjuntos de estados possíveis são $(0,3),(1,2),(2,1)$ e $(3,0)$. Aplicando- 
se a equação global de balanço temos:

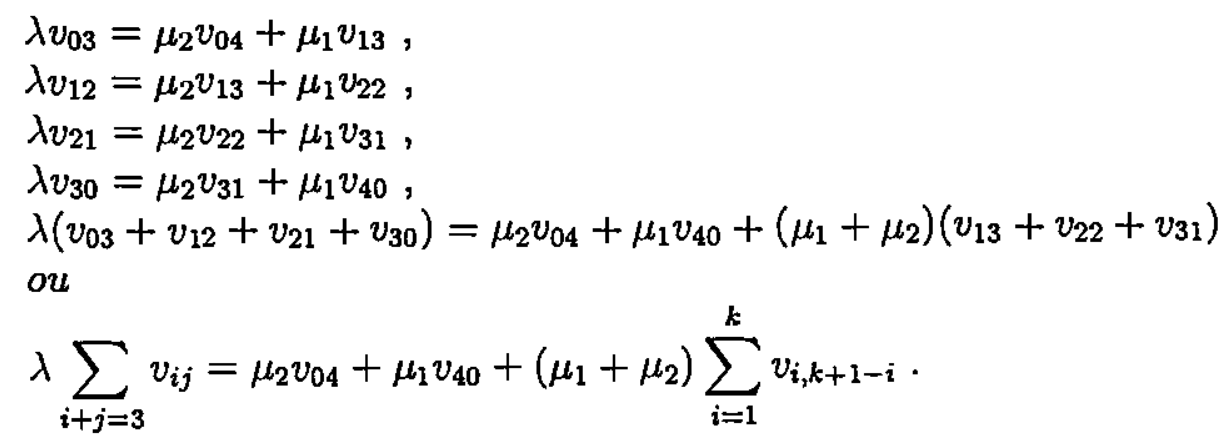

Generalizando-se para $k$ temos:

$$
\lambda \sum_{i+j=k} v_{i j}=\mu_{2} v_{0, k+1}+\mu_{1} v_{k+1,0}+\left(\mu_{1}+\mu_{2}\right) \sum_{i=1}^{k} v_{i, k+1-i}
$$

Como

$$
\left\{\begin{array}{l}
\min \left(\mu_{1}, \mu_{2}\right) \leq \mu_{1} \leq \max \left(\mu_{1}, \mu_{2}\right) \\
\min \left(\mu_{1}, \mu_{2}\right) \leq \mu_{2} \leq \max \left(\mu_{1}, \mu_{2}\right)
\end{array}\right.
$$

temos

$$
\begin{aligned}
& \lambda \sum_{i+j=k} v_{i j} \geq \min \left(\mu_{1}, \mu_{2}\right)\left[v_{0, k+1}+v_{k+1,0}+2 \sum_{i=0}^{k+1} v_{i, k+1-i}\right] \\
& \lambda \sum_{i+j=k} v_{i j} \geq 2 \min \left(\mu_{1}, \mu_{2}\right) \sum_{i=0}^{k+1} v_{i, k+1-i}, \\
& \sum_{i=0}^{k+1} v_{i, k+1-i} \leq \frac{1}{2}\left(\frac{\lambda}{\min \left(\mu_{1}, \mu_{2}\right)}\right) \sum_{i+j=k} v_{i j} \leq\left(\frac{\lambda}{\min \left(\mu_{1}, \mu_{2}\right)}\right) \sum_{i+j=k} v_{i j} .
\end{aligned}
$$

Utilizando-se esta desigualdade, vamos provar (4.13) por indução sobre $k$. O caso $k=2$ é imediato. Suponhamos que (4.13) seja válido para $k \geq 2$, isto é,

$$
\sum_{i+j=k} v_{i j} \leq\left(\frac{\lambda}{\min \left(\mu_{1}, \mu_{2}\right)}\right)^{k-2} \sum_{i=0}^{2} v_{i, 2-i}
$$

Para $k+1 \geq 2$, temos

$$
\sum_{i+j=k+1} v_{i j}=\sum_{i=0}^{k+1} v_{i, k+1-i} \leq\left(\frac{\lambda}{\min \left(\mu_{1}, \mu_{2}\right)}\right) \sum_{i+j=k} v_{i j} \leq\left(\frac{\lambda}{\min \left(\mu_{1}, \mu_{2}\right)}\right)\left(\frac{\lambda}{\min \left(\mu_{1}, \mu_{2}\right)}\right)^{k-2} \sum_{i=0}^{2} v_{i, 2-i} \leq
$$




$$
\leq\left(\frac{\lambda}{\min \left(\mu_{1}, \mu_{2}\right)}\right)^{(k+1)-2} \sum_{i=0}^{2} v_{i, 2-i}
$$

o que conclui a prova do lema.

Temos agora o segundo lema.

Lema 4.3.5. Seja a taxa de chegada no sistema $\lambda$ menor ou igual à soma das taxas de serviços nos dois servidores $e \mu_{1}, \mu_{2} \neq 0$. Então

$$
\left(v_{02}+v_{20}\right)\left(1-\left(\frac{\max \left(\mu_{1}, \mu_{2}\right)}{\lambda+\min \left(\mu_{1}, \mu_{2}\right)}\right)\left(\frac{\lambda}{\min \left(\mu_{1}, \mu_{2}\right)}\right)\right) \leq\left(\frac{\max \left(\mu_{1}, \mu_{2}\right)}{\lambda+\min \left(\mu_{1}, \mu_{2}\right)}\right)\left(\frac{\lambda}{\min \left(\mu_{1}, \mu_{2}\right)}\right) v_{11}
$$

Demonstração: Aplicando-se a equação global de balanço ao conjunto de estados $(0,2) e(2,0)$ temos

$$
\left\{\begin{array}{l}
\left(\lambda+\mu_{2}\right) v_{02}=\mu_{1} v_{12}+\mu_{2} v_{03} \\
\left(\lambda+\mu_{1}\right) v_{20}=\mu_{1} v_{30}+\mu_{2} v_{21}
\end{array}\right.
$$

Substituindo-se os valores de $\mu_{1}, \mu_{2}$ do sistema de desigualdades (4.15) no sistema de equações acima temos:

$$
\left(\lambda+\mu_{2}\right) v_{02}=\mu_{1} v_{12}+\mu_{2} v_{03}
$$

portanto

$$
\left(\lambda+\min \left(\mu_{1}, \mu_{2}\right)\right) v_{02} \leq \max \left(\mu_{1}, \mu_{2}\right) v_{12}+\max \left(\mu_{1}, \mu_{2}\right) v_{03},
$$

ou seja,

$$
\left(\lambda+\min \left(\mu_{1}, \mu_{2}\right)\right) v_{02} \leq \max \left(\mu_{1}, \mu_{2}\right)\left(v_{12}+v_{03}\right)
$$

Da mesma forma, na segunda equação do sistema acima temos

$$
\left(\lambda+\mu_{1}\right) v_{20}=\mu_{1} v_{30}+\mu_{2} v_{21}
$$

portanto

$$
\left(\lambda+\min \left(\mu_{1}, \mu_{2}\right)\right)\left(v_{02}+v_{20}\right) \leq \max \left(\mu_{1}, \mu_{2}\right)\left(v_{30}+v_{21}\right)
$$


Somando-se as Equações (4.16) e (4.17) obtemos

$$
\begin{aligned}
& \left(v_{20}+v_{02}\right)\left(\lambda+\min \left(\mu_{1}, \mu_{2}\right)\right) \leq \max \left(\mu_{1}, \mu_{2}\right)\left(v_{03}+v_{12}+v_{21}+v_{30}\right), \\
& \left(v_{20}+v_{02}\right) \leq \frac{\max \left(\mu_{1}, \mu_{2}\right)}{\lambda+\min \left(\mu_{1}, \mu_{2}\right)}\left(v_{03}+v_{12}+v_{21}+v_{30}\right),
\end{aligned}
$$

aplicando-se o Lema 4.3.2 para $k=3$ temos

$$
\begin{aligned}
& \sum_{i+j=3} v_{i j} \leq\left(\frac{\lambda}{\min \left(\mu_{1}, \mu_{2}\right)}\right)\left(v_{02}+v_{11}+v_{20}\right) \\
&\left(v_{03}+v_{12}+v_{21}+v_{30}\right) \leq \frac{\lambda}{\min \left(\mu_{1}, \mu_{2}\right)}\left(v_{02}+v_{11}+v_{20}\right) \\
& v_{02}+v_{20} \leq\left(\frac{\max \left(\mu_{1}, \mu_{2}\right)}{\lambda+\min \left(\mu_{1}, \mu_{2}\right)}\right)\left(\frac{\lambda}{\min \left(\mu_{1}, \mu_{2}\right)}\right)\left(v_{02}+v_{11}+v_{20}\right) \\
& v_{02}+v_{20}-\left(\frac{\max \left(\mu_{1}, \mu_{2}\right)}{\lambda+\min \left(\mu_{1}, \mu_{2}\right)}\right)\left(\frac{\lambda}{\min \left(\mu_{1}, \mu_{2}\right)}\right)\left(v_{02}+v_{20}\right) \leq\left(\frac{\max \left(\mu_{1}, \mu_{2}\right)}{\lambda+\min \left(\mu_{1}, \mu_{2}\right)}\right)\left(\frac{\lambda}{\min \left(\mu_{1}, \mu_{2}\right)}\right) v_{11} \\
&\left(v_{02}+v_{20}\right)\left(1-\left(\frac{\max \left(\mu_{1}, \mu_{2}\right)}{\lambda+\min \left(\mu_{1}, \mu_{2}\right)}\right)\left(\frac{\lambda}{\min \left(\mu_{1}, \mu_{2}\right)}\right)\left(v_{02}+v_{20}\right)\right) \leq \\
&\left(\frac{\max \left(\mu_{1}, \mu_{2}\right)}{\lambda+\min \left(\mu_{1}, \mu_{2}\right)}\right)\left(\frac{\lambda}{\min \left(\mu_{1}, \mu_{2}\right)}\right) v_{11} .
\end{aligned}
$$

Seja $E_{i j}^{(e)}$ o tempo médio de permanência de um cliente esperto no estado $(i, j)$ quando usa a estratégia $e=1$ ou 3. Então

$$
E(T C S S \mid \text { estratégia } 1)=\sum_{i, j \in S} \nu_{i j} E_{i j}^{(1)}
$$

e

$$
E(T C S S \mid \text { estratégia } 3)=\sum_{i, j \in S} \nu_{i j} E_{i j}^{(3)}
$$

onde

$$
\begin{aligned}
& S=\{(i, j)|i \neq 0, j \neq 0,| i-j \mid \leq 1\} \\
& E^{(e)}=\sum_{(i, j) \in S} \nu_{i j} E_{i j}^{(e)}, \quad e=1,3 .
\end{aligned}
$$


Vamos demonstrar agora o Teorema 4.3.3.

Demonstração do Teorema (4.3.3): Fazendo-se a diferença entre o tempo médio de permanência do cliente esperto no sistema e utilizando-se a estratégia 3 e 1, temos

$$
\begin{aligned}
E(T C S S \mid \text { estratégia 3) }-E(T C S S \mid \text { estratégia 1) } & =\sum_{(i, j) \in S} \nu_{i j} E_{i j}^{(3)}-\sum_{(i, j) \in S} \nu_{i j} E_{i j}^{(1)} \\
& =E^{(3)}-E^{(1)}
\end{aligned}
$$

Definimos $P^{(e)}$ como sendo o tempo médio de permanência quando o cliente esperto chega no sistema e encontra um estado $(k, k)$ para todo $k \geq 2$, entra no sistema e utiliza uma das estratégias $e=1,3$. Assim temos

$$
\begin{gathered}
P^{(e)}=\sum_{(i, j) \in S} \nu_{i j} E_{i j}^{(e)}-v_{11} E_{11}^{(e)}=E^{(e)}-v_{11} E_{11}^{(e)}= \\
\sum_{k=2}^{\infty}\left(v_{k-1, k} E_{k-1, k}^{(e)}+v_{k, k-1} E_{k, k-1}^{(e)}\right)+\sum_{k=2}^{\infty} v_{k k} E_{k k}^{(e)} .
\end{gathered}
$$

Para a estratégia 3, suponhamos o caso em que o processo de chegada é Poisson. Sabemos da equação (4.12) que o número médio de clientes que chegam durante o intervalo de tempo em que $S$ observa é

$$
E(T C S S \mid \text { estratégia } 3)=\frac{1}{\mu_{1}+\mu_{2}}(E(N)+i+j+1) \quad \text { e } \quad E(N)=\frac{\lambda}{\mu_{1}+\mu_{2}}
$$

Vamos determinar o valor de $E_{k-1, k}^{(3)}$ que significa o tempo médio de permanência quando o cliente esperto chega no sistema e encontra na fila 1 e 2 com $k-1$ ou $k$ clientes. Então, temos

$$
\begin{aligned}
& E_{k \rightarrow 1, k}^{(3)}=\frac{1}{\mu_{1}+\mu_{2}}\left(\frac{\lambda}{\mu_{1}+\mu_{2}}+k-1+k+1\right)=\frac{\lambda}{\left(\mu_{1}+\mu_{2}\right)^{2}}+\frac{2 k}{\mu_{1}+\mu_{2}}, \\
& E_{k, k}^{(3)}=\frac{1}{\mu_{1}+\mu_{2}}\left(\frac{\lambda}{\mu_{1}+\mu_{2}}+k+k+1\right)=\frac{\lambda}{\left(\mu_{1}+\mu_{2}\right)^{2}}+\frac{2 k+1}{\mu_{1}+\mu_{2}} .
\end{aligned}
$$


Logo,

$$
E_{k-1, k}^{(3)}=E_{k, k-1}^{(3)}
$$

Substituindo-se esses resultados acima na Equação (4.18), temos

$$
P^{(3)}=\sum_{k=2}^{\infty}\left(\frac{\lambda}{\left(\mu_{1}+\mu_{2}\right)^{2}}+\frac{2 k}{\mu_{1}+\mu_{2}}\right)\left(v_{k-1, k}+v_{k, k-1}\right)+\sum_{k=2}^{\infty}\left(\frac{\lambda}{\left(\mu_{1}+\mu_{2}\right)^{2}}+\frac{2 k+1}{\mu_{1}+\mu_{2}}\right) v_{k k} .
$$

Aplicando-se o Lema 4.3.4 temos

$$
\begin{aligned}
P^{(3)}= & \sum_{k=2}^{\infty}\left(\frac{\lambda}{\left(\mu_{1}+\mu_{2}\right)^{2}}+\frac{2 k}{\mu_{1}+\mu_{2}}\right) \sum_{k=2}^{\infty}\left(v_{k \rightarrow 1, k}+v_{k, k-1}\right)+\sum_{k=2}^{\infty}\left(\frac{\lambda}{\left(\mu_{1}+\mu_{2}\right)^{2}}+\frac{2 k+1}{\mu_{1}+\mu_{2}}\right) v_{k k}, \\
P^{(3)} \leq & \sum_{k=2}^{\infty}\left(\frac{\lambda}{\left(\mu_{1}+\mu_{2}\right)^{2}}+\frac{2 k}{\mu_{1}+\mu_{2}}\right) \sum_{i+j=2 k \rightarrow 1} v_{i, j}+\sum_{k=2}^{\infty}\left(\frac{\lambda}{\left(\mu_{1}+\mu_{2}\right)^{2}}+\frac{2 k+1}{\mu_{1}+\mu_{2}}\right) \sum_{i+j=2 k} v_{i j} \leq \\
\leq & \left(\frac{\lambda}{\left(\mu_{1}+\mu_{2}\right)^{2}}+\frac{4}{\mu_{1}+\mu_{2}}\right) \sum_{i+j=3} v_{i, j}+\left(\frac{\lambda}{\left(\mu_{1}+\mu_{2}\right)^{2}}+\frac{6}{\mu_{1}+\mu_{2}}\right) \sum_{i+j=5} v_{i, j}+\ldots+ \\
& \left(\frac{\lambda}{\left(\mu_{1}+\mu_{2}\right)^{2}}+\frac{5}{\mu_{1}+\mu_{2}}\right) \sum_{i+j=4} v_{i, j}+\left(\frac{\lambda}{\left(\mu_{1}+\mu_{2}\right)^{2}}+\frac{7}{\mu_{1}+\mu_{2}}\right) \sum_{i+j=6} v_{i j}+\ldots,
\end{aligned}
$$

logo,

$$
P^{(3)} \leq \sum_{k=3}^{\infty}\left(\frac{\lambda}{\left(\mu_{1}+\mu_{2}\right)^{2}}+\frac{k+1}{\mu_{1}+\mu_{2}}\right) \sum_{i+j=k} v_{i, j}=
$$

aplicando-se o Lema 4.3 .5 temos

$$
P^{(3)} \leq \sum_{k=3}\left(\frac{\lambda}{\left(\mu_{1}+\mu_{2}\right)^{2}}+\frac{k+1}{\mu_{1}+\mu_{2}}\right)\left(\frac{\lambda}{\min \left(\mu_{1}, \mu_{2}\right)}\right)^{k-2} \sum_{i=0}^{2} v_{i, 2-i}
$$

Fazendo-se

$$
k_{1}=\sum_{k=3}^{\infty}\left(\frac{\lambda}{\left(\mu_{1}+\mu_{2}\right)^{2}}+\frac{k+1}{\mu_{1}+\mu_{2}}\right)\left(\frac{\lambda}{\min \left(\mu_{1}, \mu_{2}\right)}\right)^{k-2}
$$


temos

$$
P^{(3)} \leq k_{1}\left(v_{02}+v_{11}+v_{20}\right)
$$

Pelo Lema (4.3.5)

$$
\left(v_{02}+v_{20}\right)\left(1-\frac{\max \left(\mu_{1}, \mu_{2}\right)}{\lambda+\min \left(\mu_{1}, \mu_{2}\right)} \frac{\lambda}{\min \left(\mu_{1}, \mu_{2}\right)}\left(v_{02}+v_{20}\right)\right) \leq \frac{\max \left(\mu_{1}, \mu_{2}\right)}{\lambda+\min \left(\mu_{1}, \mu_{2}\right)} \frac{\lambda}{\min \left(\mu_{1}, \mu_{2}\right)} v_{11} .
$$

Fazendo-se

$$
M=\max \left(\mu_{1}, \mu_{2}\right), \quad m=\min \left(\mu_{1}, \mu_{2}\right), \quad L=\frac{\lambda}{m} \quad \text { e } \quad k_{2}=\frac{M}{\lambda+m} L
$$

obtemos

$$
\begin{gathered}
\left(v_{02}+v_{20}\right)\left(1-k_{2}\right) \leq k_{2} v_{11} \\
\left(v_{02}+v_{20}\right) \leq \frac{k_{2}}{1-k_{2}} v_{11}
\end{gathered}
$$

Somando-se $v_{11}$ em ambos os membros desta inequação temos

$$
v_{11}+v_{02}+v_{20} \leq \frac{1}{1-k_{2}} v_{11}
$$

Para a estratégia 1,

$$
E(T C S S \mid \text { estratégia } 1)=\frac{1}{2 \mu_{1}}+\frac{1}{2 \mu_{2}} .
$$

Vamos determinar o valor de $E_{k-1, k}^{(1)}, E_{k, k-1}^{(1)}$ e $E_{k, k}^{(1)}$ que significa o tempo médio de permanência que o cliente esperto chega no sistema e encontra nas filas $1 e 2, k-1$ ou $k$ clientes. Então, temos

$$
E_{k-1, k}^{(1)}=\frac{k}{\mu_{1}}, \quad E_{k, k-1}^{(1)}=\frac{k}{\mu_{2}}, \quad \text { e } \quad E_{k, k}^{(1)}=\frac{k+1}{\mu_{1}}+\frac{k+1}{\mu_{2}} .
$$


Substituindo esses resultados na equação (4.18) temos

$$
\begin{aligned}
P^{(1)} & =\sum_{k=2}^{\infty}\left(v_{k-1, k} E_{k-1, k}^{(1)}+v_{k, k-1} E_{k, k-1}^{1}\right)+\sum_{k=2}^{\infty} v_{k k} E_{k k}^{(1)} \leq \\
& \leq \sum_{k=2}^{\infty}\left(\frac{k}{\mu_{1}}+\frac{k}{\mu_{2}}\right) \sum_{i+j=2 k-1} v_{i j}+\sum_{k=2}^{\infty}\left(\frac{k+1}{2 \mu_{1}}+\frac{k+1}{2 \mu_{2}}\right) \sum_{i+j=2 k} v_{i j} \leq \\
& \leq \sum_{k=2}^{\infty}\left(\frac{1}{\mu_{1}}+\frac{1}{\mu_{2}}\right)\left(\frac{2 k-1}{2}+1\right) \sum_{i+j=2 k-1} v_{i j}+\sum_{k=2}^{\infty}\left(\frac{1}{\mu_{1}}+\frac{1}{\mu_{2}}\right)\left(\frac{2 k}{2}+1\right) \sum_{i+j=2 k} v_{i j} .
\end{aligned}
$$

Logo,

$$
\begin{aligned}
& P^{(1)} \leq\left(\frac{1}{\mu_{1}}+\frac{1}{\mu_{2}}\right) \sum_{k=2}^{\infty}\left(\frac{k}{2}+1\right) \sum_{i+j=2 k} v_{i j} . \\
& P^{(1)} \leq\left(\frac{1}{\mu_{1}}+\frac{1}{\mu_{2}}\right) \sum_{k=3}^{\infty}\left(\frac{k}{2}+1\right) \sum_{i+j=k} v_{i j},
\end{aligned}
$$

aplicando-se o Lema 4.3.4 temos

$$
P^{(1)} \leq\left(\frac{1}{\mu_{1}}+\frac{1}{\mu_{2}}\right) \sum_{k=3}^{\infty}\left(\frac{k}{2}+1\right) L^{k-2} \sum_{i+j=2} v_{i j} .
$$

Desenvolvendo-se o primeiro somatório do segundo membro dessa desigualdade temos

$$
\begin{aligned}
\sum_{k=3}^{\infty}\left(\frac{k}{2}+1\right) L^{k-2} & =\frac{1}{2} \sum_{k=3}^{\infty} k L^{k-2}+\sum_{k=3}^{\infty} L^{k-2}= \\
& =\frac{1}{2}\left(\sum_{k=3}^{\infty}(k-2+2) L^{k-2}\right)+L^{k-2}= \\
& =\frac{1}{2} \sum_{k=3}^{\infty}(k-2) L^{k-2}+2 \sum_{k=3}^{\infty} L^{k-2} .
\end{aligned}
$$

Logo,

$$
\sum_{k=3}^{\infty}\left(\frac{k}{2}+1\right) L^{k-2}=\frac{1}{2} \sum_{k=1}^{\infty}(k) L^{k}+2 \sum_{k=1}^{\infty} L^{k}
$$


Derivando-se e multiplicando-se por $\mathrm{x}, \quad|x|<1$, ambos os membros da equaçāo $\frac{1}{1-x}=\sum_{n=0}^{\infty} x^{n}$ e substituindo-se em (4.20) obtemos

$$
\begin{aligned}
\sum_{k=3}^{\infty}\left(\frac{k}{2}+1\right) L^{k-2} & =\frac{L}{(1-L)^{2}}+2 \frac{L}{1-L}= \\
& =L\left[\frac{1}{2}(1-L)^{-2}+2(1-L)^{-1}\right]
\end{aligned}
$$

substituindo-se esse resultado na equação (4.20) temos

$$
P^{(1)} \leq\left(\frac{1}{\mu_{1}}+\frac{1}{\mu_{2}}\right) L\left[\frac{1}{2}(1-L)^{-2}+2(1-L)^{-1}\right]\left(v_{02}+v_{20}+v_{11}\right) .
$$

Fazendo-se

$$
k_{3}=\left(\frac{1}{\mu_{1}}+\frac{1}{\mu_{2}}\right) L\left[\frac{1}{2}(1-L)^{-2}+2(1-L)^{-1}\right]
$$

e usando (4.19), temos

$$
P^{(1)} \leq k_{3}\left(\frac{1}{1-k_{2}}\right) v_{11}
$$

Vamos determinar a diferença entre o $E^{(3)}$ e $E^{(1)}$ :

$$
E^{(3)}-E^{(1)}=P^{(3)}+v_{11} E_{11}^{(3)}-P^{(1)}-v_{11} E_{11}^{(1)}=v_{11}\left(E_{11}^{(3)}-E_{11}^{(1)}\right)+P^{(3)}-P^{(1)}(4
$$

Calculando-se os valores de $E_{11}^{(3)}$ e $E_{11}^{(1)}$ temos

$$
\begin{aligned}
& E_{11}^{(3)}=\frac{1}{\mu_{1}+\mu_{2}}\left(\frac{\lambda}{\mu_{1}+\mu_{2}}+3\right)=\left(\frac{\lambda}{\left(\mu_{1}+\mu_{2}\right)^{2}}+\frac{3}{\mu_{1}+\mu_{2}}\right) \\
& E_{11}^{(1)}=\frac{2}{2 \mu_{1}}+\frac{2}{2 \mu_{2}}=\frac{1}{\mu_{1}}+\frac{1}{\mu_{2}}
\end{aligned}
$$

Substituindo-se esses resultados na equação (4.22) temos

$$
E^{(3)}-E^{(1)}=v_{11}\left(\frac{\lambda}{\left(\mu_{1}+\mu_{2}\right)^{2}}+\frac{3}{\mu_{1}+\mu_{2}}-\frac{1}{\mu_{1}}-\frac{1}{\mu_{2}}\right)+\left(P^{(3)}-P^{(1)}\right) .
$$


De acordo com Lema 4.3.3.

Como a desigualdade

$$
\left|P^{(3)}-P^{(1)}\right| \leq P^{(3)}+P^{(1)}, \quad \text { pois } \quad P^{(3)} \geq 0, \quad P^{(1)} \geq 0
$$

temos

$$
P^{(3)}-P^{(1)} \leq\left(\frac{k_{1}+k_{3}}{1-k_{2}}\right) v_{11}
$$

Analisando-se a diferença entre $E_{11}^{(3)}$ e $E_{11}^{(1)}$ :

$$
\frac{3}{\mu_{1}+\mu_{2}}-\frac{1}{\mu_{1}}-\frac{1}{\mu_{2}}=\frac{-\mu_{1} \mu_{2}-\left(\mu_{1}-\mu_{2}\right)^{2}}{\left(\mu_{1}+\mu_{2}\right) \mu_{1} \mu_{2}}<0,
$$

concluímos que essa diferença é sempre negativa.

Definindo-se

$$
k_{4}\left(\mu_{1}, \mu_{2}\right)=\frac{3}{\mu_{1}+\mu_{2}}-\frac{1}{\mu_{1}}-\frac{1}{\mu_{2}} .
$$

Concluímos imediatamente pelo Teorema 4.3.3 os limites abaixo:

$$
\begin{aligned}
& \lim _{\mu_{1} \rightarrow \infty} \frac{\frac{\lambda}{\left(\mu_{1}+\mu_{2}\right)^{2}}}{\left|k_{4}\left(\mu_{1}, \mu_{2}\right)\right|}=0, \\
& \lim _{\mu_{1} \rightarrow \infty} \frac{k_{1}\left(\lambda, \mu_{1}, \mu_{2}\right)}{\mid k_{4}\left(\mu_{1}, \mu_{2}\right)}=0, \\
& \lim _{\mu_{1} \rightarrow \infty} \frac{k_{3}\left(\lambda, \mu_{1}, \mu_{2}\right)}{\left|k_{4}\left(\mu_{1}, \mu_{2}\right)\right|}=0
\end{aligned}
$$


e

$$
\lim _{\mu_{1} \rightarrow \infty} \frac{1}{1-k_{2}}=1
$$

A partir desses resultados concluímos que o termo

$$
\left(\frac{3}{\mu_{1}+\mu_{2}}-\frac{1}{\mu_{1}}-\frac{1}{\mu_{2}}\right) v_{11}
$$

da equação (4.22) domina o outro termo da mesma equação quando $\mu_{1} \rightarrow \infty$. A partir da equação (4.24) concluímos que a diferença entre o tempo médio de permanência que o cliente que entra no sistema utilizando a estratégia 3 ou 1 , isto é, $E^{(3)}-E^{(1)}$ é sempre negativo, para a taxa $\mu_{1}$ suficientemente grande.

\subsection{Resultados Numéricos}

Nesta seçāo apresentamos os resultados e a análise do tempo médio de permanência do cliente esperto para o sistema de fila $M /((M+M) / 1)^{2}$. Fixemos para o sistema o tempo médio de serviço do primeiro servidor em 1 e assumimos os níveis de ocupação $\rho=\frac{\lambda}{\mu_{1}+\mu_{2}}$ variando de 0,1 a 0,9 . Vamos determinar os conjuntos dos pares $\mu_{2}$ e $\lambda$ que satisfazem o Teorema 4.1.1. De acordo com este teorema, se uma das taxas for pequena e outra grande, a estratégia 2 apresenta um tempo médio de permanência do cliente esperto melhor do que na estratégia 1.

Na Tabela 4.1 apresentamos os resultados de $\mu_{2}$ e $\lambda$ em negrito, o que satisfaz o teorema 4.1, considerando-se o limite quando o tempo médio de permanência do cliente esperto é igual em ambas as estratégias (1 e 2). A Figura 4.5 mostra as raízes da equação (4.26) para $\rho=0,1$.

$$
\frac{1}{2}+\frac{1}{2 \mu_{2}}=\frac{10}{1+\mu_{2}}+1,155\left(\frac{1}{1+0,1\left(1+\mu_{2}\right)}+\frac{1}{\mu_{2}+0,1\left(1-\mu_{2}\right)}\right)
$$

Na Tabela 4.2 apresentamos os resultados comparativos dos tempos médios de permanência do cliente esperto sob as estratégias 1 e 2. Para o sistema $M /((M+M) / 1)^{2}$ 


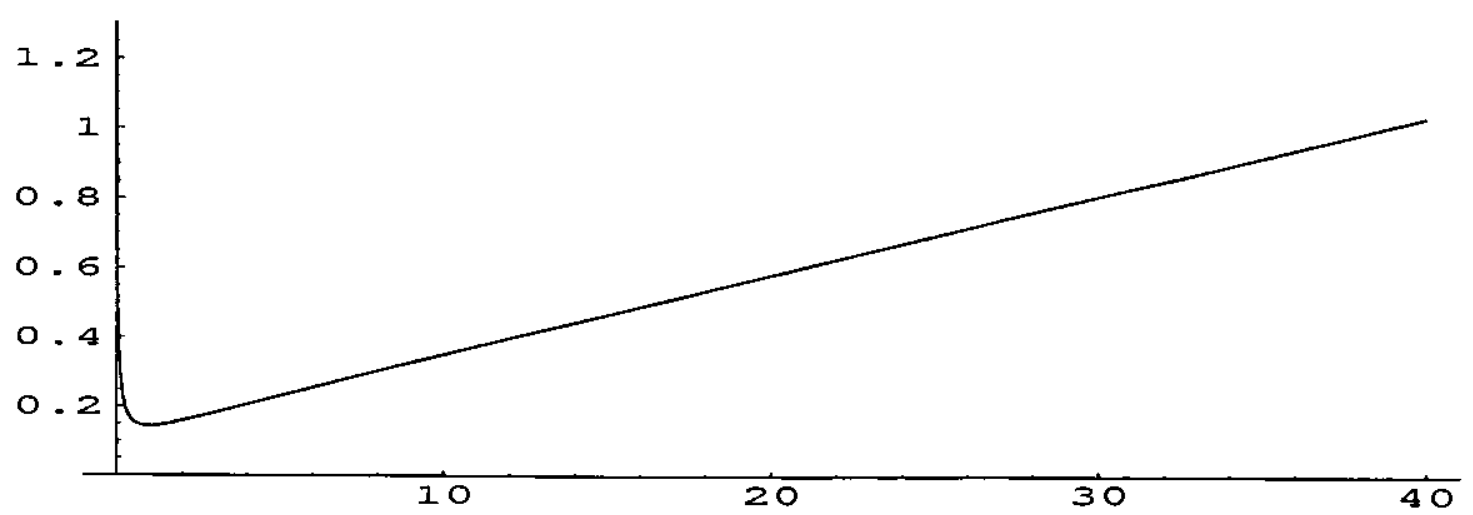

Figura 4.5: Valores de $\mu_{2}$ que satisfazem o teorema 4.1

verificamos que para valores fora das raízes o tempo médio de permanência do cliente esperto sob a estratégia 1 é maior. Para os valores de $\rho>0,1, \mu_{2}=0,020$ e 0,026 , $\rho>0,6, \mu_{2}=0,068, \rho>0,8$ e $\mu_{2}=0,080$ temos que o tempo médio de permanência do cliente esperto sob a estratégia de observar as filas paralelas antes de unir-se a uma delas é melhor do que a estratégia de unir-se à fila mais curta.

Concluímos que para os valores entre as raízes que satisfazem o Teorema 4.1.1, o tempo médio de permanência do cliente esperto sob a estratégia 1 é melhor. Para $\mu_{2} \rightarrow \infty$ temos que o tempo médio que o cliente permanece no sistema sob a estratégia 2 é sempre menor do que na estratégia 1.

Tabela 4.1 - Os valores de $\mu_{2}$ e $\lambda$

\begin{tabular}{|c|c|c|c|c|c|}
\hline$\rho$ & $\mu_{1}$ & $\mu_{2}^{\prime}$ & $\mu_{2}^{\prime \prime}$ & $\lambda^{\prime}$ & $\lambda^{\prime \prime}$ \\
\hline 0,1 & 1 & $\mathbf{0 , 0 2 5 9 3}$ & 38,563 & $\mathbf{0 , 1 0 3}$ & 3,956 \\
0,2 & 1 & $\mathbf{0 , 0 4 7 6 3}$ & 20,994 & $\mathbf{0 , 2 1 0}$ & 4,399 \\
0,3 & 1 & $\mathbf{0 , 0 6 5 4 1}$ & 15,289 & $\mathbf{0 , 3 2 0}$ & 4,887 \\
04 & 1 & $\mathbf{0 , 0 7 9 7 1}$ & 12,545 & $\mathbf{0 , 4 3 2}$ & 5,418 \\
0,5 & 1 & $\mathbf{0 , 0 9 1 0 4}$ & 10,983 & $\mathbf{0 , 5 4 6}$ & 5,991 \\
0,6 & 1 & $\mathbf{0 , 0 9 9 8 8}$ & 10,012 & $\mathbf{0 , 6 6 0}$ & 6,607 \\
0.7 & 1 & $\mathbf{0 , 1 0 6 6 5}$ & 9,376 & $\mathbf{0 , 7 7 5}$ & 7,263 \\
0,8 & 1 & $\mathbf{0 , 1 1 1 7 2}$ & 8,951 & $\mathbf{0 , 8 8 9}$ & 7,961 \\
0,9 & 1 & 0,1154 & 8,665 & 1,004 & 8,698 \\
\hline
\end{tabular}


Tabela 4.2 - O Tempo Médio de espera do cliente esperto na estratégia 1 e 2

\begin{tabular}{|c|c|c|c|c|c|}
\hline$\rho$ & $E(T C C S \mid 2)$ & $E(T C C S \mid 2)$ & $E(T C C S \mid 2)$ & $E(T C C S \mid 2)$ & $E(T C C S \mid 2)$ \\
\hline 0.1 & 32,45 & 32,26 & 31,71 & 30,99 & 30,65 \\
0,2 & 17,84 & 17,74 & 17,43 & 17,04 & 16,85 \\
0,3 & 13,04 & 12,96 & 12,74 & 12,45 & 13,31 \\
0,4 & 10,69 & 10,62 & 10,44 & 10,21 & 10,09 \\
0,5 & 9,31 & 9,26 & 9,10 & 8,90 & 8,80 \\
0,6 & 8,34 & 8,38 & 8,23 & 8,05 & 7,96 \\
0,7 & 7,83 & 7,78 & 7,64 & 7,48 & 7,39 \\
0,8 & 7,40 & 7,36 & 7,23 & 7,07 & 6,99 \\
0.9 & 4,10 & 7,05 & 6,93 & 6,77 & 6,69 \\
\hline$E(T C C S \mid 1)$ & 25,5 & 19,73 & 11,86 & 7,85 & 6,75 \\
\hline$\mu_{2}$ & 0,020 & 0,026 & 0,044 & 0,068 & 0,080 \\
\hline
\end{tabular}

\subsection{Considerações Finais}

Neste capítulo analisamos o tempo médio de permanência do cliente esperto para o sistema $G /(M+M) / 1)^{2}$. No próximo capítulo apresentamos uma nova proposta de observar filas paralelas de serviços gerais antes de unir-se a elas. 


\section{Capítulo 5}

\section{Observando Filas Paralelas com Serviço Geral}

\subsection{Introdução}

Neste capítulo apresentamos um sistema de fila $M /((M+G) / 1)^{2}$, com um cliente esperto usando uma estratégia de esperar e observar duas filas paralelas antes de unirse a uma delas, e os demais clientes utilizando a estratégia de unir-se à fila mais curta. Analisamos o tempo médio de permanência do cliente esperto no sistema, para as duas estratégias distintas. Neste sentido, apresentamos na Seção 5.2 o modelo e a notação de filas paralelas que estudamos no artigo de Stanford e Hlynka(96), Observing General Service Queues before Joining, cujo propósito é mostrar que o tempo médio de permanência do cliente esperto no sistema sob a estratégia de observar as filas antes de unir-se a elas é menor do que a estratégia de unir-se à fila mais curta. Neste capítulo analisamos o tempo médio de permanência do cliente esperto no sistema sem considerar o limite do tempo de serviço, enquanto no capítulo anterior essa consideraçao do limite foi feita. Na Seção 5.3 e 5.4 demonstramos como calcular o tempo médio de permanência do cliente esperto no sistema. Na seção 5.5 são apresentadas as tabelas comparativas do tempo médio de permanência do cliente esperto no sistema e na seção 5.6, as considerações finais. 


\subsection{Modelo}

Nesta seção descrevemos inicialmente o sistema de fila $M /((M+G) / 1)^{2}$, que apresenta as seguintes características: 2 servidores cada um com sua própria fila; o primeiro servidor atende aos clientes com tempo de serviço distribuído exponencialmente e o segundo com uma distribuiçāo geral i.i.d; as salas de espera são ilimitadas; os tempos entre as chegadas são variáveis aleatórias exponenciais i.i.d. Os tempos entre as chegadas e os tempos de serviço de cada um dos servidores são independentes.

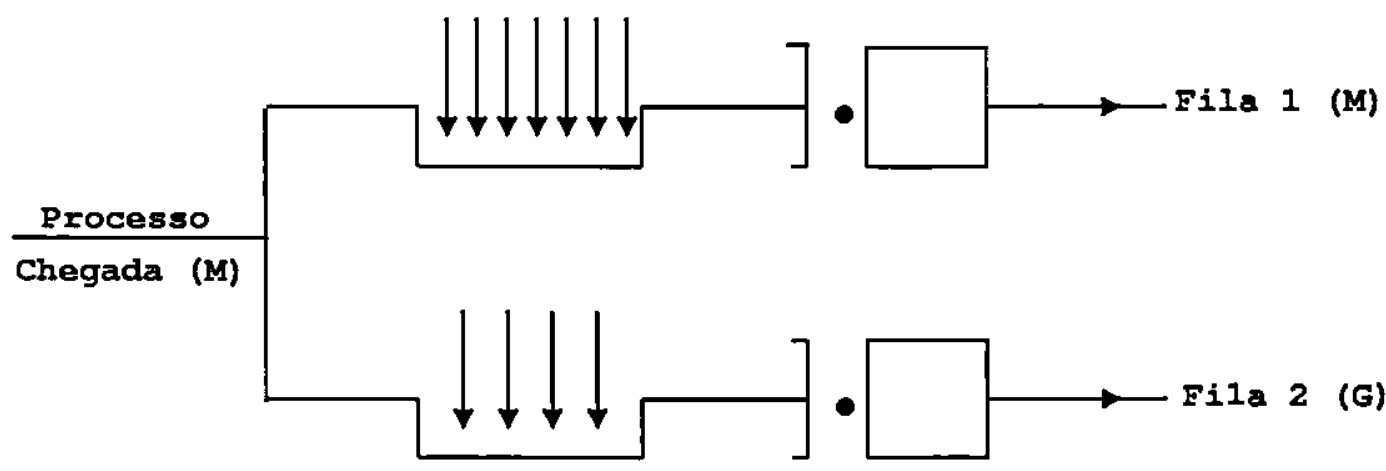

Figura 5.1: Uma representaçāo do modelo $M /((M+G) / 1)^{2}$

Este capítulo avalia o tempo médio que um cliente esperto permanece no sistema de fila $M /((M+G) / 1)^{2}$ em equilíbrio. Para isso, devemos analisar uma única estratégia de como o cliente esperto deve entrar no sistema.

\subsection{Análise do Modelo com um Servidor com Tempo de Serviço Exponencial}

Primeiramente vamos calcular o tempo médio de permanência do cliente esperto no sistema, considerando o sistema vazio. Os clientes que chegam após o cliente esperto no 
sistema escolhem a estratégia de unir-se à fila mais curta, exceto para o cliente esperto que toma uma decisão conforme a estratégia a seguir. Seja $S$ o cliente esperto.

Estratégia de S. Consideremos $n_{l}$ o número de clientes na fila $l, l=1,2$. Se ambas as filas forem não vazias e $\left|n_{1}-n_{2}\right|>1$, então $S$ se junta à fila mais curta. Se ambas as filas forem não vazias e $\left|n_{1}-n_{2}\right| \leq 1$, então $S$ espera pela conclusão do primeiro serviço e juntase à fila na qual a conclusão aconteceu. Se exatamente uma das filas estiver vazia, então da mesma forma ele espera por uma conclusão de serviço. Se ambas as filas estiverem vazias, $S$ espera até um outro cliente ou outros clientes chegarem e entrarem no sistema.

Enunciaremos alguns lemas e teoremas para calcular o tempo médio de permanência do cliente esperto no sistema para essa estratégia. Para isto, consideremos a seguinte notação:

$S$ : o cliente esperto;

$E(T C S)$ : o tempo médio de permanência do cliente esperto no sistema;

$E(T)$ : o tempo médio entre as chegadas;

$C_{1}:$ o primeiro cliente a chegar após $S$;

$C_{2}$ : o segundo cliente a chegar após $S$;

$i$ : a fila na qual $C_{\mathrm{I}}$ escolhe e começa a ser atendido, assumindo valores $i=1,2$;

$E(Q \mid i)$ : o tempo médio entre o início do serviço de $C_{1}$, no servidor $i$, e o final do serviço de $S$.

Teorema 5.3.1. Sejam $E(T C S), E(T)$ e $E(Q \mid i)$ definidos acima para $i=1$, 2. Então,

$$
E(T C S)=E(T)+\frac{1}{2}(E(Q \mid 1)+E(Q \mid 2)) .
$$

Demonstração: Vamos inicialmente calcular o valor de $E(Q \mid 1)$ desta equação. Consideremos que o cliente esperto chega, encontra o sistema vazio e espera antes de juntar-se a uma das filas. Temos dois casos possíveis: no primeiro caso $C_{1}$, está sendo atendido pelo servidor da fila 1 e termina o serviço antes de $C_{2}$. Logo, o cliente esperto espera a conclusão do serviço de $C_{1}$ e seleciona a fila 1, conforme Figura 5.2. 
No segundo caso ambos os servidores estão ocupados, os novos clientes chegam ao sistema e escolhem uma das filas, conforme o seguinte critério: o cliente junta-se à fila mais curta; se as filas tiverem o mesmo comprimento, a escolha é aleatória. Considerando que a partir do momento em que ambos servidores estejam ocupados, $S$ terá um tempo de observação mais um tempo de espera na fila e um tempo de serviço, devido aos clientes que entraram na frente do mesmo. Assim, o número médio dos clientes que ficam à frente de $S$ em cada uma das filas é $\lambda x / 2$, onde $x$ é o tempo de observação a partir do momento em que ambos servidores estejam ocupados até a conclusão do primeiro serviço, conforme a Figura 5.3.

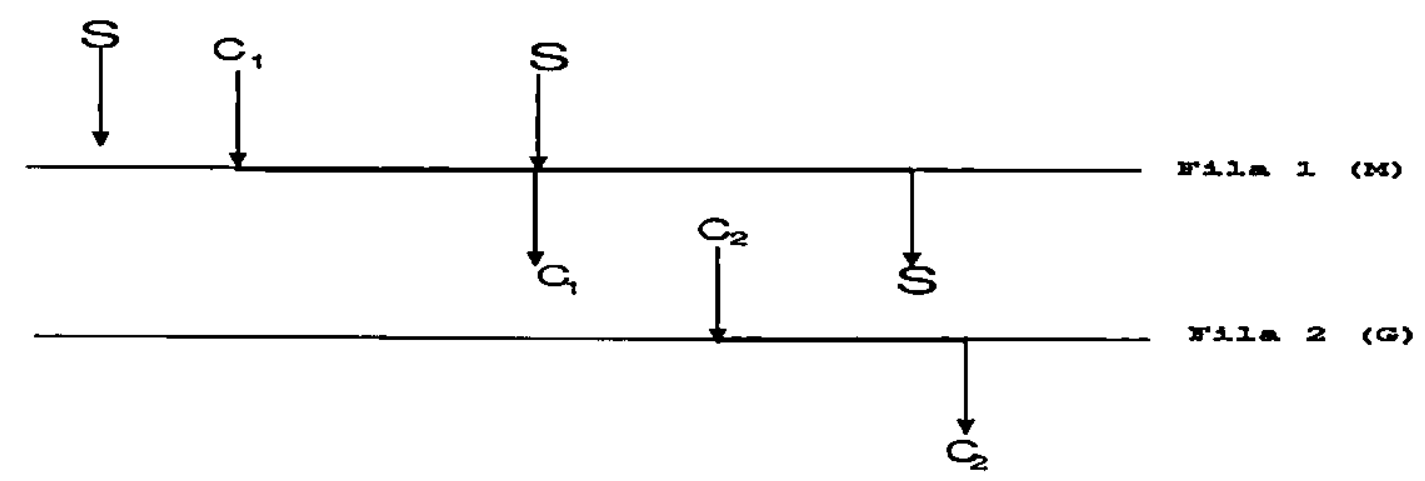

Figura 5.2: Uma realização simples do modelo

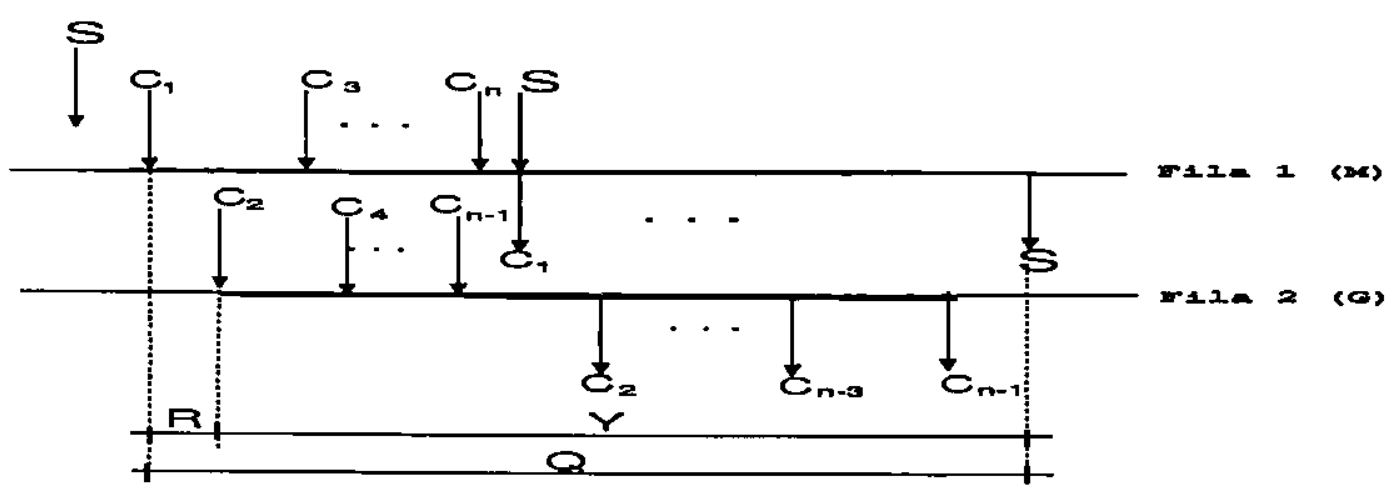

Figura 5.3: Representação de uma realização com serviço nos dois servidores

Seja então:

$Y$ : a variável aleatória que representa o tempo de espera no sistema a partir do instante em que ambos servidores estejam ocupados até $S$ completar o serviço; 
$E(Y \mid 1)$ : o tempo médio desde do inicio do serviço de $C_{2}$, até que $S$ complete o serviço;

$X_{2}$ : o tempo de serviço geral do servidor 2 ;

$\phi_{X_{2}}(s)$ : a transformada de Laplace-Stieltjes da distribuiçāo do tempo de serviço para o servidor geral.

Lema 5.3.1. Sejam $E(Y \mid 1)$ e $E(Q \mid 1)$ definidos acima, então

(a) $E(Y \mid 1)=\left(1-\phi_{X_{2}}\left(\mu_{1}\right)\right) A+E\left(X_{2}\right) \phi_{X_{2}}\left(\mu_{1}\right)-\left.B \frac{d}{d s} \phi_{X_{2}}(s)\right|_{\left(s=\mu_{1}\right)}$,

onde

$$
\begin{aligned}
\left.A=\left(\frac{1}{\mu_{1}}\right)\left(2+\frac{\lambda}{2 \mu_{1}}\right) \text { e } B=\left(\frac{\lambda}{2}\right)\left(E\left(X_{2}\right)-\frac{1}{\mu_{1}}\right)\right) ; \\
\text { (b) } E(Q \mid 1)=\frac{2}{\mu_{1}+\lambda}+\frac{\lambda}{\mu_{1}+\lambda}(E(Y \mid 1)) .
\end{aligned}
$$

Demonstração: Suponhamos que o tempo residual de $C_{1}$ seja menor e maior ou igual ao tempo de serviço do servidor 2 , conforme as figuras (5.4) e (5.5).

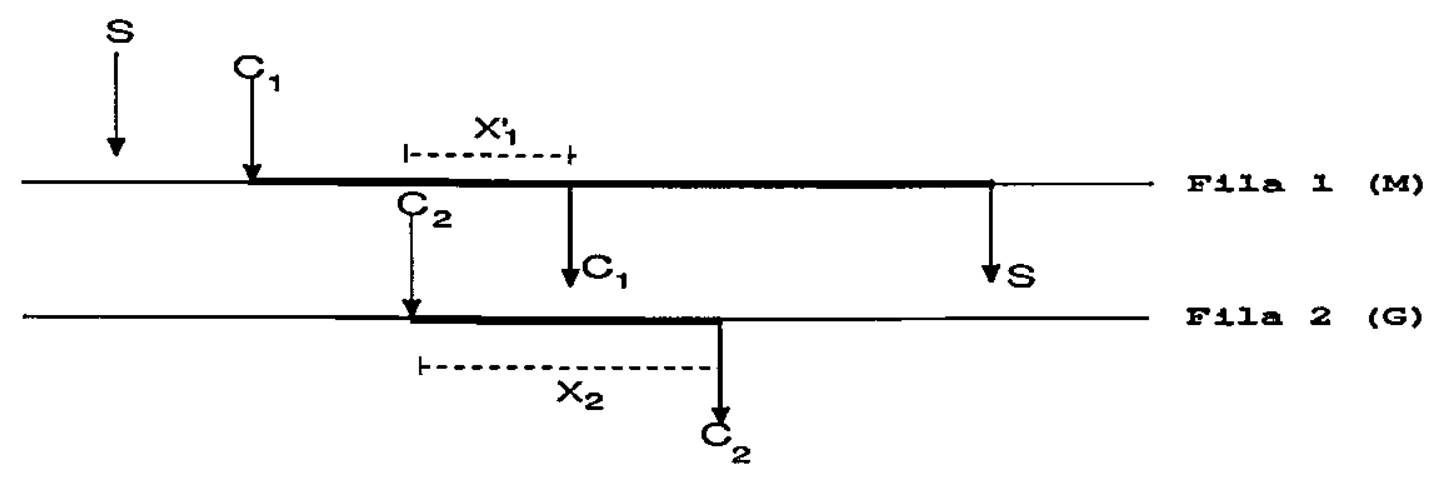

Figura 5.4: Uma realização com tempo residual do serviço de $C_{1}$ menor do que o serviço de $C_{2}$

Analisando os dois casos possíveis quando o cliente $C_{1}$ escolhe a fila 1 , o valor de $E(Y \mid 1)$ é dado por

$$
E(Y \mid 1)=E\left(Y * I\left(X_{1}^{\prime}<X_{2}\right) \mid 1\right)+E\left(Y * I\left(X_{1}^{\prime} \geq X_{2}\right) \mid 1\right),
$$

onde: 
- $X_{1}^{\prime}$ : é o tempo de serviço residual na fila 1 a partir do momento em que ambos servidores estejam ocupados;

- $Y * I\left(X_{1}^{\prime}<X_{2}\right)$ : indicando que $S$ seleciona a fila 1 , pois o cliente que chegou após $C_{1}$ terminou o serviço depois deste (Figura 5.4 );

- $Y * I\left(X_{1}^{\prime} \geq X_{2}\right)$ : indicando que $S$ seleciona a fila 2 , pois o cliente que chegou após $C_{1}$ terminou o serviço antes deste (Figura 5.5 ).

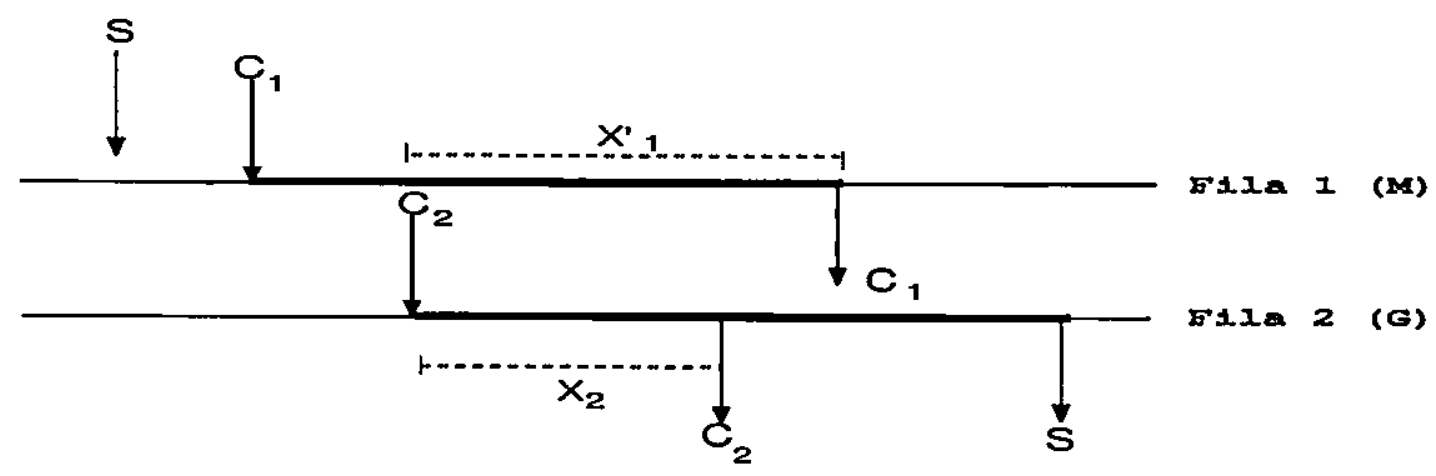

Figura 5.5: Uma realização com tempo residual do serviço de $C_{1}$ maior do que o serviço de $C_{2}$

O primeiro termo do segundo membro de (5.2) é

$$
\begin{aligned}
& E\left(Y * I\left(X_{1}^{\prime}<X_{2}\right) \mid 1\right)=\int_{0}^{\infty} \int_{0}^{t}\left(\tau+\left(\frac{\lambda \tau}{2}+1\right)\left(1 / \mu_{1}\right)\right) \mu_{1} e^{-\mu_{1} \tau} d \tau d P\left[X_{2} \leq t\right]= \\
& =\left(1+\frac{\lambda}{2 \mu_{1}}\right) \int_{0}^{\infty} \int_{0}^{t} \tau \mu_{1} e^{-\mu_{1} \tau} d \tau d P\left[X_{2} \leq t\right]+\frac{1}{\mu_{1}} \int_{0}^{\infty} \int_{0}^{t} \tau \mu_{1} e^{-\mu_{1} \tau} d \tau d P\left[X_{2} \leq t\right]= \\
& =\left(1+\frac{\lambda}{2 \mu_{1}}\right) \int_{0}^{\infty}\left(-\int_{0}^{t} \tau d e^{-\mu_{1} \tau}\right) d P\left[X_{2} \leq t\right]+\frac{1}{\mu_{1}} \int_{0}^{\infty} \int_{0}^{t} \mu_{1} e^{-\mu_{1} \tau} d \tau d P\left[X_{2} \leq t\right]= \\
& =\left(1+\frac{\lambda}{2 \mu_{1}}\right) \int_{0}^{\infty} \frac{1}{\mu_{1}}\left(1-\left(1+\mu_{1} t\right) e^{-\mu_{1} t}\right) d P\left[X_{2} \leq t\right]+\frac{1}{\mu_{1}} \int_{0}^{\infty}\left(1-e^{-\mu_{1} t}\right) d P\left[X_{2} \leq t\right] .
\end{aligned}
$$

Logo,

$$
E\left(Y * I\left(X_{1}^{\prime}<X_{2}\right) \mid 1\right)=\frac{1}{\mu_{1}}\left(1-\phi_{X_{2}}\left(\mu_{1}\right)\right)+\left\{\left(1+\frac{\lambda}{2 \mu_{1}}\right) \frac{1}{\mu_{1}}\right\}\left(1-\phi_{X_{2}}\left(\mu_{1}\right)\right)+\left.\mu_{1} \frac{d \phi_{X_{2}}(s)}{d s}\right|_{s=\mu_{1}} .
$$

Por outro lado, o segundo termo é

$$
E\left(Y * I\left(X_{1}^{\prime} \geq X_{2}\right) \mid 1\right)=\int_{0}^{\infty} e^{-\mu_{1} t}\left(t+\left(\frac{\lambda t}{2}+1\right) E\left(X_{2}\right)\right) d P\left[X_{2} \leq t\right]=
$$




$$
\begin{aligned}
& =\left(1+\frac{\lambda E\left(X_{2}\right)}{2}\right) \int_{0}^{\infty} t e^{-\mu_{1} t} d P\left[X_{2} \leq t\right]+E\left(X_{2}\right) \int_{0}^{\infty} t e^{-\mu_{1} t} P\left[X_{2} \leq t\right]= \\
& \left.\left.\left.=\left.\left(1+\frac{\lambda E\left(X_{2}\right)}{2}\right)\left(-\frac{d}{d s} \phi_{X_{2}(s)}\right)\right|_{s=\mu_{1}}\right)+E\left(X_{2}\right)\right) \phi_{X_{2}}\left(\mu_{1}\right)\right) .
\end{aligned}
$$

Assim, somando-se os dois termos, obtemos

$$
E(Y \mid 1)=\left(2+\frac{\lambda}{2 \mu_{1}}\right)\left(\frac{1}{\mu_{1}}\right)\left(1-\phi_{X_{2}}\left(\mu_{1}\right)\right)-\left.\frac{\lambda}{2}\left(E\left(X_{2}\right)-\frac{1}{\mu_{1}}\right) \frac{d}{d s} \phi_{X_{2}}(s)\right|_{s=\mu_{1}}
$$

Sejam

$$
A=\left(2+\frac{\lambda}{2 \mu_{1}}\right)\left(\frac{1}{\mu_{1}}\right) \quad \text { e } \quad B=\frac{\lambda}{2}\left(E\left(X_{2}\right)-\frac{1}{\mu_{1}}\right) .
$$

Substituindo-se na equação acima temos

$$
E(Y \mid 1)=\left(1-\phi_{X_{2}}\left(\mu_{1}\right)\right) A-\left.B \frac{d}{d s} \phi_{X_{2}}(s)\right|_{s=\mu_{1}}
$$

O que termina a prova da primeira parte do lema.

Vamos agora à segunda parte do lema. Para isso, precisamos calcular o tempo médio de permanência do $C_{1}$ na fila 1 , até a próxima ocorrência (chegada ou conclusão de serviço), à qual designaremos por $E(R \mid 1)$. Temos

$$
\begin{aligned}
E(R \mid 1) & =\int_{0}^{\infty} P(R>t \mid 1) d t=\int_{0}^{\infty} P\left[\min \left\{\text { tempo } C_{2}, \text { serviço } C_{1}\right\}\right] d t= \\
& =\int_{0}^{\infty} P\left(\text { tempo até a chegada de } C_{2}>t \mid 1\right) P\left(\text { serviço } C_{1}\right) d t= \\
& =\int_{0}^{\infty} e^{-\lambda t} e^{-\mu_{1} t} d t=\int_{0}^{\infty} e^{-\left(\lambda+\mu_{1}\right) t} d t=\frac{1}{\lambda+\mu_{1}} .
\end{aligned}
$$

A probabilidade para que a próxima ocorrência seja uma conclusão de serviço, sendo que o primeiro cliente escolheu o servidor 1, a qual denotaremos por $P\left(A_{1} \mid 1\right)$ é

$$
\begin{aligned}
P\left(A_{1} \mid 1\right) & =\int_{0}^{\infty} P\left(\text { tempo até a chegada } C_{2}>t\right) P\left(\text { serviço } C_{1}<t\right) d t= \\
& =\int_{0}^{\infty} e^{-\lambda t} \mu_{1} e^{-\mu_{1} t} d t=\frac{\mu_{1}}{\lambda+\mu_{1}} .
\end{aligned}
$$


Portanto, o tempo médio medido a partir do início do serviço de $C_{1}$ na fila 1 até $S$ completar o serviço é obtido da seguinte forma:

$$
\begin{aligned}
E(Q \mid 1) & =E(R \mid 1)+P\left(A_{1} \mid 1\right) \frac{1}{\mu_{1}}+\left(1-P\left(A_{1} \mid 1\right)\right) E(Y \mid 1)= \\
& =\frac{1}{\lambda+\mu_{1}}+\frac{1}{\lambda+\mu_{1}}+\left(1-\frac{\mu_{1}}{\lambda+\mu_{1}}\right) E(Y \mid 1)= \\
& =\frac{2}{\lambda+\mu_{1}}+\left(\frac{\lambda}{\lambda+\mu_{1}}\right) E(Y \mid 1) .
\end{aligned}
$$

Completa-se a prova do lema.

Corolário 5.3.1. Em particular, para o sistema de fila $M /(M / 1)^{2}$, onde $C_{1}$ seleciona a fila 1, o tempo médio desde o instante em que ambos os servidores estão ocupados até que $S$ complete o serviço é dado por:

$$
E(Y \mid 1)=\frac{3}{\mu_{2}+\mu_{1}}+\frac{\lambda}{\left(\mu_{2}+\mu_{1}\right)^{2}}=\frac{3}{\mu_{2}+\mu_{1}}+\frac{\rho}{\mu_{2}+\mu_{1}}
$$

onde

$$
\rho=\frac{\lambda}{\mu_{2}+\mu_{1}}
$$

Demonstração: Lembrando-se que

$$
\begin{aligned}
& E\left(X_{2}\right)=\frac{1}{\mu_{2}} \\
& f(t)=\mu_{2} e^{-\mu_{2} t}, \\
& \phi_{X_{2}}(s)=\int_{0}^{\infty} e^{-s t} \mu_{2} e^{\mu_{2} t} d t=\frac{\mu_{2}}{\mu_{2}+s}, \\
& \frac{d}{d s} \phi_{X_{2}}\left(\mu_{1}\right)=-\frac{\mu_{2}}{\mu_{2}+s} .
\end{aligned}
$$


Então,

$$
\begin{aligned}
E(Y \mid 1) & =\left(1-\phi_{X_{2}}\left(\mu_{1}\right)\right) \frac{1}{\mu_{1}}\left(2+\frac{\lambda}{2 \mu_{1}}\right)+E\left(X_{2}\right) \phi_{X_{2}}\left(\mu_{1}\right)-\left.B \frac{d}{d s} \phi_{X_{2}}(s)\right|_{s=\mu_{1}}= \\
& =\left(1-\frac{\mu_{2}}{\mu_{2}+\mu_{1}}\right) \frac{1}{\mu_{1}}\left(2+\frac{\lambda}{2 \mu_{1}}\right)+\frac{1}{\mu_{2}}\left(\frac{\mu_{2}}{\mu_{2}+\mu_{1}}\right)-\frac{\lambda}{2}\left(\frac{1}{\mu_{2}}-\frac{1}{\mu_{1}}\right)\left(-\frac{\mu_{2}}{\left(\mu_{2}+\mu_{1}\right)^{2}}\right)= \\
& =\left(\frac{\mu_{1}}{\mu_{2}+\mu_{1}}\right) \frac{1}{\mu_{1}}\left(2+\frac{\lambda}{2 \mu_{1}}\right)+\frac{1}{\mu_{2}+\mu_{1}}+\frac{\lambda}{2}\left(\frac{\mu_{1}-\mu_{2}}{\mu_{2} \mu_{1}}\right) \frac{\mu_{2}}{\left(\mu_{1}+\mu_{2}\right)^{2}}= \\
& =\frac{3}{\mu_{2}+\mu_{1}}+\frac{\lambda}{2 \mu_{1}\left(\mu_{1}+\mu_{12}\right)}+\left(\frac{\mu_{1}-\mu_{2}}{2 \mu_{1}}\right) \frac{\lambda}{\left(\mu_{1}+\mu_{2}\right)^{2}}= \\
& =\frac{3}{\mu_{2}+\mu_{1}}+\frac{\rho}{2 \mu_{1}}+\frac{\mu_{1}-\mu_{2}}{2 \mu_{1}} \frac{\rho}{\mu_{1}+\mu_{2}}= \\
& =\frac{3}{\mu_{2}+\mu_{1}}+\frac{\rho}{\mu_{2}+\mu_{1}} .
\end{aligned}
$$

Assim, concluímos a prova do corolário.

Vamos agora calcular o valor de $E(Q \backslash 2)$ da equação (5.1). Temos três casos possíveis: no primeiro caso, $C_{1}$ termina o serviço antes que $C_{2}$ chegue ao sistema, assim o cliente esperto seleciona a fila 2, conforme a Figura 5.6.

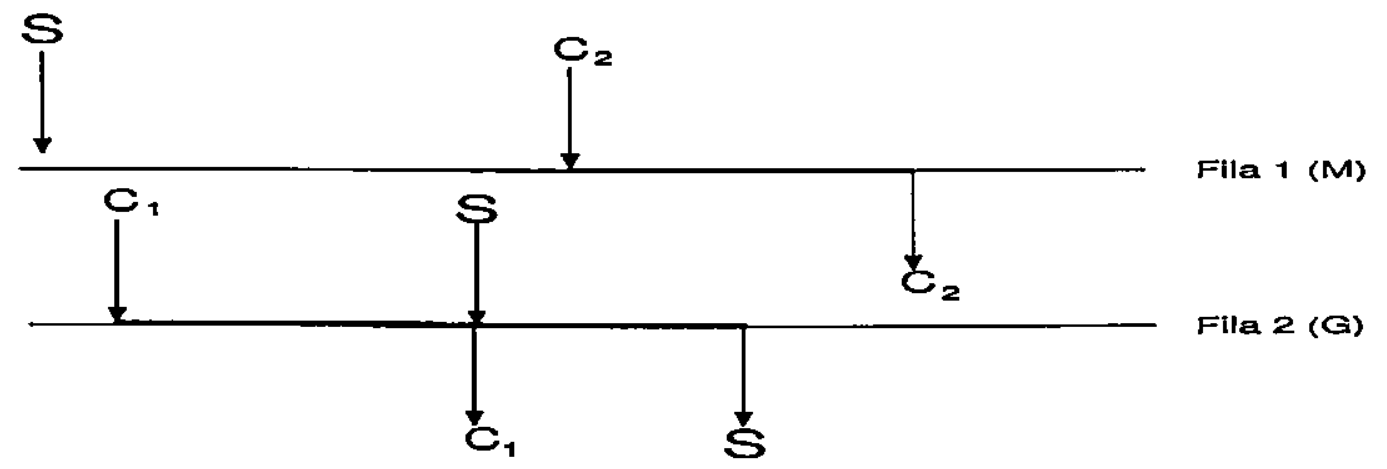

Figura 5.6: Uma realização onde $C_{1}$ termina o serviço antes da chegada de $C_{2}$

No segundo caso ambos os servidores estão ocupados, portanto os novos clientes que 
chegam ao sistema escolhem a fila mais curta. Se ambas as filas têm o mesmo comprimento, a escolha é aleatória. Se $C_{1}$ termina o serviço antes de $C_{2}$, então $S$ seleciona a fila 2, conforme a Figura 5.7.

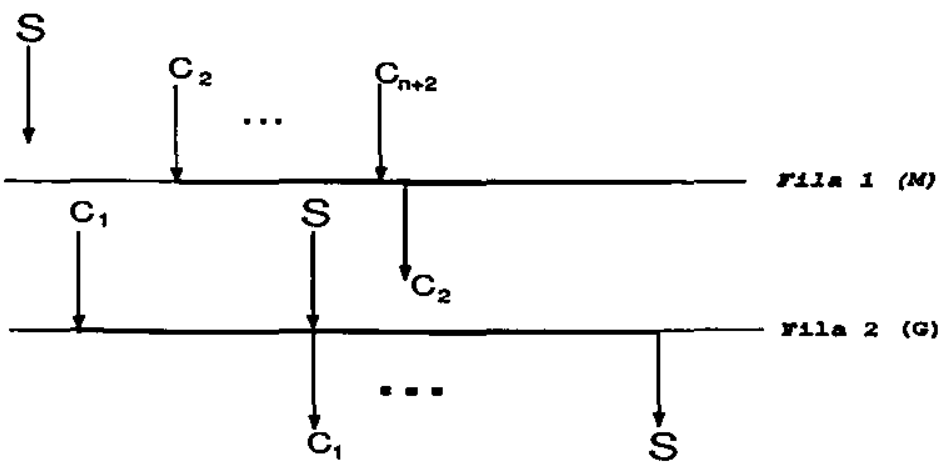

Figura 5.7: Uma realização onde $C_{1}$ termina o serviço antes do serviço de $C_{2}$

No terceiro caso, quando $C_{1}$ termina o serviço após o serviço do $C_{2}$, então $S$ seleciona a fila 1, conforme a Figura 5.8 .

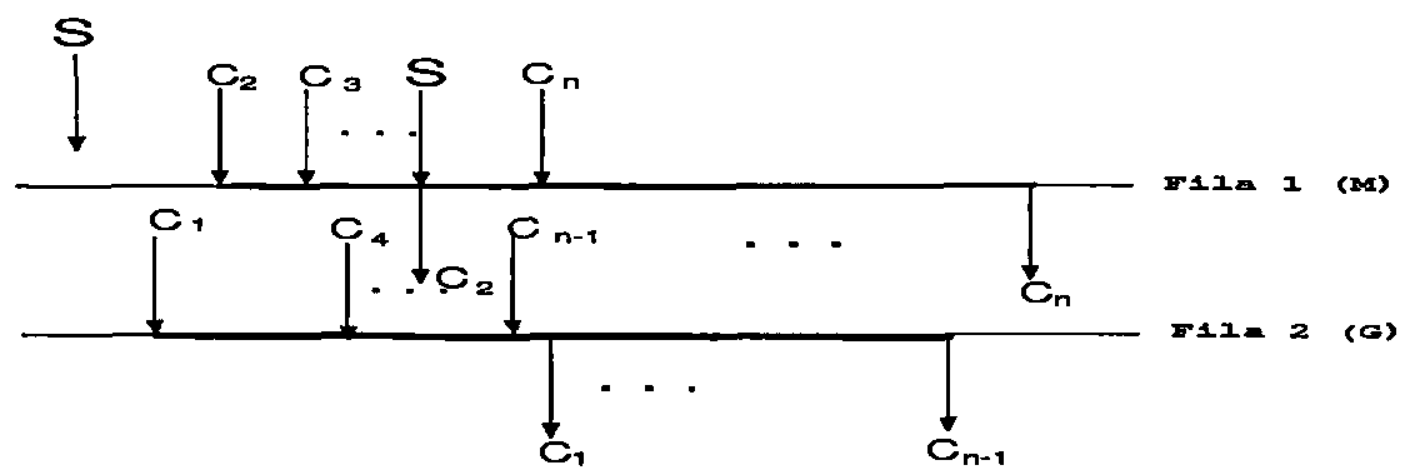

Figura 5.8: Representação de uma realizaçāo com serviço nos dois servidores

Consideremos o seguinte lema.

Lema 5.3.2. Sejam $\lambda \neq \mu_{1}, \quad A$ e $B$ definidos como no Lema 5.3.1. O tempo médio medido a partir do início do serviço de $C_{1}$ na fila 2, até $S$ completar o serviço, é dado por:

$$
\begin{aligned}
E(Q \mid 2) & =\left(A-\frac{1}{\lambda}\right)\left(1-\phi_{X_{2}}(\lambda)\right)+\left(\frac{\lambda}{\lambda-\mu_{1}}\right)\left[E\left(X_{2}\right)-A-\frac{B}{\lambda-\mu_{1}}\right]\left(\phi_{X_{2}}\left(\mu_{1}\right)-\phi_{X_{2}}(\lambda)\right) \\
& +E\left(X_{2}\right) \phi_{X_{2}}(\lambda)-\left.\frac{B \lambda}{\lambda-\mu_{1}} \frac{d}{d s}\left(\phi_{X_{2}}(s)\right)\right|_{s=\mu_{1}} .
\end{aligned}
$$


Demonstraçāo: Para provarmos o Lema 5.3.2 devemos determinar o tempo médio de permanência do cliente esperto para os três casos e somar, ou seja,

$$
\begin{aligned}
E(Q \mid 2)= & E\left(Q * I\left(T>X_{2}\right) \mid 2\right)+E\left(Q * I\left(T+X_{1}<X_{2}\right) \mid 2\right) \\
& +E\left(Q * I\left(T<X_{2}<T+X_{1}\right) \mid 2\right)
\end{aligned}
$$

onde

- T: a variável aleatória que mede o tempo entre o instante em que $C_{1}$ começa o serviço, até a próxima chegada;

- $Q * I\left(T>X_{1}\right)$ : indicando que $S$ seleciona a fila 2 e $C_{1}$ terminou o serviço antes de nova chegada;

- $Q * I\left(T+X_{1}<X_{2}\right)$ : indicando que $S$ seleciona a fila 1 , pois o cliente que chegou após $C_{1}$ terminou o serviço antes deste;

- $Q * I\left(T<X_{2}<T+X_{2}\right)$ : indicando que $S$ seleciona a fila 2 , pois o cliente que chegou após $C_{1}$ terminou o serviço depois deste.

No primeiro caso temos

$$
\begin{aligned}
E\left(Q * I\left(T>X_{2}\right) \mid 2\right) & =\int_{0}^{\infty}\left(t+E\left(X_{2}\right)\right) e^{-\lambda t} d P\left[X_{2} \leq t\right]= \\
& =\int_{0}^{\infty} t e^{-\lambda t} d P\left[X_{2} \leq t\right]+E\left(X_{2}\right) \int_{0}^{\infty} e^{-\lambda t} d P\left[X_{2} \leq t\right]= \\
& =-\left.\frac{d}{d s} \phi_{X_{2}}(s)\right|_{s=\lambda}+E\left(X_{2}\right) \phi_{X_{2}}(\lambda) .
\end{aligned}
$$

No segundo caso temos

$$
E\left(Q * I\left(T+X_{1}<X_{2}\right) \mid 2\right)=\int_{0}^{\infty} \int_{\tau=0}^{t} \int_{x=0}^{t-\tau}\left(\tau+x+\left(\frac{\lambda x}{2}+1\right) \frac{1}{\mu_{1}}\right) \mu_{1} e^{-\mu_{1} x} d x \lambda e^{-\lambda \tau} d \tau d P\left[X_{2} \leq t\right]
$$

Desenvolvendo o segundo membro desta equação, primeiro integrando em relação $x$, $\mathrm{e}$ 
80

Observando Filas Paralelas com Serviço Geral

fazendo $I_{1}$ como sendo o termo interno da integral, temos

$$
\begin{aligned}
I_{1} & =\int_{0}^{t-\tau}\left(\tau+x+\left(\frac{\lambda x}{2}+1\right) \frac{1}{\mu_{1}}\right) \mu_{1} e^{-\mu_{1} x} d x= \\
& =\left(\tau+\frac{1}{\mu_{1}}\right) \mu_{1} \int_{0}^{t-\tau} e^{-\mu_{1} x} d x+\left(1+\frac{\lambda}{2 \mu_{1}}\right) \mu_{1} \int_{0}^{t-\tau} x e^{-\mu_{1} x} d x= \\
& =\left(\tau+\frac{1}{\mu_{1}}\right) \mu_{1} \frac{1}{\mu_{1}}\left(1-e^{-\mu_{1}(t-\tau)}\right)+\left(1+\frac{\lambda}{2 \mu_{1}}\right) \mu_{1}\left(\frac{1}{\mu_{1}^{2}}\left(1-e^{-\mu_{1}(t-\tau)}\right)-\frac{(t-\tau)}{\mu_{1}} e^{-\mu_{1}(t-\tau)}\right)= \\
& =\left(\tau+\frac{1}{\mu_{1}}\right) \frac{1}{\mu_{1}}\left(1-e^{-\mu_{1}(t-\tau)}\right)+\left(1+\frac{\lambda}{2 \mu_{1}}\right)\left(\frac{1}{\mu_{1}}\left(1-e^{-\mu_{1}(t-\tau)}\right)-(t-\tau) e^{-\mu_{1}(t-\tau)}\right) .
\end{aligned}
$$

Portanto, temos

$$
\begin{aligned}
E\left(Q * I\left(T+X_{1}<X_{2}\right) \mid 2\right) & =\int_{0}^{\infty} \int_{\tau=0}^{t}\left(\tau+\frac{1}{\mu_{1}}\right)\left(1-e^{-\mu_{1}(t-\tau)}\right)+\left(1+\frac{\lambda}{2 \mu_{1}}\right)\left(\frac{1}{\mu_{1}}\left(1-e^{-\mu_{1}(t-\tau)}\right)\right. \\
& \left.-(t-\tau) e^{-\mu_{1}(t-\tau)}\right) \lambda e^{-\lambda \tau} d \tau d P\left[X_{2} \leq t\right]
\end{aligned}
$$

Integrando em relação $\tau$ e fazendo $I_{2}$ como sendo a primeira parcela do termo interno da integral acima, temos

$$
\begin{aligned}
I_{2} & =\int_{0}^{t}\left(\tau+\frac{1}{\mu_{1}}\right)\left(1-e^{-\mu_{1}(t-\tau)}\right) \lambda e^{-\lambda \tau} d \tau= \\
& =\lambda\left[\int_{0}^{t} \tau e^{-\lambda \tau} d \tau-e^{-\mu_{1} t} \int_{0}^{t} \tau e^{-\left(\lambda-\mu_{1}\right) \tau} d \tau+\frac{1}{\mu_{1}} \int_{0}^{t} e^{-\lambda \tau} d \tau-\frac{e^{-\mu_{1} t}}{\mu_{1}} \int_{0}^{t} e^{-\left(\lambda-\mu_{1}\right)} \tau d t\right]= \\
& =\lambda\left[\frac{1}{\lambda^{2}}\left(1-e^{-\lambda t}\right)-\frac{t}{\lambda}-e^{\mu_{1} t}\left(\frac{1}{\left(\lambda-\mu_{1}\right)^{2}}\left(1-e^{-(\lambda-\mu) t}\right)-\frac{t}{\lambda-\mu_{1}} e^{-\left(\lambda-\mu_{1}\right) t}+\frac{1}{\mu_{1}} \frac{1}{\lambda}\left(1-e^{-\lambda t}\right)\right)\right]= \\
& =\frac{1}{\lambda}\left(1-e^{-\lambda t}\right)-t e^{-\lambda t}-\frac{\lambda}{\left(\lambda-\mu_{1}\right)^{2}}\left(e^{-\mu_{1} t}-e^{-\lambda t}\right)+\frac{\lambda t}{\lambda-\mu_{1}} e^{-\lambda t}+\frac{1}{\mu_{1}}\left(1-e^{-\lambda t}\right) \\
& -\frac{\lambda}{\lambda-\mu_{1}} \frac{1}{\mu_{1}}\left(e^{-\mu_{1} t}-e^{-\lambda t}\right)= \\
& =\left(\frac{1}{\lambda}+\frac{1}{\mu_{1}}\right)\left(1-e^{-\lambda t}\right)+\frac{t \mu_{1}}{\lambda-\mu_{1}} e^{-\lambda t}-\frac{\lambda^{2}}{\left(\lambda-\mu_{1}\right)^{2}} \frac{1}{\mu_{1}}\left(e^{-\mu_{1} t}-e^{-\lambda t}\right) .
\end{aligned}
$$


Por outro lado, fazendo $I_{3}$ como sendo a segunda parcela da parte interna da integral da equação (5.5), temos

$$
\begin{aligned}
I_{3} & =\int_{0}^{t}\left(1+\frac{\lambda}{2 \mu_{1}}\right)\left(\frac{1}{\mu_{1}}\left(1-e^{-\mu_{1}(t-\tau)}\right)-(t-\tau) e^{-\mu_{1}(t-\tau)}\right) \lambda e^{-\lambda \tau} d \tau= \\
& =\left(1+\frac{\lambda}{2 \mu_{1}}\right) \lambda\left[\frac{1}{\mu_{1}} \int_{0}^{t} e^{-\lambda \tau} d \tau-\frac{1}{\mu_{1}} e^{-\mu_{1} t} \int_{0}^{t} e^{-\left(\lambda-\mu_{1}\right)} \tau d \tau-e^{-\mu_{1} t} t \int_{0}^{t} \tau e^{-\left(\lambda-\mu_{1}\right) \tau} d \tau\right. \\
& \left.+e^{-\mu_{1} t} \int_{0}^{t} \tau e^{-\left(\lambda-\mu_{1}\right) \tau} d \tau\right]= \\
& =\left(1+\frac{\lambda}{2 \mu_{1}}\right) \lambda\left[\frac{1}{\mu_{1}} \frac{1}{\lambda}\left(1-e^{-\lambda t}\right)-\frac{e^{-\mu_{1} t}}{\mu_{1}} \frac{1}{\lambda-\mu_{1}}\left(1-e^{-\left(\lambda-\mu_{1}\right) t}\right)-t e^{-\mu_{1} t} \frac{1}{\lambda-\mu_{1}}\left(1-e^{-\left(\lambda-\mu_{1}\right) t}\right)\right. \\
& \left.+e^{-\mu_{1} t}\left(\frac{1}{\left(\lambda-\mu_{1}\right)^{2}}\right)\left(1-e^{-\left(\lambda-\mu_{1}\right) t}\right)-\frac{t}{\lambda-\mu_{1}} e^{-\left(\lambda-\mu_{1}\right) t}\right]= \\
= & \left(1+\frac{\lambda}{2 \mu_{1}}\right)\left[\frac{1-e^{-\lambda t}}{\mu_{1}}-\frac{\lambda}{\mu_{1}}\left(\frac{e^{-\mu_{1} t}-e^{-\lambda t}}{\lambda-\mu_{1}}\right)-\frac{t \lambda}{\lambda-\mu_{1}} e^{-\mu_{1} t}+\frac{\lambda}{\left(\lambda-\mu_{1}\right)^{2}}\left(e^{-\mu_{1} t}-e^{-\lambda t}\right)\right]
\end{aligned}
$$

Logo, obtemos

$$
\begin{aligned}
& E\left(Q * I\left(T+X_{1}<X_{2}\right) \mid 2\right)=\int_{0}^{\infty}\left(I_{2}+I_{3}\right) d P\left[X_{2} \leq t\right]= \\
& =\int_{0}^{\infty}\left\{\left(\frac{1}{\lambda}+\frac{1}{\mu_{1}}\left(2+\frac{\lambda}{2 \mu_{1}}\right)\right)\left(1-e^{-\lambda t}\right)+\frac{t \mu_{1}}{\lambda-\mu_{1}} e^{-\lambda t}-\left(1+\frac{\lambda}{2 \mu_{1}}\right) \frac{t \lambda}{\lambda-\mu_{1}} e^{-\mu_{1} t}-\left[\frac{\lambda^{2}}{\left(\lambda-\mu_{1}\right)^{2} \mu_{1}}+\right.\right. \\
& \left.\left.\left(1+\frac{\lambda}{2 \mu_{1}}\right)\left(\frac{\lambda}{\mu_{1}\left(\lambda-\mu_{1}\right)^{2}}-\frac{\lambda}{\left(\lambda-\mu_{1}\right)^{2}}\right)\right]\left(e^{-\mu_{1} t}-e^{-\lambda t}\right)\right\} d P\left[X_{2} \leq t\right]= \\
& =\int_{0}^{\infty}\left\{\left(\frac{1}{\lambda}+A\right)\left(1-e^{-\lambda t}\right)+\frac{t \mu_{1}}{\lambda-\mu_{1}} e^{-\lambda t}-\left(1+\frac{\lambda}{2 \mu_{1}}\right) \frac{t \lambda}{\lambda-\mu_{1}} e^{-\mu_{1} t}-\left[\frac{\lambda^{2}}{\left(\lambda-\mu_{1}\right)^{2} \mu_{1}}+\right.\right. \\
& \left.\left.\left.\left(1+\frac{\lambda}{2 \mu_{1}}\right)\left(\frac{\lambda}{\mu_{1}\left(\lambda-\mu_{1}\right)^{2}}-\frac{\lambda}{\left(\lambda-\mu_{1}\right)^{2}}\right)\right]\right\}\left(e^{-\mu_{1} t}-e^{-\lambda t}\right)\right\} d P\left[X_{2} \leq t\right]= \\
& =\left(\frac{1}{\lambda}+A\right)\left(1-\phi_{X_{2}}(\lambda)\right)-\frac{\mu_{1}}{\lambda-\mu_{1}}\left(\left.\frac{d}{d s} \phi_{X_{2}}(s)\right|_{s=\lambda}\right)+\left(1+\frac{\lambda}{2 \mu_{1}}\right) \frac{\lambda}{\lambda-\mu_{1}}\left(-\left.\frac{d}{d s} \phi_{X_{2}}(s)\right|_{s=\mu_{1}}\right)- \\
& {\left[\frac{\lambda^{2}}{\left(\lambda-\mu_{1}\right)^{2} \mu_{1}}+\left(1+\frac{\lambda}{2 \mu_{1}}\right)\left(\frac{\lambda}{\mu_{1}\left(\lambda-\mu_{1}\right)}-\frac{\lambda}{\left(\lambda-\mu_{1}\right)^{2}}\right)\right]\left(\phi_{X_{2}}\left(\mu_{1}\right)-\phi_{X_{2}}(\lambda)\right) \text {. }}
\end{aligned}
$$


No terceiro caso temos

$$
\begin{aligned}
& E\left(Q * I\left(T<X_{2}<T+X_{1}\right) \mid 2\right)=\int_{0}^{\infty} \int_{0}^{t} e^{-\mu_{1}(t-\tau)}\left(t+\left(\frac{\lambda(t-\tau)}{2}+1\right) E\left(X_{2}\right)\right) \lambda e^{-\lambda \tau} d \tau \\
& d P\left[X_{2} \leq t\right]= \\
& =\int_{0}^{\infty}\left\{\left(1+\frac{\lambda E\left(X_{2}\right)}{2}\right) t \lambda \int_{0}^{t} e^{-\mu_{1} t} e^{-\left(\lambda-\mu_{1}\right) \tau} d \tau\right\}+E\left(X_{2}\right) \lambda e^{-\mu_{1} t} \int_{0}^{t} e^{-\left(\lambda-\mu_{1}\right) t} d \tau- \\
& \frac{\lambda^{2} E\left(X_{2}\right)}{2} e^{-\mu_{1} t} \int_{0}^{t} \tau e^{-\left(\lambda-\mu_{1}\right) \tau} d \tau= \\
& =\int_{0}^{\infty}\left\{\left(1+\frac{\lambda E\left(X_{2}\right)}{2}\right) t \lambda e^{-\mu_{1} t} \frac{1}{\lambda-\mu_{1}}\left(1-e^{-\left(\lambda-\mu_{1}\right) t}\right)+E\left(X_{2}\right) e^{-\mu_{1} t} \frac{\lambda}{\lambda-\mu_{1}}\left(1-e^{-\left(\lambda-\mu_{1}\right) t}\right)-\right. \\
& \left.\frac{\lambda^{2} E\left(X_{2}\right)}{2} e^{-\mu_{1} t}\left[\frac{1}{\left(\lambda-\mu_{1}\right)^{2}}\left(1-e^{-\left(\lambda-\mu_{1}\right) t}\right)-\frac{t}{\lambda-\mu_{1}} e^{-\left(\lambda-\mu_{1}\right) t}\right]\right\} d P\left[X_{2} \leq t\right]= \\
& \frac{\lambda^{2} E\left(X_{2}\right)}{2}\left[\frac{\left.\phi_{X_{2}}\left(\mu_{1}\right)-\phi_{X_{2}}(\lambda)\right)}{\left(\lambda-\mu_{1}\right)^{2}}+\left.\frac{1}{\lambda-\mu_{1}} \frac{d}{d s} \phi_{X_{2}}(s)\right|_{s=\lambda}\right] \\
& =\left(1+\frac{\lambda E\left(X_{2}\right)}{2}\right) \frac{\lambda}{\lambda-\mu_{1}}\left[-\left.\frac{d}{d s} \phi_{X_{2}}(s)\right|_{s=\mu_{1}}+\left.\frac{d}{d s} \phi_{X_{2}}(s)\right|_{s=\lambda}\right]+\frac{\lambda}{\lambda-\mu_{1}} E\left(X_{2}\right)\left(\phi_{X_{2}}\left(\mu_{1}\right)-\phi_{X_{2}}(\lambda)\right)-
\end{aligned}
$$

Somando-se os resultados dos três casos, obtemos

$$
\begin{aligned}
& E(Q \mid 2)=-\left.\frac{d}{d s} \phi_{X_{2}}(s)\right|_{s=\lambda}+E\left(X_{2}\right) \phi_{X_{2}}(\lambda)+\left(\frac{1}{\lambda}+A\right)\left(1-\phi_{X_{2}}(\lambda)\right)-\frac{\mu_{1}}{\lambda-\mu_{1}}\left(\left.\frac{d}{d s} \phi_{X_{2}}(s)\right|_{s=\lambda}\right) \\
&+\left(1+\frac{\lambda}{2 \mu_{1}}\right) \frac{\lambda}{\lambda-\mu_{1}}\left(-\left.\frac{d}{d s} \phi_{X_{2}}(s)\right|_{s=\mu_{1}}\right)-\left[\frac{\lambda^{2}}{\left(\lambda-\mu_{1}\right)^{2} \mu_{1}}+\left(1+\frac{\lambda}{2 \mu_{1}}\right)\left(\frac{\lambda}{\mu_{1}\left(\lambda-\mu_{1}\right)}\right.\right. \\
&\left.\left.-\frac{\lambda}{\left(\lambda-\mu_{1}\right)^{2}}\right)\right]\left(\phi_{X_{2}}\left(\mu_{1}\right)-\phi_{X_{2}}(\lambda)\right)+\left(1+\frac{\lambda E\left(X_{2}\right)}{2}\right) \frac{\lambda}{\lambda-\mu_{1}}\left[-\left.\frac{d}{d s} \phi_{X_{2}}(s)\right|_{s=\mu_{1}}\right. \\
&\left.+\left.\frac{d}{d s} \phi_{X_{2}}(s)\right|_{s=\lambda}\right]+\frac{\lambda}{\lambda-\mu_{1}} E\left(X_{2}\right)\left(\phi_{X_{2}}\left(\mu_{1}\right)-\phi_{X_{2}}(\lambda)-\frac{\lambda^{2} E\left(X_{2}\right)}{2}\left[\frac{\phi_{X_{2}}\left(\mu_{1}\right)-\phi_{X_{2}}(\lambda)}{\left(\lambda-\mu_{1}\right)^{2}}\right.\right.
\end{aligned}
$$




$$
\left.+\left.\frac{1}{\lambda-\mu_{1}} \frac{d}{d s} \phi_{X_{2}}(s)\right|_{s=\lambda}\right]
$$

Logo,

$$
\begin{aligned}
E(Q \mid 2)= & \left(\frac{1}{\lambda}+A\right)\left(1-\phi_{X_{2}}(\lambda)\right)+E\left(X_{2}\right) \phi_{X_{2}}(\lambda)-\frac{B \lambda}{\lambda-\mu_{1}}\left(\left.\frac{d}{d s} \phi_{X_{2}}(s)\right|_{s=\mu_{1}}\right)+\frac{\lambda}{\lambda-\mu_{1}}\left(E\left(X_{2}\right)\right. \\
& \left.-A-\frac{B}{\lambda-\mu_{1}}\right)\left(\phi_{X_{2}}\left(\mu_{1}\right)-\phi_{X_{2}}(\lambda)\right)
\end{aligned}
$$

o que completa a prova do Lema 5.3.2.

\subsection{Análise do Modelo com Servidores Não-exponenciais}

Consideremos o modelo $G /\left(\left(H_{2}+G\right) / 1\right)^{2}$, onde $H_{2}$ representa uma distribuição Hiperexponencial de ordem 2 que tem função densidade e transformada de Laplace-Stieltjes dadas, respectivamente, por

$$
f(t)=\sum_{i=1}^{2} p_{i} \mu_{i} e^{-\mu_{i} t}, t \geq 0 \quad \text { e } \quad \phi(s)=\sum_{i=1}^{2} p_{i} \frac{\mu_{i}}{s+\mu_{i}}
$$

onde $\sum_{i=1}^{2} p_{i}=1$

Para esta distribuição temos

$$
E[X]=\sum_{i=1}^{2} \frac{p_{i}}{\mu_{i}}, \quad E\left[X^{2}\right]=2 \sum_{i=1}^{2} \frac{p_{i}}{\mu_{i}{ }^{2}} \quad \text { e } \quad \operatorname{Var}[X]=E\left[X^{2}\right]-(E[X])^{2}
$$

Os parâmetros da distribuição Hiperexponencial de ordem 2 não são unicamente ajustados pelos primeiros dois momentos. Diz-se que uma distribuição $\mathrm{H}_{2}$ tem médias balanceadas se a normalização é igual a $\frac{p_{1}}{\mu_{1}}=\frac{p_{2}}{\mu_{2}}$.

Os parâmetros da densidade de $\mathrm{H}_{2}$ com médias balanceadas ajustadas pelos primeiros momentos são dados por

$$
p_{1}=\frac{1}{2}\left(1+\sqrt{\frac{c_{x}^{2}+1}{c_{x}^{2}+1}}\right), \quad p_{2}=1-p_{1} \quad, \quad \mu_{1}=\frac{2 p_{1}}{E[X]} \text { e } \mu_{2}=\frac{2 p_{2}}{E[X]},
$$


onde $c_{x}^{2}$ é quadrado do coeficiente de variação dos tempos de serviço. Para maiores detalhes sobre o ajuste de distribuições $H_{2}$, ver Tijms(86).

Vamos analisar o tempo médio desde o instante em que $C_{1}$ começa o serviço até que $S$ conclua o serviço. Temos dois casos possíveis: no primeiro caso, $C_{1}$ seleciona a fila 1 e o tempo médio desde que $C_{1}$ começa o serviço até a próxima ocorrência de eventos, ou seja, uma chegada ou conclusão de serviço, o qual designaremos por $E(R \mid 1)$, é dado por

$$
E(R \mid 1)=\frac{1}{\lambda+\mu_{1}} p_{1}+\frac{1}{\lambda+\mu_{2}} p_{2}=\sum_{i=1}^{2} \frac{1}{\lambda+\mu_{i}} p_{i}
$$

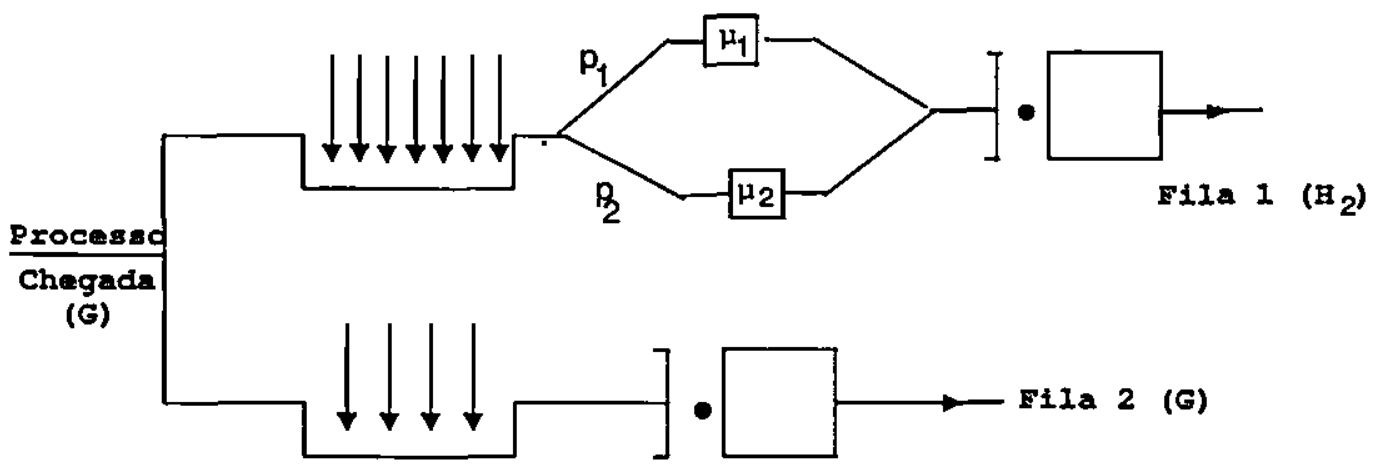

Figura 5.9: Uma representação do modelo $G /\left(\left(H_{2}+G\right) / 1\right)^{2}$

A probabilidade de que a próxima ocorrência seja uma conclusão de serviço e de $C_{1}$ selecionar a fila 1 é dada por

$$
\begin{aligned}
P\left(A_{1} \mid 1\right) & =\int_{0}^{\infty} P\left(\text { tempo até a chegada de } C_{2}>t\right) P\left(\text { serviço } C_{1}<t\right) d t= \\
& =\int_{0}^{\infty} e^{-\lambda t} \sum_{i=1}^{2} p_{i} \mu_{i} e^{-\mu_{i} t} d t=\sum_{i=1}^{2} \frac{p_{i} \mu_{i}}{\lambda+\mu_{i}}
\end{aligned}
$$

Logo,

$$
\begin{aligned}
E(Q \mid 1) & =E(R \mid 1)+P\left(A_{1} \mid 1\right) E\left(X_{1}\right)+\left(1-P\left(A_{1} \mid 1\right)\right) E\left(Y_{i} \mid 1\right)= \\
& =\sum_{i=1}^{2} \frac{1}{\lambda+\mu_{i}} p_{i}+\sum_{i=1}^{2} \frac{\mu_{i}}{\mu_{i}+\lambda} E\left(X_{1}\right)+\sum_{i=1}^{2} \frac{\lambda}{\mu_{i}+\lambda} E\left(Y_{1} \mid 1\right)= \\
& =\sum_{i=1}^{2} p_{i}\left(\frac{1}{\lambda+\mu_{i}}+\left(\frac{\mu_{i}}{\mu_{i}+\lambda}\right) E\left(X_{1}\right)+\frac{\lambda}{\mu_{i}+\lambda} E\left(Y_{i} \mid 1\right)\right) E(Q \mid 1) .
\end{aligned}
$$


O tempo médio a partir do instante em que ambos servidores estiveram ocupados, até que $S$ complete o serviço, desde que $C_{1}$ seleciona a fila 1 , é obtido analisando dois casos: no primeiro caso, o tempo residual de $C_{1}$ é menor do que o tempo de serviço de $C_{2}$; no segundo caso, o tempo residual de $C_{1}$ é maior do que o tempo de serviço de $C_{2}$. Para calcularmos o tempo médio, utilizamos o mesmo procedimento feito para o Lema 5.3.1. Temos

$$
E(Y \mid 1)=E\left(Y * I\left(X_{1}^{\prime}<X_{2}\right) \mid 1\right)+E\left(Y * I\left(X_{1}^{\prime} \geq X_{2}\right) \mid 1\right)
$$

Vamos primeiro calcular o primeiro termo que é do segundo membro:

$$
\begin{aligned}
& E\left(Y * I\left(X_{1}^{\prime}<X_{2}\right) \mid 1\right)=\int_{0}^{\infty} \int_{\tau=0}^{t}\left(\tau+\left(\frac{\lambda \tau}{2}+1\right) E\left(X_{1}\right)\right) \mu_{i} e^{-\mu_{i} \tau} d \tau d P\left[X_{2} \leq t\right]= \\
& =\int_{0}^{\infty}\left\{\left(1+\frac{\lambda E\left(X_{1}\right)}{2}\right) \int_{0}^{t} \tau \mu_{i} e^{-\mu_{i} \tau} d \tau+E\left(X_{1}\right) \int_{0}^{t} \mu_{i} e^{-\mu_{i} t} d \tau\right\} d P\left[X_{2} \leq t\right]= \\
& =\int_{0}^{\infty}\left\{\left(1+\frac{\lambda E\left(X_{1}\right)}{2}\right) \mu_{i}\left[\frac{1}{\mu_{i}{ }^{2}}\left(1-e^{-\mu_{i} t}\right)-\frac{t}{\mu_{i}} e^{-\mu_{i} t}\right]+E\left(X_{1}\right)\left(1-e^{-\mu_{i} t}\right)\right\} d P\left[X_{2} \leq t\right]= \\
& =\int_{0}^{\infty}\left\{\left(1+\frac{\lambda E\left(X_{1}\right)}{2}\right) \mu_{i}\left[\frac{1}{\mu_{i}{ }^{2}}\left(1-e^{-\mu_{i} t}\right)-\frac{t}{\mu_{i}} e^{-\mu_{i} t}\right]+E\left(X_{1}\right)\left(1-e^{-\mu_{i} t}\right)\right\} d P\left[X_{2} \leq t\right]= \\
& =\int_{0}^{\infty}\left\{\left(\frac{1}{\mu_{i}}+\frac{\lambda E\left(X_{1}\right)}{2 \mu_{1}}+E\left(X_{1}\right)\right)\left(1-e^{-\mu_{i} t}\right)-t\left(1+\frac{\lambda E\left(X_{1}\right)}{2}\right) e^{-\mu_{i} t}\right\} d P\left[X_{2} \leq t\right] .
\end{aligned}
$$

Integrando em relaçāo a $P\left[X_{2} \leq t\right]$, temos

$$
\begin{aligned}
E\left(Y * I\left(X_{1}^{\prime}<X_{2}\right) \mid 1\right)= & \left(\frac{1}{\mu_{i}}+E\left(X_{1}\right)\left(1+\frac{\lambda}{2 \mu_{1}}\right)\right)\left(1-\phi_{X_{2}}\left(\mu_{i}\right)\right)- \\
& \left(1+\frac{\lambda E\left(X_{1}\right)}{2}\right)\left(-\left.\frac{d}{d s} \phi_{X_{2}}(s)\right|_{s}=\mu_{i}\right) .
\end{aligned}
$$

Por outro lado, o segundo termo do segundo membro da equação (5.5) é dado por

$$
\begin{aligned}
E\left(Y * I\left(X_{1}^{\prime} \geq X_{2}\right) \mid 1\right) & =\int_{0}^{\infty} e^{-\mu_{i} t}\left(t+\left(\frac{\lambda t}{2}+1\right) E\left(X_{2}\right)\right) d P\left[X_{2} \leq t\right]= \\
& =\left(1+\frac{\lambda E\left(X_{2}\right)}{2}\right) \int_{0}^{\infty} t e^{-\mu_{i} t} d P\left[X_{2} \leq t\right]+E\left(X_{2}\right) \int_{0}^{\infty} e^{-\mu_{i} t} d P\left[X_{2} \leq t\right]
\end{aligned}
$$




$$
E\left(Y * I\left(X_{1}^{\prime} \geq X_{2}\right) \mid 1\right)=\left(1+\frac{\lambda E\left(X_{2}\right)}{2}\right)\left(-\left.\frac{d}{d s} \phi_{X_{2}}(s)\right|_{s}=\mu_{1}\right)+E\left(X_{2}\right) \phi_{X_{2}}\left(\mu_{i}\right)
$$

Assim, somando-se os dois termos obtemos

$$
\begin{aligned}
E(Y \mid 1)= & \left(\frac{1}{\mu_{i}}+E\left(X_{1}\right)\left(1+\frac{\lambda}{2 \mu_{1}}\right)\right)\left(1-\phi_{X_{2}}\left(\mu_{i}\right)\right)+E\left(X_{2}\right) \phi_{X_{2}}\left(\mu_{i}\right)- \\
& \frac{\lambda}{2}\left(E\left(X_{2}\right)-E\left(X_{1}\right)\right) \frac{d}{d s} \phi_{\left.X_{2}(s)\right|_{s=\mu_{i}}} .
\end{aligned}
$$

No segundo caso, $C_{1}$ seleciona a fila 2 . $O$ tempo médio medido a partir do início do serviço de $C_{1}$ até $S$ completar o serviço é igual a

$$
E(Q \mid 2)=E\left(Q * I\left(T+X_{1}<X_{2}\right) \mid 2\right)+E\left(Q * I\left(T<X_{2}<T+X_{1}\right) \mid 2\right) .
$$

Vamos primeiro calcular o primeiro termo que é igual a

$$
\begin{aligned}
E\left(Q * I\left(T+X_{1}<X_{2}\right) \mid 2\right)= & \int_{0}^{\infty} \int_{0}^{t} \int_{x=0}^{t-\tau}\left[\tau+x+\left(\frac{\lambda x}{2}+1\right) E\left(X_{2}\right)\right] \sum_{i=0}^{2} p_{i} \mu_{i} e^{-\mu_{i} x} d x \lambda e^{-\lambda \tau} \\
& d \tau d P\left[X_{2} \leq t\right] .
\end{aligned}
$$

Integrando em relação a $x$ e fazendo $I_{4}$ igual à primeira parcela do termo interno da integral acima, temos

$$
\begin{aligned}
I_{4}= & \int_{x=0}^{t-\tau}\left[\tau+x+\left(\frac{\lambda x}{2}+1\right) E\left(X_{2}\right)\right] \sum_{i=1}^{2} p_{i} \mu_{i} e^{-\mu_{i} x} d x= \\
= & \int_{x=0}^{t-\tau}\left(\tau+E\left(X_{1}\right)\right) \sum_{i=1}^{2} p_{i} \mu_{i} e^{-\mu_{i} x} d x+\int_{x=1}^{t-\tau}\left(1+\frac{\lambda E\left(X_{1}\right)}{2}\right) x \sum_{i=1}^{2} p_{i} \mu_{i} e^{-\mu_{i} x} d x= \\
= & \left(\tau+E\left(X_{1}\right)\right) \sum_{i=1}^{2} p_{i}\left(1-e^{-\mu_{i}(t-\tau)}\right)+\left(1+\frac{\lambda E\left(X_{1}\right)}{2}\right) \sum_{i=1}^{2} \frac{p_{i}}{\mu_{i}}\left(1-e^{-\mu_{i}(t-\tau)}\right)- \\
& \left(1+\frac{\lambda E\left(X_{1}\right)}{2}\right) \sum_{i=1}^{2} p_{i}(t-\tau) e^{-\mu_{i}(t-\tau)} .
\end{aligned}
$$


Logo,

$$
E\left(Q * I\left(T+X_{1}<X_{2}\right) \mid 2\right)=\int_{0}^{\infty} \int_{0}^{t} I_{4} \lambda e^{-\lambda \tau} d \tau d P\left[X_{2} \leq t\right]
$$

Substituindo-se o valor de $I_{4}$ na equação 5.6, integrando-se em $\tau$ e fazendo-se de $I_{41}$ igual à primeira parcela do termo interno da integral acima, temos

$$
\begin{aligned}
I_{41}= & \int_{0}^{t}\left(\tau+E\left(X_{1}\right)\right) \sum_{i=1}^{2} p_{i}\left(1-e^{-\mu_{i}(t-\tau)}\right) \lambda e^{-\lambda \tau} d \tau= \\
= & \lambda \sum_{i=1}^{2} p_{i}\left[\int_{0}^{t}\left(\tau+E\left(X_{1}\right)\right)\left(e^{-\lambda \tau}-e^{-\mu_{i} t} e^{-\left(\lambda-\mu_{i}\right) \tau}\right)\right] d \tau= \\
= & \lambda \sum_{i=1}^{2} p_{i}\left[\int_{0}^{t} \tau e^{-\lambda \tau} d \tau-e^{-\mu_{i} t} \int_{0}^{t} \tau e^{-\left(\lambda-\mu_{i}\right) \tau} d \tau+E\left(X_{1}\right) e^{-\mu_{i} t} \int_{0}^{t} e^{-\left(\lambda-\mu_{i}\right) \tau} d \tau\right]= \\
= & \left(\frac{1}{\lambda}+E\left(X_{1}\right)\right)\left(1-e^{-\lambda t}\right)-t e^{-\lambda t}+\sum_{i=1}^{2} p_{i} \frac{\lambda}{\lambda-\mu_{i}} t e^{-\lambda t}-\left(\sum_{i=1}^{2} p_{i} \frac{\lambda}{\left(\lambda-\mu_{i}\right)^{2}}+\right. \\
& \left.E\left(X_{1}\right) \sum_{i=1}^{2} p_{i} \frac{\lambda}{\lambda-\mu_{i}}\right)\left(e^{-\mu_{i} t}-e^{-\lambda t}\right)= \\
& \left.E\left(X_{1}\right) \sum_{i=1}^{2} p_{i} \frac{\lambda}{\lambda-\mu_{i}}\right)\left(e^{-\mu_{i} t}-e^{-\lambda t}\right) . \\
= & \left(\frac{1}{\lambda}+E\left(X_{1}\right)\right)\left(1-e^{-\lambda t}\right)+\sum_{i=1}^{2} p_{i} \frac{\mu_{i}}{\left(\lambda-\mu_{i}\right)^{2}} t e^{-\lambda t}-\left(\sum_{i=1}^{2} p_{i} \frac{\lambda}{\left(\lambda-\mu_{i}\right)^{2}}+\right. \\
& \\
& \\
& \\
&
\end{aligned}
$$

Por outro lado, fazendo-se de $I_{42}$ a segunda parcela da parte interna da integral da equação (5.6), temos

$$
\begin{aligned}
I_{42} & =\left(1+\frac{\lambda E\left(X_{1}\right)}{2}\right) \int_{0}^{t} \sum_{i=1}^{2} \frac{p_{i}}{\mu_{i}}\left(1-e^{-\mu_{i}(t-\tau)}\right) \lambda e^{-\lambda \tau} d \tau= \\
& =\left(1+\frac{\lambda E\left(X_{1}\right)}{2}\right) \lambda \sum_{i=1}^{2} \frac{p_{i}}{\mu_{i}}\left[\int_{0}^{t} e^{-\lambda \tau} d \tau-e^{-\mu_{i} t} \int_{0}^{t} e^{-\left(\lambda-\mu_{i}\right) \tau} d \tau\right]=
\end{aligned}
$$




$$
\begin{gathered}
=\left(1+\frac{\lambda E\left(X_{1}\right)}{2}\right) \lambda \sum_{i=1}^{2} \frac{p_{i}}{\mu_{i}}\left[\frac{1}{\lambda}\left(1-e^{-\lambda t}\right)-\left(1+\frac{\lambda E\left(X_{1}\right)}{2}\right) \lambda \sum_{i=1}^{2} \frac{p_{i}}{\mu_{i}} \frac{\lambda}{\lambda-\mu_{i}}\left(e^{-\mu_{i} t}-e^{-\lambda t}\right)\right]= \\
=\left(1+\frac{\lambda E\left(X_{1}\right)}{2}\right) E\left(X_{1}\right)\left(1-e^{-\lambda t}\right)-\left(1+\frac{\lambda E\left(X_{1}\right)}{2}\right) \sum_{i=1}^{2} \frac{p_{i}}{\mu_{i}} \frac{\lambda}{\lambda-\mu_{i}}\left(e^{-\mu_{i} t}-e^{-\lambda t}\right) .
\end{gathered}
$$

Fazendo-se de $I_{43}$ a terceira parcela da parte interna da integral da equação (5.6), temos

$$
\begin{aligned}
I_{43}= & -\left(1+\frac{\lambda E\left(X_{1}\right)}{2}\right) \sum_{i=1}^{2} p_{i} \int_{0}^{t}(t-\tau) e^{-\mu_{i}(t-\tau)} \lambda e^{-\lambda \tau}= \\
= & \left(1+\frac{\lambda E\left(X_{1}\right)}{2}\right) \sum_{i=1}^{2} p_{i} e^{-\mu_{i} t}\left[\int_{0}^{t} \tau e^{-\left(\lambda-\mu_{i}\right) \tau} d \tau-t \int_{0}^{t} e^{-\left(\lambda-\mu_{i}\right) r} d \tau\right]= \\
= & \left(1+\frac{\lambda E\left(X_{1}\right)}{2}\right) \sum_{i=1}^{2} p_{i} \frac{\lambda}{\left(\lambda-\mu_{i}\right)^{2}}\left(e^{-\mu_{i} t}-e^{-\lambda t}\right)-\left(1+\frac{\lambda E\left(X_{1}\right)}{2}\right) \sum_{i=1}^{2} p_{i} \frac{\lambda}{\lambda-\mu_{i}} t e^{-\lambda t} \\
& -\left(1+\frac{\lambda E\left(X_{1}\right)}{2}\right) \sum_{i=1}^{2} p_{i} \frac{\lambda}{\lambda-\mu_{i}}\left(e^{-\lambda t}-e^{-\lambda t}\right)= \\
= & \left(1+\frac{\lambda E\left(X_{1}\right)}{2}\right)\left[\left(\sum_{i=1}^{2} p_{i} \frac{\lambda}{\left(\lambda-\mu_{i}\right)^{2}}-\sum_{i=1}^{2} p_{i} \frac{\lambda}{\lambda-\mu_{i}}\right)\left(e^{-\mu_{i} t}-e^{-\lambda t}\right)-\sum_{i=1}^{2} p_{i} \frac{\lambda}{\lambda-\mu_{i}} t e^{-\lambda t}\right] .
\end{aligned}
$$

Assim, somando-se os três termos obtemos

$$
\begin{aligned}
I_{4}= & \left(\frac{1}{\lambda}+E\left(X_{1}\right)\right)\left(1-e^{-\lambda t}\right)+\sum_{i=1}^{2} p_{i} \frac{\mu_{i}}{\left(\lambda-\mu_{i}\right)^{2}} t e^{-\lambda t}-\left(\sum_{i=1}^{2} p_{i} \frac{\lambda}{\left(\lambda-\mu_{i}\right)^{2}}+\right. \\
& \left.E\left(X_{1}\right) \sum_{i=1}^{2} p_{i} \frac{\lambda}{\lambda-\mu_{i}}\right)\left(e^{-\mu_{i} t}-e^{-\lambda t}\right)+\left(1+\frac{\lambda E\left(X_{1}\right)}{2}\right) E\left(X_{1}\right)\left(1-e^{-\lambda t}\right)- \\
& \left(1+\frac{\lambda E\left(X_{1}\right)}{2}\right) \sum_{i=1}^{2} \frac{p_{i}}{\mu_{i}} \frac{\lambda}{\lambda-\mu_{i}}\left(e^{-\mu_{i} t}-e^{-\lambda t}\right)+\left(1+\frac{\lambda E\left(X_{1}\right)}{2}\right)\left[\left(\sum_{i=1}^{2} p_{i} \frac{\lambda}{\left(\lambda-\mu_{i}\right)^{2}}-\right.\right. \\
& \left.\left.\sum_{i=1}^{2} p_{i} \frac{\lambda}{\lambda-\mu_{i}}\right)\left(e^{-\mu_{i} t}-e^{-\lambda t}\right)-\sum_{i=1}^{2} p_{i} \frac{\lambda}{\lambda-\mu_{i}} t e^{-\lambda t}\right]= \\
= & \left(\frac{1}{\lambda}+2 E\left(X_{1}\right)+\left(\frac{1}{2}\right) \lambda E\left(X_{1}\right)^{2}\right)\left(1-e^{-\lambda t}\right)-t e^{-\lambda t}-\frac{\lambda E\left(X_{1}\right)}{2} \sum_{i=1}^{2} p_{i} \frac{\lambda}{\lambda-\mu_{i}} t e^{-\lambda t}
\end{aligned}
$$




$$
\begin{aligned}
& -\left[\sum_{i=1}^{2} p_{i} \frac{\lambda}{\left(\lambda-\mu_{i}\right)^{2}}+E\left(X_{1}\right) \sum_{i=1}^{2} p_{i} \frac{\lambda}{\lambda-\mu_{i}}+\sum_{i=1}^{2} \frac{p_{i}}{\mu_{i}} \frac{\lambda}{\lambda-\mu_{i}}+\frac{\lambda E\left(X_{1}\right)}{e} \sum_{i=1}^{2} \frac{p_{i}}{\mu_{i}} \frac{\lambda}{\lambda-\mu_{i}}\right. \\
& \left.-\sum_{i=1}^{2} p_{i} \frac{\lambda}{\left(\lambda-\mu_{i}\right)^{2}}-\sum_{i=1}^{2} p_{i} \frac{\lambda}{\left(\lambda-\mu_{i}\right)^{2}} \frac{\lambda E\left(X_{1}\right)^{2}}{2}\right]\left(e^{-\mu_{i} t}-e^{-\lambda t}\right)= \\
= & \left(\frac{1}{\lambda}+A\right)\left(1-e^{-\lambda t}\right)-t e^{-\lambda t}-\frac{\lambda E\left(X_{1}\right)}{2} \sum_{i=1}^{2} p_{i} \frac{\lambda}{\lambda-\mu_{i}} t e^{-\lambda t}-\left[E\left(X_{1}\right) \sum_{i=1}^{2} p_{i} \frac{\lambda}{\lambda-\mu_{i}}\right. \\
& \left.+\sum_{i=1}^{2} \frac{p_{i}}{\mu_{i}} \frac{\lambda}{\lambda-\mu_{i}}+\frac{\lambda E\left(X_{1}\right)}{2} \sum_{i=1}^{2} \frac{p_{i}}{\mu_{i}} \frac{\lambda}{\lambda-\mu_{i}}-\frac{\lambda E\left(X_{1}\right)^{2}}{2} \sum_{i=1}^{2} p_{i} \frac{\lambda}{\left(\lambda-\mu_{i}\right)^{2}}\right]\left(e^{-\mu_{i} t}-e^{-\lambda t}\right) .
\end{aligned}
$$

Logo,

$$
\begin{gathered}
E\left(Q * I\left(T+X_{1}<X_{2}\right) \mid 2\right)=\int_{0}^{\infty} I_{4} d P\left[X_{2} \leq t\right]= \\
=\left(\frac{1}{\lambda}+A\right)\left(1-\phi_{X_{2}}(\lambda)\right)+\left.\frac{d}{d s} \phi_{X_{2}}(s)\right|_{s=\lambda}+\frac{\lambda E\left(X_{1}\right)}{2} \sum_{i=1}^{2} p_{i} \frac{\lambda}{\lambda-\mu_{i}}\left(\left.\frac{d}{d s} \phi_{X_{2}}(s)\right|_{s=\lambda}\right) \\
-\left[E\left(X_{1}\right) \sum_{i=1}^{2} p_{i} \frac{\lambda}{\lambda-\mu_{i}}+\sum_{i=1}^{2} \frac{p_{i}}{\mu_{i}} \frac{\lambda}{\lambda-\mu_{i}}+\frac{\lambda E\left(X_{1}\right)}{2} \sum_{i=1}^{2} \frac{p_{i}}{\mu_{i}} \frac{\lambda}{\lambda-\mu_{i}}-\right. \\
\left.\frac{\lambda E\left(X_{1}\right)^{2}}{2} \sum_{i=1}^{2} \frac{p_{i}}{\mu_{i}} \frac{\lambda}{\lambda-\mu_{i}}\right]\left(\frac{d}{d s} \phi_{X_{2}}\left(\mu_{i}\right)-\frac{d}{d s} \phi_{X_{2}}(\lambda)\right) .
\end{gathered}
$$

O segundo termo da equação (5.6) é dado por

$$
\begin{aligned}
E\left(Q * I\left(T<X_{2}<T+X_{1}\right) \mid 2\right)= & \int_{0}^{\infty} \int_{0}^{t} \sum_{i=0}^{2} p_{i} e^{-\mu_{i}(t-\tau)}\left[t+\left(\frac{\lambda(t-\tau)}{2}+1\right) E\left(X_{2}\right)\right] \lambda . \\
& e^{-\lambda \tau} d \tau d P\left[X_{2} \leq t\right] .
\end{aligned}
$$

Integrando-se em $\tau$ e fazendo-se de $I_{5}$ igual ao termo interno da integral acima, temos

$$
I_{5}=\int_{0}^{t}\left[\sum_{i=0}^{2} p_{i} e^{-\mu_{i} t}\left(\left(1+\frac{\lambda E\left(X_{2}\right)}{2}\right) t e^{-\left(\lambda-\mu_{i}\right) \tau}+E\left(X_{2}\right) e^{-\left(\lambda-\mu_{i}\right) \tau}-\frac{\lambda E\left(X_{2}\right)}{2} \tau e^{-\left(\lambda-\mu_{i}\right) \tau}\right)\right] \lambda d \tau=
$$




$$
\begin{aligned}
= & \sum_{i=0}^{2} \lambda p_{i} e^{-\mu_{i} t}\left[\int_{0}^{t}\left(1+\frac{\lambda E\left(X_{2}\right)}{2}\right) t e^{-\left(\lambda-\mu_{i}\right) \tau} d \tau+E\left(X_{2}\right) \int_{0}^{t} e^{-\left(\lambda-\mu_{i}\right) \tau} d \tau-\right. \\
& \left.\frac{\lambda E\left(X_{2}\right)}{2} \int_{0}^{t} \tau e^{-\left(\lambda-\mu_{i}\right) \tau} d \tau\right]= \\
= & \sum_{i=0}^{2} \lambda p_{i} e^{-\mu_{i} t}\left[\left(1+\frac{\lambda E\left(X_{2}\right)}{2}\right) t \frac{1}{\lambda-\mu_{i}}\left(1-e^{-\left(\lambda-\mu_{i}\right) t}\right)+E\left(X_{2}\right) \frac{1}{\lambda-\mu_{i}}\left(1-e^{-\left(\lambda-\mu_{i}\right) t}\right)\right. \\
& \left.-\frac{\lambda E\left(X_{2}\right)}{2}\left(\frac{1}{\left(\lambda-\mu_{i}\right)^{2}}\left(1-e^{-\left(\lambda-\mu_{i}\right) t}\right)-\frac{t}{\lambda-\mu_{i}} e^{-\left(\lambda-\mu_{i}\right) t}\right)\right]= \\
= & \sum_{i=0}^{2} p_{i} \frac{\lambda}{\lambda-\mu_{i}}\left\{\left[\left(1+\frac{\lambda E\left(X_{2}\right)}{2}\right) t-\frac{\lambda E\left(X_{2}\right)}{2\left(\lambda-\mu_{i}\right)}+E\left(X_{2}\right)\right]\left(e^{-\mu_{i} t}-e^{-\lambda t}\right)+\frac{\lambda E\left(X_{2}\right)}{2} t e^{-\lambda t}\right\} .
\end{aligned}
$$

Logo,

$$
E\left(Q * I\left(T<X_{2}<T+X_{1}\right) \mid 2\right)=\int_{0}^{\infty} I_{5} d P\left[X_{2} \leq t\right]
$$

Substituindo-se o valor de $I_{5}$ na equação acima e integrando-o em relação a $P\left[X_{2} \leq t\right]$, obtemos

$$
\begin{gathered}
E\left(Q * I\left(T<X_{2}<T+X_{1}\right) \mid 2\right)=\sum_{i=0}^{2} p_{i} \frac{\lambda}{\lambda-\mu_{i}} \frac{\lambda E\left(X_{2}\right)}{2}\left(-\left.\frac{d}{d s} \phi_{X_{2}}(s)\right|_{s=\lambda}\right)+ \\
\sum_{i=0}^{2} p_{i} \frac{\lambda}{\lambda-\mu_{i}}\left[\left(1+\frac{\lambda E\left(X_{2}\right)}{2}\right)\left(-\left.\frac{d}{d s} \phi_{X_{2}}(s)\right|_{s=\mu_{i}}\right)+\left.\frac{d}{d s} \phi_{X_{2}}(s)\right|_{s=\lambda}\right]+\sum_{i=0}^{2} p_{i} \frac{\lambda}{\lambda-\mu_{i}}\left(E\left(X_{2}\right)-\right. \\
\frac{\lambda E\left(X_{2}\right)}{2\left(\lambda-\mu_{i}\right)}\left(\phi_{X_{2}}\left(\mu_{i}\right)-\phi_{X_{2}}(\lambda)\right) .
\end{gathered}
$$

Assim, somando-se os resultados dos dois termos da Equação 5.6, obtemos

$$
\begin{aligned}
E(Q \mid 2)= & E\left(X_{2}\right) \phi_{X_{2}}(\lambda)+\left(\frac{1}{\lambda}+A\right)\left(1-\phi_{X_{2}}(\lambda)\right)+\sum_{i=0}^{2} p_{i} \frac{\lambda}{\lambda-\mu_{i}}\left[\{ \phi _ { X _ { 2 } } ( \mu _ { i } ) - \phi _ { X _ { 2 } } ( \lambda ) \} \left[E\left(X_{2}\right)\right.\right. \\
& \left.\left.-E\left(X_{1}\right)-\left(1+\frac{\lambda E\left(X_{2}\right)}{2}\right) \frac{1}{\mu_{i}}-\frac{B}{\lambda-\mu_{i}}\right]-\left.B \frac{d}{d s} \phi_{X_{2}}(s)\right|_{s=\mu_{i}}\right] .
\end{aligned}
$$

Aqui a prova da igualdade da equação (5.6). 


\subsection{Resultados Numéricos}

Apresentamos nesta seção os resultados numéricos e a análise do tempo médio de permanência do cliente esperto para os sistemas de filas: $\quad M /((M+M) / 1)^{2}, \quad M /((M+$ $\left.\left.E_{2}\right) / 1\right)^{2}, M /\left(\left(M+E_{3}\right) / 1\right)^{2}$ e $M /\left(\left(M+H_{2}\right) / 1\right)^{2}$. Fixamos para os sistema de filas acima o tempo médio de serviço do primeiro servidor em 1 e assumimos os níveis de ocupações $\rho=\frac{\lambda}{\mu_{1}+\mu_{2}}$ variando de 0,1 a 0,9 . Consideremos os seguintes valores para os tempos médios de serviço do segundo servidor: $E\left(X_{2}\right)=10 ; 5 ; 2,5 ; 1 ; 0,5$ e 0,1 , onde $E[T C C S \mid 1]$ : o tempo médio de permanência do cliente esperto sob a estratégia de unir-se à fila mais curta. Em cada tabela temos os tempos médios sob as duas estratégias.

Na Tabela 5.1, para o sistema de filas $M /((M+M) / 1)^{2}$ verificamos que para os valores de $\rho>0,6$ e $E\left(X_{2}\right)=10$, temos que o tempo médio de permanência do cliente esperto sob a estratégia de observar as filas paralelas antes de unir-se a uma delas é melhor do que a estratégia de unir-se à fila mais curta. Percebemos que se $\frac{E X_{1}}{10}<E X_{2}<10 E X_{1}$, então a estratégia de unir-se à fila mais curta é melhor.

Nas Tabelas 5.2 e 5.3, apresentam-se o tempo médio de permanência do cliente esperto nos sistemas $M /\left(\left(M+E_{2}\right) / 1\right)^{2}$ e $M /\left(\left(M+E_{3}\right) / 1\right)^{2}$. Verificamos que para $\rho>0,4 \mathrm{e}$ $E\left(X_{2}\right)=10$, temos que a estratégia de observar as filas paralelas antes de unir-se a uma delas é melhor do que unir-se à fila mais curta. Concluímos da mesma forma que se $\frac{E X_{1}}{10}<E X_{2}<10 E X_{1}$, então a estratégia de unir-se à fila mais curta apresenta uma melhor performance.

Na Tabela 5.4, apresenta-se o tempo médio de permanência do cliente esperto no sistema $M /\left(\left(M+H_{2}\right) / 1\right)^{2}, C_{2}{ }^{2}=9$. Lembramos que a distribuição $H_{2}$ tem médias balanceadas. Verificamos que para qualquer tempo médio de serviço do segundo servidor, sob a estratégia de observar as filas paralelas antes de unir-se a uma delas, apresenta o tempo médio de permanência maior do que na estratégia de unir-se à fila mais curta, exceto quando tomamos $E X_{2}=0,1$ e $\rho>0,5$. 
Tabela 5.1 - O Tempo Médio de espera do cliente esperto no Sistema $M /((M+M) / 1)^{2}$

\begin{tabular}{|c|c|c|c|c|c|c|}
\hline$\rho$ & $E X_{2}=10$ & 5 & 2,5 & 1 & 0,5 & 0,1 \\
\hline 0,1 & 15,631 & 14,316 & 12,111 & 9,681 & 4,724 & 1,563 \\
0,2 & 9,752 & 8,799 & 7,538 & 6,006 & 2,999 & 0,975 \\
0,3 & 7,631 & 6,849 & 5,946 & 4,765 & 2,413 & 0,763 \\
0,4 & 6,551 & 5,865 & 5,145 & 4,150 & 2,122 & 0,655 \\
0,5 & 5,912 & 5,287 & 4,674 & 3,793 & 1,952 & 0,591 \\
0,6 & 5,505 & 4,919 & 4,375 & 3,568 & 1,846 & 0,551 \\
0,7 & 5,233 & 4,674 & 4,177 & 3,420 & 1,776 & 0,523 \\
0,8 & 5,048 & 4,507 & 4,043 & 3,321 & 1,731 & 0,505 \\
0,9 & 4,922 & 4,393 & 3,953 & 3,256 & 1,701 & 0,492 \\
\hline$E[T C S \mid 1]$ & 5,5 & 3,00 & 1,75 & 1,0 & 0,75 & 0,55 \\
\hline
\end{tabular}

Tabela 5.2 - O Tempo Médio de espera do cliente esperto no Sistema $M /\left(\left(M+E_{2}\right) / 1\right)^{2}$

\begin{tabular}{|c|c|c|c|c|c|c|}
\hline$\rho$ & $E X_{2}=10$ & 5 & 2,5 & 1 & 0,5 & 0,1 \\
\hline 0,1 & 15,643 & 13,062 & 10,351 & 6,944 & 4,731 & 1,563 \\
0,2 & 9,537 & 8,261 & 6,610 & 4,418 & 3,012 & 0,975 \\
0,3 & 7,293 & 6,521 & 5,322 & 3,577 & 2,430 & 0,762 \\
0,4 & 6,143 & 5,623 & 4,675 & 3,165 & 2,141 & 0,653 \\
0,5 & 5,462 & 5,087 & 4,296 & 2,931 & 1,974 & 0,588 \\
0,6 & 5,025 & 4,742 & 4,058 & 2,786 & 1,870 & 0,547 \\
0,7 & 4,730 & 4,512 & 3,903 & 2,695 & 1,802 & 0,519 \\
0,8 & 4,527 & 4,356 & 3,802 & 2,637 & 1,759 & 0,499 \\
0,9 & 4,384 & 4,251 & 3,739 & 2,603 & 1,731 & 0,486 \\
\hline$E[T C S \mid 1]$ & 5,5 & 3,00 & 1,75 & 1,0 & 0,75 & 0,55 \\
\hline
\end{tabular}

Tabela 5.3 - O Tempo Médio de espera do cliente esperto no Sistema $M /\left(\left(M+E_{3}\right) / 1\right)^{2}$

\begin{tabular}{|c|c|c|c|c|c|c|}
\hline$\rho$ & $E\left(X_{2}\right)=10$ & 5 & 2,5 & 1 & 0,5 & 0,1 \\
\hline 0,1 & 15,646 & 13,096 & 10,372 & 6,951 & 4,734 & 1,563 \\
0,2 & 9,444 & 8,272 & 6,632 & 4,429 & 3,016 & 0,974 \\
0,3 & 7,151 & 6,507 & 5,348 & 3,592 & 2,435 & 0,761 \\
0,4 & 5,979 & 5,590 & 4,700 & 3,184 & 2,147 & 0,652 \\
0,5 & 5,286 & 5,040 & 4,321 & 2,952 & 1,981 & 0,587 \\
0,6 & 4,841 & 4,685 & 4,082 & 2,810 & 1,878 & 0,545 \\
0,7 & 4,541 & 4,448 & 3,928 & 2,722 & 1,811 & 0,517 \\
0,8 & 4,333 & 4,286 & 3,829 & 2,667 & 1,768 & 0,497 \\
0,9 & 4,185 & 4,175 & 3,765 & 2,635 & 1,741 & 0,484 \\
\hline$E[T C S \mid 1]$ & 5,5 & 3,00 & 1,75 & 1,0 & 0,75 & 0,55 \\
\hline
\end{tabular}


Tabela 5.4 - O Tempo Médio de espera do cliente esperto

\begin{tabular}{|c|c|c|c|c|c|c|}
\hline \multicolumn{7}{|c|}{ no Sistema $M /\left(\left(M+H_{2}\right) / 1\right)^{2} C_{2}^{2}=9$} \\
\hline$\rho$ & $E\left(X_{2}\right)=10$ & 5 & 2,5 & 1 & 0,5 & 0,1 \\
0,1 & 15,617 & 12,715 & 10,057 & 6,798 & 4,655 & 1,554 \\
0,3 & 10,095 & 8,192 & 6,369 & 4,237 & 2,904 & 0,964 \\
0,4 & 8,091 & 6,620 & 5,129 & 3,375 & 2,301 & 0,752 \\
0,5 & 7,060 & 5,826 & 4,514 & 2,947 & 1,995 & 0,645 \\
0,6 & 6,450 & 5,358 & 4,156 & 2,695 & 1,813 & 0,583 \\
0,7 & 6,061 & 5,062 & 3,930 & 2,534 & 1,694 & 0,544 \\
0,8 & 5,804 & 4,867 & 3,781 & 2,426 & 1,613 & 0,518 \\
0,9 & 5,633 & 4,737 & 3,681 & 2,351 & 1,557 & 0,502 \\
\hline$E[T C S \mid 1]$ & 5,521 & 4,652 & 3,616 & 2,300 & 1,516 & 0,491 \\
\hline
\end{tabular}

\subsection{Considerações Finais}

Neste capítulo analisamos o tempo médio de permanência do cliente esperto para duas estratégias distintas e comparamos os tempos médios entre as duas. No próximo capítulo apresentamos uma proposta para futuros trabalhos, no intuito de complementar esta dissertação. 



\section{Capítulo 6}

\section{Comparação Numérica dos Resultados}

\subsection{Introdução}

Neste capítulo apresentamos primeiramente o sistema de filas $M / M / 2$, com suas características e algumas medidas de performance. De acordo com estas medidas, realizamos uma comparação entre os modelos dos sistemas apresentados.

A fila $M / M / 2$ tem as seguintes características: dois servidores em paralelo e uma única fila; cada cliente é atendido por apenas um dos servidores com uma disciplina FCFS os clientes são servidos na ordem de chegada; os dois servidores atendem os clientes com tempo de serviço distribuído exponencialmente i.i.d.; a sala de espera é ilimitada; os tempos entre as chegadas são variáveis aleatórias exponenciais i.i.d. Os tempos entre as chegadas e os de serviço de cada um dos servidores são independentes. (Veja a Figura 6.1.)

Indicamos a seguir algumas medidas de performance de interesse do sistema de filas $M / M / 2$, que são determinadas exatamente pelas seguintes equações, onde $\rho$ é a taxa de ocupação do sistema. Para $\rho<1$ :

$$
\rho=\frac{\lambda}{2 \mu} .
$$


Número esperado de clientes no sistema:

$$
L=\sum_{n=0}^{\infty} n \pi_{n}=\frac{2 \rho}{1-\rho^{2}}=\frac{4 \lambda \mu}{4 \mu^{2}-\lambda^{2}} .
$$

Tempo médio de permanência do cliente no sistema:

$$
W=\frac{2 \rho}{\lambda\left(1-\rho^{2}\right)}=\frac{4 \mu}{4 \mu^{2}-\lambda^{2}}
$$

O tempo médio de permanência e o número esperado de clientes no sistema, citados acima, demonstra que, conhecendo-se uma dessas medidas de performance obtemos a outra e vice-versa, aplicando-se a fórmula de Little, que é dada por:

$$
L=\lambda W
$$

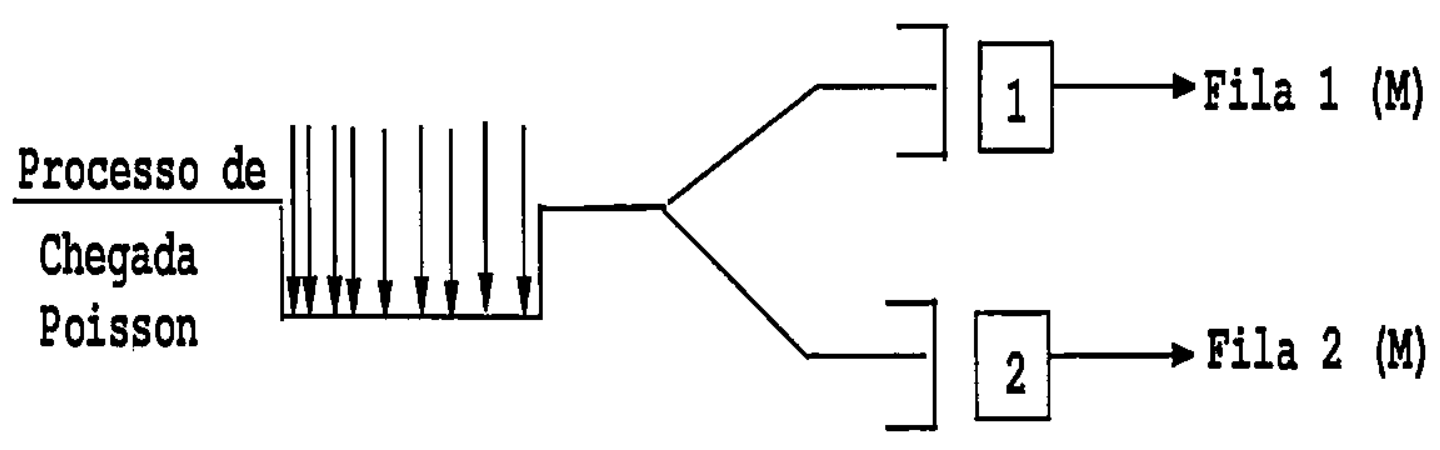

Figura 6.1: Uma das representação do modelo $M / M / 2$

\subsection{Resultados Encontrados}

Ressaltamos alguns procedimentos importantes que utilizamos, antes de qualquer comparação entre os modelos do sistema de filas em paralelo, considerando-se os parâmetros pré-estabelecidos, conforme a descriçāo a seguir. Utilizamos vários valores para as taxas de $\lambda, \mu_{1}$ e $\mu_{2}$, calculamos o número esperado de clientes e o tempo médio do cliente no sistema. 
Apresentamos a seguir os resultados encontrados para os sistemas de filas: $M / M / 2$ e os modelos de filas em paralelo. Nas tabelas apresentadas abaixo, consideramos para o modelo de fila da Tabela 6.1 as taxas $\left(\rho, \lambda, \mu_{1}, \mu_{2}\right)=(0,4167 ; 5,0000 ; 6,0000 ; 6,0000)$, $(0,8333 ; 10,0000 ; 6,0000 ; 6,0000)$ e demais tabelas acrescentamos as taxas $\left(\rho, \lambda, \mu_{1}, \mu_{2}\right)=$ $(0,4167 ; 5,0000 ; 1,0000 ; 0,0300),(0,4167 ; 5,0000 ; 1,0000 ; 11,000),(0,9000 ; 0,9270$; $1,0000 ; 0,0300)$ e $(0,9500 ; 0,9785 ; 1,0000 ; 0,03000)$, de ocupação, de chegada, as de serviços no sistema, respectivamente.

Tabela 6.1 - Número Esperado e Tempo Médio de permanência do cliente no sistema de fila $M / M / 2$

\begin{tabular}{|c|c|c|c|c|c|}
\hline$\rho$ & $\lambda$ & $\mu_{\mathbf{1}}$ & $\mu_{2}$ & L & W \\
\hline $\mathbf{0 , 4 1 6 7}$ & $\mathbf{5 , 0 0 0 0}$ & 6,0000 & 6,0000 & $\mathbf{1 , 0 0 8 4}$ & $\mathbf{0 , 2 0 1 7}$ \\
\hline $\mathbf{0 , 8 3 3 3}$ & 10,0000 & 6,0000 & 6,0000 & $\mathbf{5 , 4 5 4 5}$ & $\mathbf{0 , 5 4 5 4}$ \\
\hline
\end{tabular}

A Tabela 6.1 mostra os resultados de $L$ e $W$ para o sistema de fila única $M / M / 2$, com taxas $\rho=0,4167, \lambda=5, \mu_{1}=\mu_{2}=6$ e $\rho=0,833, \lambda=10, \mu_{1}=\mu_{2}=6$ de ocupações, chegadas e serviços, respectivamente.

Tabela 6.2 - Número Esperado e Tempo Médio de permanência do cliente no sistema de fila $M /(M / 1)^{2}$ - Haight(57)

\begin{tabular}{|c|c|c|c|c|c|}
\hline$\rho$ & $\lambda$ & $\mu_{1}$ & $\mu_{\mathbf{2}}$ & $\mathrm{L}$ & $\mathbf{W}$ \\
\hline $\mathbf{0 , 4 1 6 7}$ & 0,4292 & 1,0000 & 0,0300 & $\mathbf{2 , 5 0 9 9}$ & $\mathbf{5 , 8 4 7 9}$ \\
\hline $\mathbf{0 , 4 1 6 7}$ & 5,0000 & $\mathbf{6 , 0 0 0 0}$ & 6,0000 & $\mathbf{1 , 0 6 7 5}$ & $\mathbf{0 , 2 1 3 5}$ \\
\hline $\mathbf{0 , 4 1 6 7}$ & 5,0000 & 1,0000 & 11,0000 & $\mathbf{2 , 4 2 2 5}$ & $\mathbf{0 , 4 8 4 5}$ \\
\hline $\mathbf{0 , 8 3 3 3}$ & 10,0000 & 6,0000 & 6,0000 & $\mathbf{3 , 7 8 5 9}$ & $\mathbf{0 , 3 7 8 6}$ \\
\hline $\mathbf{0 , 9 0 0 0}$ & 0,9270 & 1,0000 & 0,0300 & $\mathbf{5 , 5 1 2 2}$ & $\mathbf{6 , 1 2 4 7}$ \\
\hline $\mathbf{0 , 9 5 0 0}$ & 0,9785 & 1,0000 & 0,0300 & $\mathbf{5 , 5 4 9 5}$ & $\mathbf{5 , 8 4 1 6}$ \\
\hline
\end{tabular}

A Tabela 6.2 mostra os resultados de $L$ e $W$ para o modelo do sistema de fila $M /(M / 1)^{2}$, que descrevemos no capítulo 2 em relação ao artigo de Haight(57). Comparamos este modelo com o sistema de fila única. Verificamos que em $\rho=0,4167$ a fila única apresenta um melhor desempenho, porém em $\rho=0,8333$ o sistema de fila em paralelo passa a ter um melhor desempenho do que a fila única. 
Tabela 6.3 - Número Esperado e Tempo Médio de permanência do cliente no sistema de fila $M /(M / 1)^{2}$ Hlynka, Stanford, Poon e Wang (94)

\begin{tabular}{|c|c|c|c|c|c|}
\hline$\rho$ & $\lambda$ & $\mu_{1}$ & $\mu_{2}$ & $\mathrm{~L}$ & $\mathrm{~W}$ \\
\hline $\mathbf{0 , 4 1 6 7}$ & 0,4292 & 1,0000 & 0,0300 & $\mathbf{4 , 4 2 3 7}$ & $\mathbf{1 0 , 3 0 6 9}$ \\
\hline $\mathbf{0 , 4 1 6 7}$ & 5,0000 & 6,0000 & 6,0000 & $\mathbf{4 , 4 2 3 4}$ & $\mathbf{0 , 8 8 4 7}$ \\
\hline $\mathbf{0 , 8 3 3 3}$ & 10,0000 & 6,0000 & 6,0000 & $\mathbf{6 , 1 9 5 4}$ & $\mathbf{0 , 6 1 9 5}$ \\
\hline $\mathbf{0 , 9 0 0 0}$ & 0,9270 & 1,0000 & 0,0300 & $\mathbf{6 , 7 5 2 5}$ & $\mathbf{6 , 9 0 0 9}$ \\
\hline
\end{tabular}

A Tabela 6.3 mostra os resultados do número esperado de clientes e o tempo médio do cliente no sistema de filas em paralelo, onde o cliente esperto utiliza a estratégia de observar as filas antes de juntar-se a elas. Verificamos que as medidas de performance nesta tabela são maiores do que o modelo proposto por Haight(57)(veja a Tabela 6.2). De fato, era de se esperar, pois o modelo analisado por Hlynka, Stanford, Poon e Wang (94), considera que o cliente esperto, ao entrar no sistema, o encontra vazio. Daí ele observa um ou mais novos clientes se juntarem ao sistema e tomarem seus lugares na fila à sua frente. Então o cliente esperto escolhe a fila na qual ocorre o primeiro fim de serviço. No sistema de fila proposto por Haight(57), os clientes que chegam ao sistema somente observam os comprimentos entre as filas e juntam-se à mais curta. É importante salientar que, se uma das taxas de serviço for pequena e a outra grande, mostramos no capítulo 4 que o cliente esperto tem um desempenho melhor quando observa as filas antes de juntar-se a uma delas.

Tabela 6.4 - Número Esperado e Tempo Médio de permanência do cliente no sistema de fila $M /(M / 1)^{2}$ Stanford e Hlynka (96)

\begin{tabular}{|c|c|c|c|c|c|}
\hline$\rho$ & $\lambda$ & $\mu_{1}$ & $\mu_{2}$ & $\mathrm{~L}$ & $\mathrm{~W}$ \\
\hline $\mathbf{0 , 4 1 6 7}$ & $0, \overline{4292}$ & 1,0000 & 0,03000 & $\mathbf{0 , 5 4 0 7}$ & $\mathbf{1 , 2 5 9 7}$ \\
\hline $\mathbf{0 , 4 1 6 7}$ & 5,000 & $\mathbf{6}, 0000$ & 6,0000 & $\mathbf{2 9 , 0 4 9 2}$ & $\mathbf{5 , 8 0 9 8}$ \\
\hline $\mathbf{0 , 8 3 3 3}$ & 10,0000 & $\mathbf{6 , 0 0 0 0}$ & $\mathbf{6 , 0 0 0 0}$ & $\mathbf{4 4 , 2 1 6 0}$ & $\mathbf{4 , 4 2 1 6}$ \\
\hline $\mathbf{0 , 9 0 0 0}$ & 0,9270 & 1,0000 & 0,0300 & $\mathbf{1 , 3 0 0 7}$ & $\mathbf{1 , 4 0 3 1}$ \\
\hline $\mathbf{0 , 9 5 0 0}$ & 0,9785 & 1,0000 & 0,0300 & $\mathbf{2 , 6 9 8 4}$ & $\mathbf{2 , 8 4 4 0 4}$ \\
\hline
\end{tabular}

A Tabela 6.4 mostra que as medidas de desempenho, o número esperado de clientes 
e o tempo médio de permanência do cliente no sistema estudado por Stanford e Hlynka (96) apresenta performance melhor do que o modelo proposto por Haight(57).

Nos modelos das Tabelas 6.3 e 6.4, dos capítulos 4 e 5, foi analisado o número médio de permanência do cliente esperto no sistema. Constatamos que para certos valores das taxas de serviços e taxa de ocupação, o tempo médio de permanência do cliente esperto nestes modelos é melhor do que unir-se à fila mais curta. De fato, quando o cliente esperto espera e junta-se à fila que produz o primeiro fim de serviço, o desempenho é melhor. 



\section{Capítulo 7}

\section{Conclusões Finais e Propostas Futuras}

Essa dissertação trata da obtenção de medidas de desempenho de filas em paralelo, através de soluçōes analíticas exatas. É tratado a comparação com algumas medidas de desempenho do sistema de fila única, a $M / M / 2$.

Foram abordados diferentes critérios de como juntar-se à fila, bem como diferentes distribuições de tempo de serviço. Analisamos primeiramente o artigo de Haight(58), onde em um sistema $M /(M / 1)^{2}$ o cliente que acaba de chegar ao sistema segue o critério tradicional de juntar-se à fila mais curta, com opção pela fila 1 quando as duas apresentam o mesmo comprimento. São apresentadas a distribuição de equilíbrio conjunta e as marginais, da variável bidimensional que conta o número de clientes nas filas 1 e 2 e o tempo médio de espera na fila.

Apresentamos o modelo proposto por Whitt(89), cujo critério de juntar-se à fila mais curta nem sempre é a melhor escolha. O modelo definido é um sistema de fila $M /(G / 1)^{2}$, onde o tempo de serviço é tal que o comprimento de serviço é zero, com probabilidade muito alta $1-\epsilon$, ou $n$ com probabilidade muita baixa ( $\epsilon$, que é muito pequeno). O critério para juntar-se à fila é o seguinte: quando as duas têm o mesmo comprimento, a escolha é aleatória; quando a diferença entre os comprimentos das filas é 1, escolhe-se a fila menor; e quando a diferença entre elas é de pelo menos 2, escolhe-se a fila maior. Com este tempo de serviço e este critério de escolha da fila, indicamos como é calculado o tempo médio de 
espera na fila.

Finalmente são apresentados os modelos propostos por Hlynka et al.(94) e Stanford e Hlynka(96), que são os sistemas de filas $G /\left(\left(M_{1}+M_{2}\right) / 1\right)^{2}$ e $M /((M+G) / 1)^{2}$, respectivamente, onde o cliente esperto utiliza o critério de esperar e juntar-se à fila que produz o primeiro fim de serviço. Para este critério são apresentados os cálculos do tempo médio de permanência do cliente esperto no sistema em cada modelo.

Constatamos que ocorreram diferenças entre os resultados numéricos obtidos através dos artigos de Hlynka et al.(94) e de Haight(57) devido à abordagem feita em cada modelo. No primeiro modelo eles consideraram que o cliente esperto quando chega no sistema o encontra vazio, enquanto que no segundo modelo os clientes que chegam no sistema utilizam o critério de juntar-se à fila mais curta.

Como podemos ver pelos resultados apresentados nos capítulos 4 e 5 , para certos parâmetros concluímos que o cliente esperto apresenta um desempenho melhor, quando este observa as filas antes de juntar-se a uma delas.

\subsection{Proposta Futura}

Apresentamos a seguir uma proposta que poderá ser implementada a esta nossa pesquisa, dando sustentação aos resultados obtidos até então, ou apontando nova alternativa.

- Verificar as medidas de desempenho de um sistema de fila única, com solução analítica exata, porém com taxas de serviços diferentes. 


\section{Apêndice A}

\section{Algoritmo do Programa}

Passo 1: fixamos os inteiros positivos, $\mathrm{n}, \lambda, \mu, \mu^{\prime}$ e calculamos a equação geratriz de probabilidade que é dada por $f(x, y)$;

Passo 2: calculamos as derivadas parciais para os $n$ clientes no sistema, atribuindo os seguintes valores aos pares $\left(s, s^{\prime}\right):(0,0),(1,0),(0,1)$ e $(1,1)$, obtendo um conjunto de equações globais de balanços;

Passo 3: resolvemos o sistema de equação obtendo a distribuição de equilíbrio.

Clear[n]

$\mathrm{n}=\mathrm{n}$,

Clear $\left[\lambda, \mu, \mu^{\prime}\right]$

$\lambda=5, \mu=6, \mu^{\prime}=8$;

Função Geradora de Probabilidade do Sistema

Clear[f]

$$
\begin{aligned}
f[x, y]= & \mu * \operatorname{Sum}\left[p[0, j] *\left(y^{j}\right), j, 0, n\right]+\mu^{\prime} * \operatorname{Sum}\left[p[i, 0] *\left(x^{i}\right), i, 0, n\right]-\left(\lambda+\mu+\mu^{\prime}\right) * \\
& \operatorname{Sum}\left[p[i, j] *\left(x^{i}\right) *\left(y^{j}\right), i, 0, n, j, 0, n\right]+\mu^{\prime} * \operatorname{Sum}\left[p[i, j] *\left(x^{i}\right) *\left(y^{j-1}\right), i, 0, n, j, 1, n\right]+ \\
& \mu * \operatorname{Sum}\left[p[i, j] *\left(x^{i-1}\right) *\left(y^{j}\right), i, 1, n, j, 0, n\right]+\lambda * \operatorname{Sum}\left[\left(x^{i}\right) *(\operatorname{Sum}[p[i-1, j] *\right. \\
& \left.\left.\left.\left(y^{j}\right), j, i-1, n\right]\right), i, 1, n+1\right]+\lambda *(y) * \operatorname{Sum}\left[\left(x^{i}\right) * \operatorname{Sum}\left[p[i, j] *\left(y^{j}\right), j, 0, i-1\right], i, 1, n\right]
\end{aligned}
$$

Função Derivada do Sistema

Clear $[\mathrm{h}]$ 
For $[i=0, i \leq n, i++, F \operatorname{or}[j=0, j \leq n, j++, h[x, y, i, j]=D[D[f[x, y], x, i], y, j]]]$

Variáveis do Sistema

Clear[vetvar]

vetvar $=; \operatorname{For}[i=0, i \leq n, i++, \operatorname{For}[j=0, j \leq n, j++, \operatorname{vetvar}=$ Union $[$ vetvar,$p[i, j]]]]$

\section{Equaçōes do Sistema}

Clear[veteq]

$$
\begin{gathered}
\text { veteq }=\operatorname{Sum}[p[i, j], i, 0, n, j, 0, n]==1 ; \text { For }[i=0, i \leq n-1, i++, \\
\text { veteq }=\text { Union }[\text { vet }, \text { Simplify }[h[0,0, i, 0]]==0] ; \\
\text { veteq }=\text { Union }[\text { vet }, \text { Simplify }[h[1,0, i, 0]]==0] ; \\
\text { veteq }=\text { Union }[\text { vetq, Simplify }[h[0,1, i, 0]]==0] ; \\
\text { If }[n \leq 2, \text { veteq }=\text { Union }[\text { vetq, Simplify }[h[1,1, i, 0]]==0]] ;
\end{gathered}
$$

For $\quad[j=0, j \leq n-1, j++$, veteq $=$ Union $[$ veteq, Simplify $[h[0,0,0, j]]==0]$

$$
\begin{aligned}
& \text { vete }=\text { Union }[\text { veteq, Simplify }[h[1,0,0, j]]==0] \\
& \text { veteq }=\text { Union }[\text { veteq, Simplify }[h[0,1,0, j]]==0] \\
& \operatorname{If}[n \leq 2, \text { veteq }=\text { Union }[\text { veteq, Simplify }[h[1,1,0, j]]==0]]] ;
\end{aligned}
$$

For $\quad[i=0, i \leq n, i++, F o r[j=0, j \leq n, j++, I f[(i+j) \leq n$,

$$
\text { veteq }=\text { Union }[\text { veteq, Simplify }[h[1,0, i, j]]==0]]]
$$

For $\quad[i=0, i \leq n, i++$, For $[j=0, j \leq n, j++, \operatorname{If}[(i+j) \leq(2 * n-4)$,

$$
\text { veteq }=\text { Union }[\text { veteq, Simplify }[h[0,0, i, j]]==0]]]]
$$

\section{Solução das Variáveis do Sistema}

Solve[veteq, vetvar]

Solução da distribuição do Sistema

Clear[vetvard]

vetvard $=\{\}$; 
For $[i=0, i \leq n, i++$, vetvard $=[\operatorname{vetvard}, x[i]]]$

$$
\begin{gathered}
\text { veteqd }=\text { Union }\left[\text { veteqd, } x[j]==(1 / \rho) *(x[j+1]-p[0, j])+\left(1 / \rho^{\prime}\right) *(x[j+1]\right. \\
-p[j, 0])]] ;
\end{gathered}
$$

Solve[vetvard,veteqd] 
, 


\section{Referências Bibliográficas}

CIINLAR, E. Introduction to Stochastic Process. Englewood Cliffs, New Jersey, Prentice-Hall, 1975.

HAIGHT, F. A. Two Queues in Parallel. Biometrika, vol. 45, pp. 401-410, 1958.

HLYNKA, M.; STANFORD, D. A.; POON, W. H. and WANG, T.

Observing Queues before Joining. Operations Research, vol. 42(2), pp. 356$371,1961$.

FLATTO,L., and MCKEAN,H.P. Two Queues in Parallel. Comm. Pure and Appl. Math. vol. 30, pp. 255-263, 1977.

KINGMAN, J. F. C. Two Similar Queues in Parallel. it Ann Math. Stat. vol. 2, pp. 1314-1323, 1961.

HLYNKA, M.; STANFORD, D. A.; POON, W. H. and WANG, T. Observing Queues before Joining. Operations Research, vol. 42(2), pp. 356-371, 1961.

LEHTONEN, T. On the Optimality of the Shortest Line Discipline. Chapter IV in Stochastic Comparisons for many Server Queues Ph.D dissertation, The Helsinki School of Economics.

STANFORD, D. A. and HLYNKA, M. Observing General Service Queues before Joining. Operations Research Letters, vol. 18 (5), pp. 237-245, 1996. 
WEBER, R. W. On the Optimal Assignment of Customers to Parallel Servers. J. Appl. Prob., vol. 15, pp. 406-413, 1978.

WHITT, W. Deciding which Queue to Join: Some Counterexamples. Operations Research, vol. 34, pp. 55-62, 1986.

WINSTON, W. Optimality of the Shortest Line Discipline. J. Appl. Prob., vol. 14, pp. 181-189, 1977.

WOLFF, R. W. Stochastic Modelling and the Theory of Queues. Englewoods Cliffs, New Jersey, Prentice-Hall, 1988. 